Supporting Information

\title{
Blended Assemblies of Amphiphilic Random and Block Copolymers for Tunable Encapsulation and Release of Hydrophobic Guest Molecules
}

Ziwen Jiang, ${ }^{\dagger, \S}$ Hongxu Liu, ${ }^{\dagger, \S}$ Huan He ${ }^{\dagger}$ Alexander E. Ribbe,,${ }^{\ddagger}$ S. Thayumanavan ${ }^{*, \dagger}$

${ }^{\dagger}$ Department of Chemistry, ‡Department of Polymer Science and Engineering, University of Massachusetts Amherst, Amherst, Massachusetts 01003, United States

$\S Z$.J. and H.L. equally contributed to this work.

*Email: thai@chem.umass.edu (S.T.) 


\section{Table of Contents}

\section{Supplementary figures and table}

Figure S1. HAADF-STEM images and the corresponding EDS analysis of the R73-Br-B73-Fe co-assemblies.

Figure S2. SEM image of R73-B73 co-assemblies.

Figure S3. FRET evaluation of the polymeric co-assemblies.

Figure S4. Dry-state TEM images of (co-)assemblies between $\mathbf{R 7 3 - B r}$ and $\mathbf{B 7 3 - F e}$ at different mass ratios.

Figure S5. Debye plots from static light scattering for (a) standard PEG and (b) B73 self-assemblies in water.

Figure S6. Debye plots from static light scattering for R37-B73 assemblies at different mass ratios.

Figure S7. Debye plots from static light scattering for R55-B73 assemblies at different mass ratios.

Figure S8. Debye plots from static light scattering for R73-B73 assemblies at different mass ratios.

Figure S9. Debye plots from static light scattering for R91-B73 assemblies at different mass ratios.

Figure S10. Aggregation number of each (co-)assemblies at different mass ratios.

Table S1. Loading capacity and encapsulation efficiency of Nile red in R73 and B73 with optimized protocols.

Figure S11. Standard calibration curve for Nile red, Sudan red G, Sudan black B, and Oil red O.

Figure S12. Critical aggregation concentration (CAC) of different polymeric assemblies.

Figure S13. Time-dependent DLS measurement of R73-B73 (co-)assemblies upon 10 mM glutathione treatment.

Figure S14. Time-dependent fluorescence spectra of Nile red within the R37-B73 assemblies (20\% crosslinking).

Figure S15. Time-dependent fluorescence spectra of Nile red within the R55-B73 assemblies (20\% crosslinking).

Figure S16. Time-dependent fluorescence spectra of Nile red within the R73-B73 assemblies (20\% crosslinking).

Figure S17. Time-dependent fluorescence spectra of Nile red within the R91-B73 assemblies (20\% crosslinking).

Figure S18. Nile red release profiles from the R37-B73 assemblies without the presence of glutathione.

Figure S19. Nile red release profiles from the R55-B73 assemblies without the presence of glutathione.

Figure S20. Nile red release profiles from the R73-B73 assemblies without the presence of glutathione.

Figure S21. Nile red release profiles from the R91-B73 assemblies without the presence of glutathione.

Figure S22. Glutathione-triggered Nile red release profiles of polymeric assemblies with different crosslinking degree.

Figure S23. Time-dependent fluorescence spectra of Nile red within the R73-B73 assemblies (5 \% crosslinking).

Figure S24. Time-dependent fluorescence spectra of Nile red within the R73-B73 assemblies (50\% crosslinking).

Figure S25. Cargo encapsulation and glutathione-triggered cargo release profiles of R37-B73 co-assemblies with different degree of crosslinking.

Figure S26. Time-dependent fluorescence spectra of Nile red within the R37-B73 assemblies (5 \% crosslinking).

Figure S27. Time-dependent fluorescence spectra of Nile red within the R37-B73 assemblies (50\% crosslinking).

Figure S28. Glutathione-triggered Nile red release profiles of polymeric assemblies with different disulfide-containing side chains.

Figure S29. Time-dependent fluorescence spectra of Nile red within the R73-B73 assemblies (PEM treated). 
Figure S30. Time-dependent fluorescence spectra of Nile red within the R73-B73 assemblies (TriEG treated).

Figure S31. Cargo encapsulation and glutathione-triggered cargo release profiles of R37-B73 co-assemblies with varied disulfide-containing side chains.

Figure S32. Time-dependent fluorescence spectra of Nile red within the R37-B73 assemblies (PEM treated).

Figure S33. Time-dependent fluorescence spectra of Nile red within the R37-B73 assemblies (TriEG treated).

Figure S34. Biocompatibility evaluation of the co-assemblies.

Figure S35. Photograph of cotton-plugged glass Pasteur pipette.

\section{Supplementary methods}

2.1. Polymer synthesis

2.2. Scanning electron microscopy (SEM)

2.3. FRET evaluation of polymeric co-assemblies between $\mathbf{R} 73$ and $\mathbf{B} 73$

2.4. Molecular weight measurement of polymeric (co-)assemblies using static light scattering (SLS)

2.5. Evaluation of critical aggregation concentration of polymeric (co-)assemblies

2.6. Cell culture

2.7. Detection of cellular ATP level and evaluation of cellular metabolic activity

\section{Spectral data}

4. References 


\section{Supplementary Figures and Table}
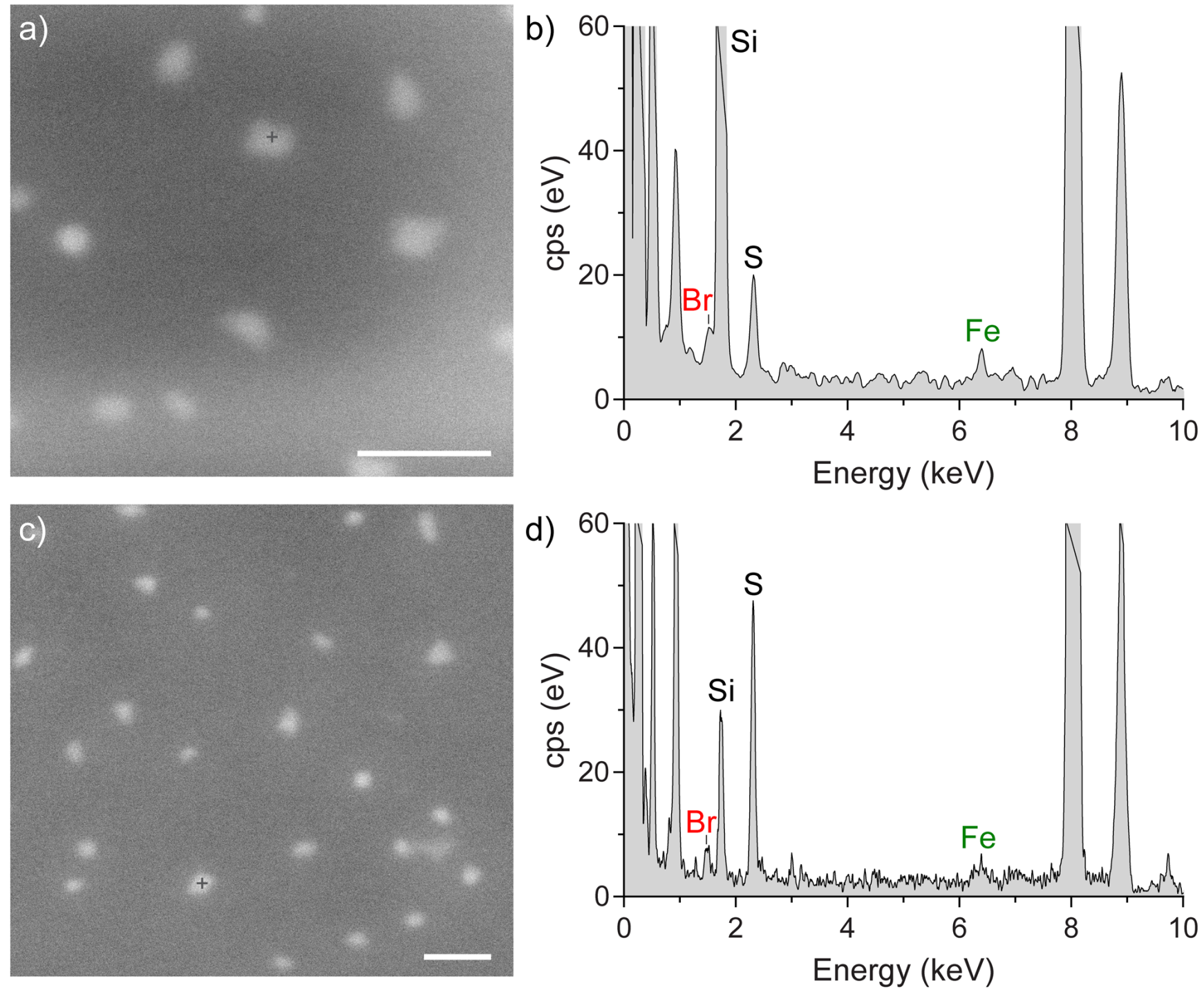

Figure S1. (a,c) High-angle annular dark-field scanning TEM (HAADF-STEM) image and (b,d) the corresponding energy-dispersive X-ray spectroscopic analysis of co-assemblies between $\mathbf{R 7 3 - B r}$ and B73-Fe. Contamination including silicon could be reduced from $(a, b)$ to $(c, d)$ through application of a 15minute beam shower, which did not have negative impact on both $\mathrm{Br}$ and Fe detection. The $\mathbf{R 7 3 - B r - B 7 3 -}$ Fe $(w / w=1: 1)$ co-assemblies were treated with 10 mol\% (vs. PDS units) of DTT to aim at 20 mol\% crosslinking of the PDS units. The scale bars in each figure represents $100 \mathrm{~nm}$. 


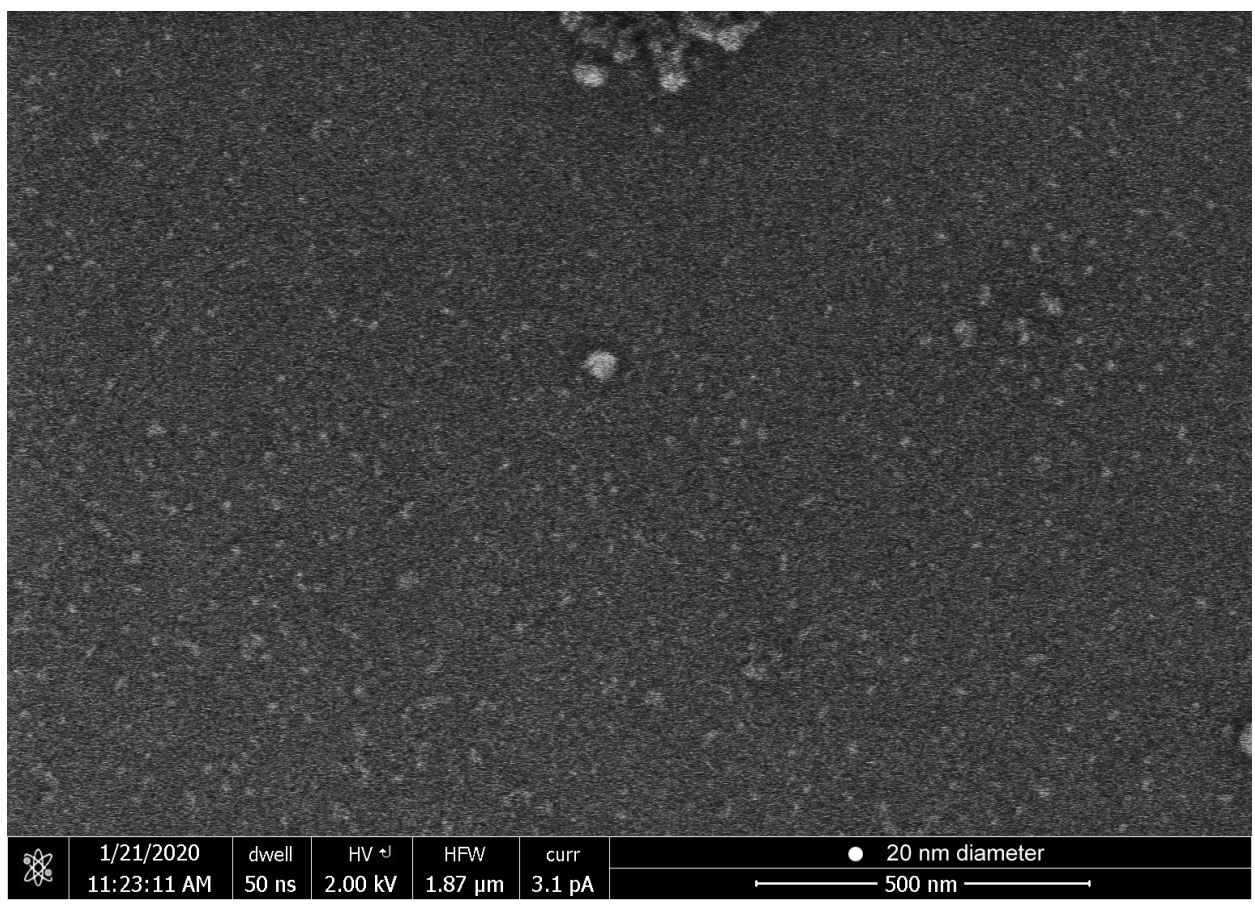

Figure S2. Scanning electron microscopy (SEM) image of co-assemblies between R73 and B73 $(w / w=$ 1:1). 
a)

i) Mix in acetone

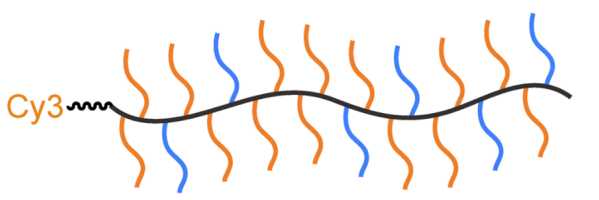

ii) Co-assemble in water

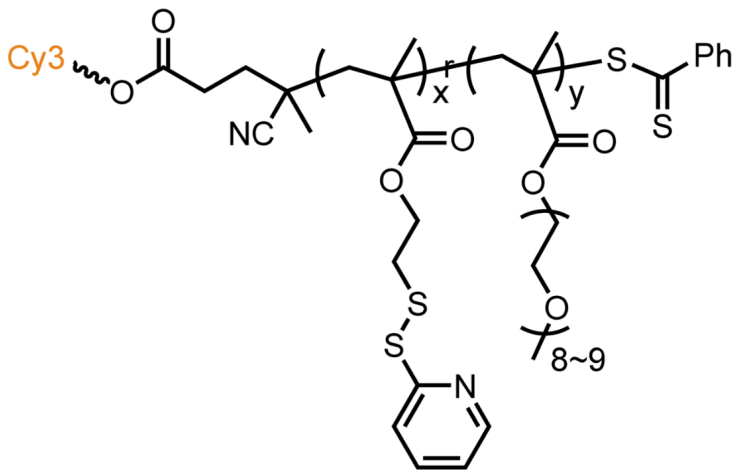

R73-Cy3

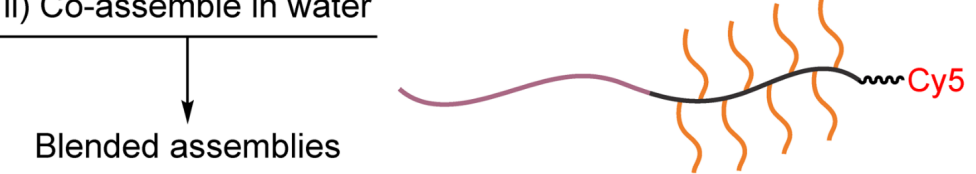

Blended assemblies

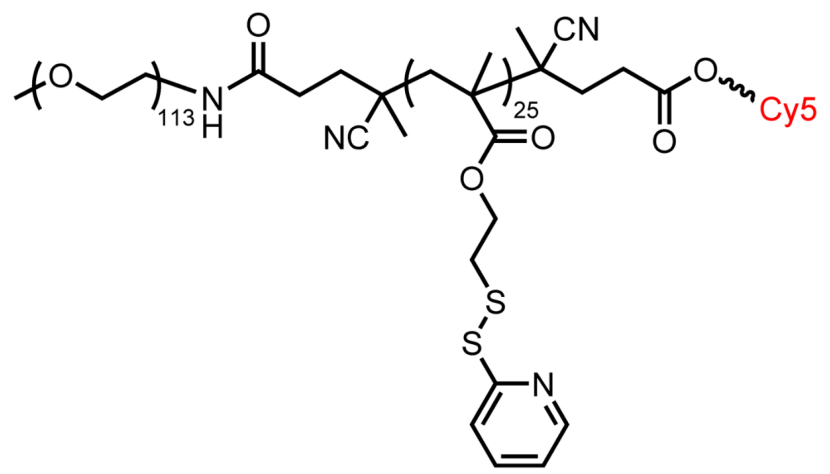

B73-Cy5
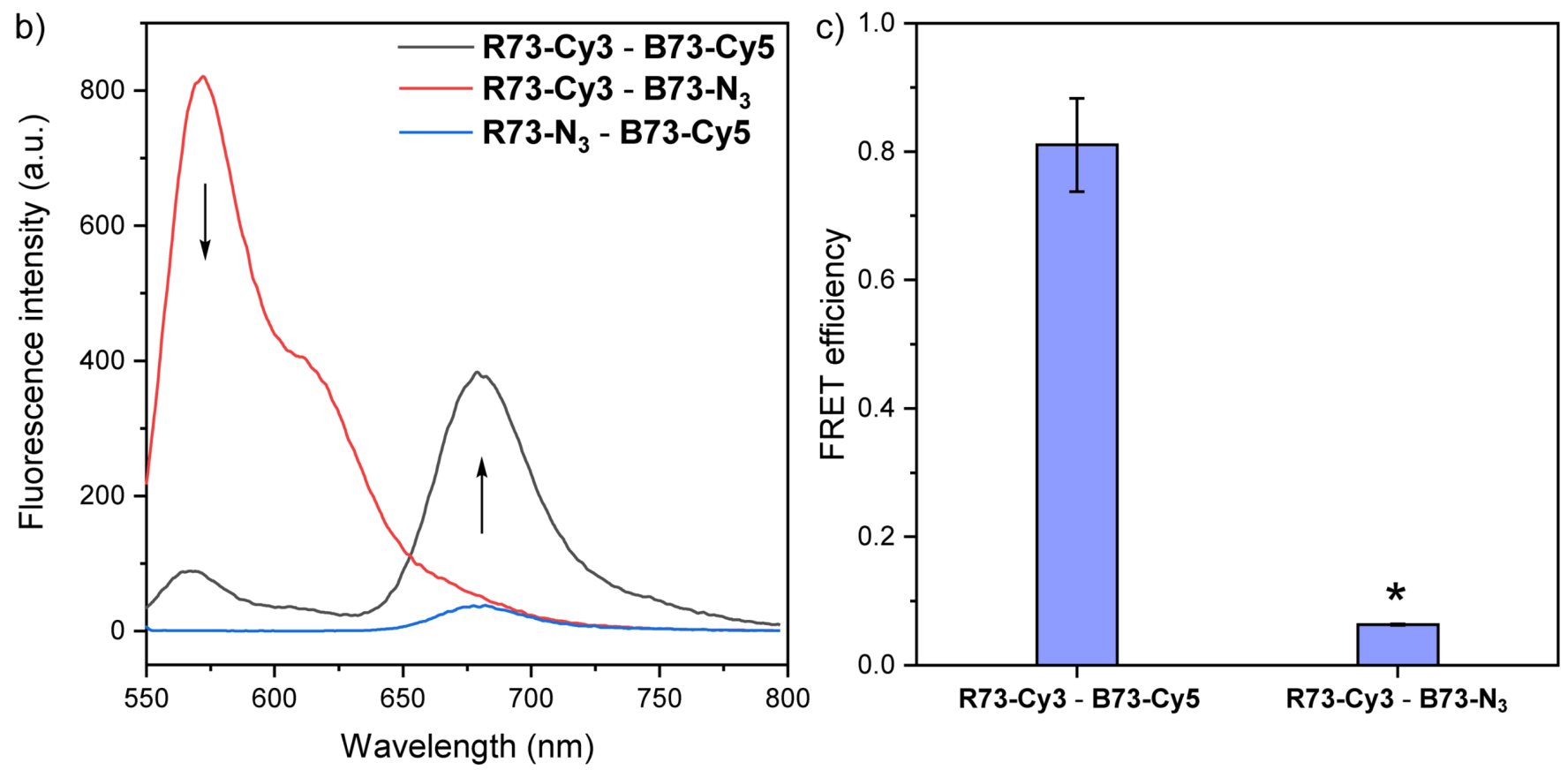

Figure S3. (a) Formulation process of co-assemblies between R73-Cy3 and B73-Cy5. (b) Fluorescence

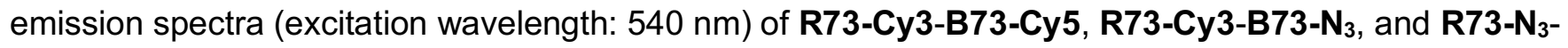
B73-Cy5 co-assemblies $(w / w=1: 1)$. (c) FRET efficiency $\left[/_{c y 5} /\left(I_{c y 3}+I_{c y 5}\right)\right]$ comparison between R73-Cy3B73-Cy5 and R73-Cy3-B73-N 3 co-assemblies (w/w = 1:1). $n=3 .{ }^{*}, P<0.01$. 

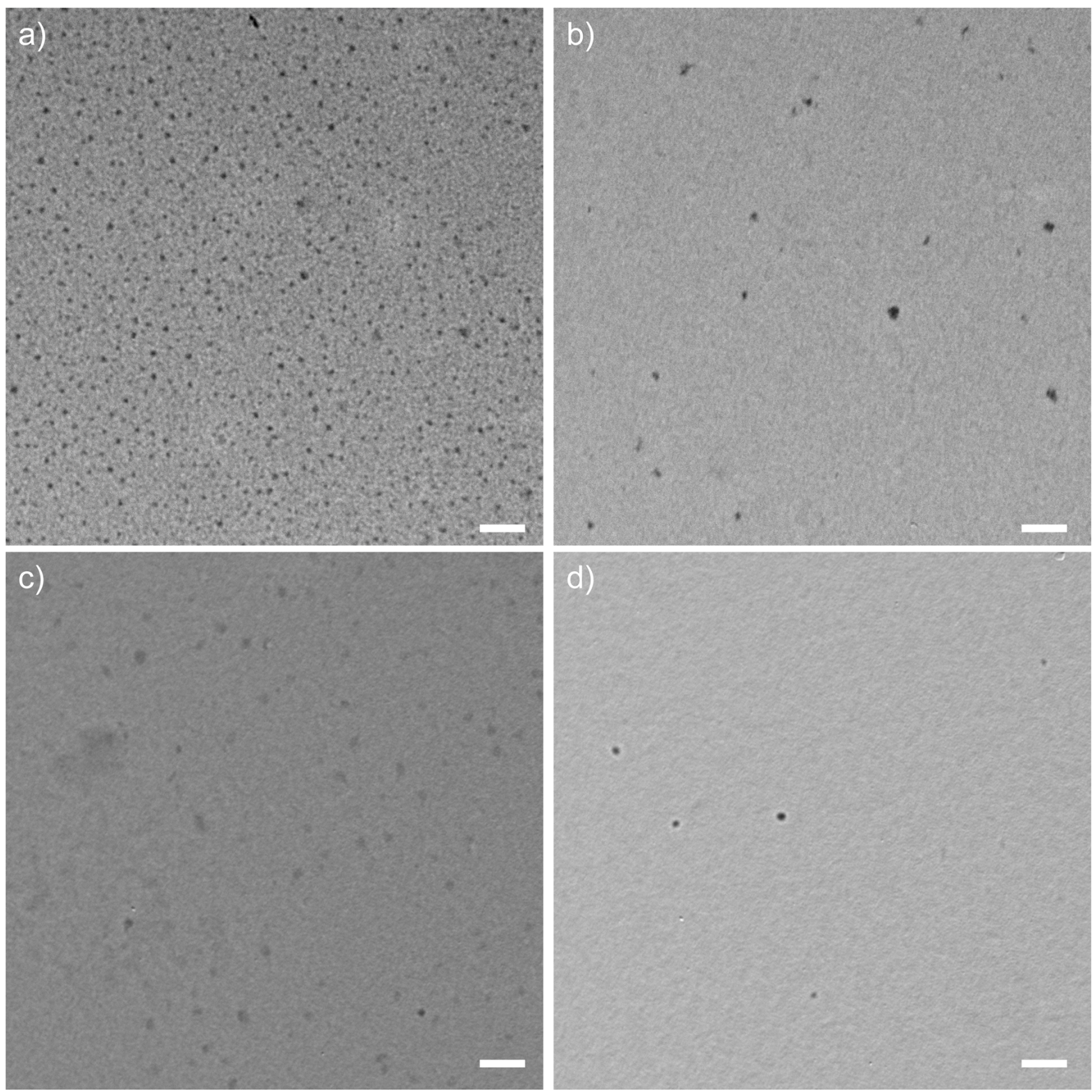

Figure S4. Dry-state TEM images of (co-)assemblies between R73-Br and B73-Fe: (a) w/w = 1:0, (b) $w / w=3: 1$, (c) $w / w=1: 3$, and (d) $w / w=0: 1$. Scale bars represent $100 \mathrm{~nm}$. 

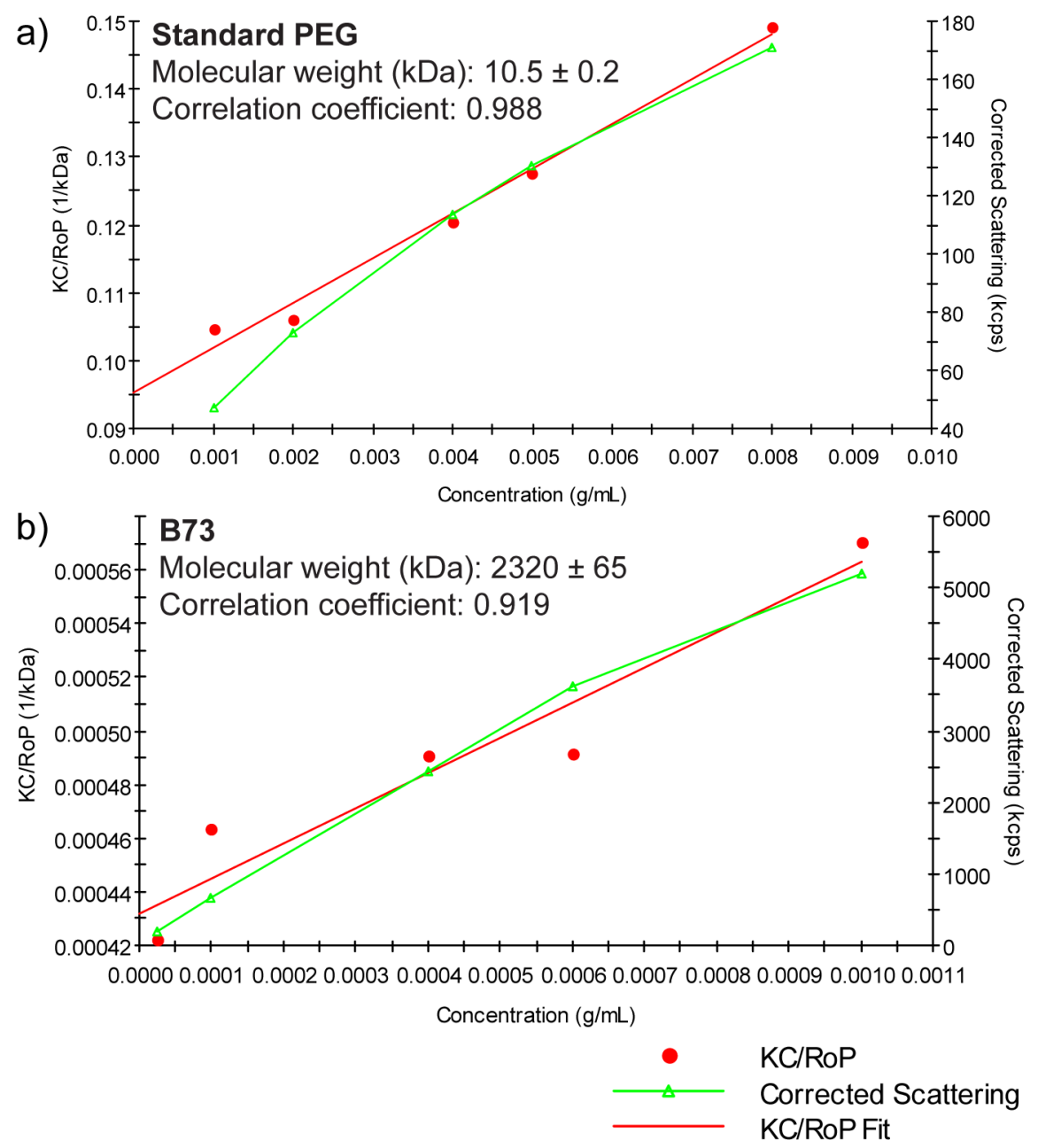

Figure S5. Debye plots from static light scattering for (a) standard PEG $\left(1 \mathrm{mg} \cdot \mathrm{mL}^{-1}\right)$ and (b) B73 selfassemblies (1 $\mathrm{mg} \cdot \mathrm{mL}^{-1}$, treated with DTT to aim at $20 \mathrm{~mol} \%$ crosslinking of the PDS units) in water. The molecular weight analysis from the Debye plot for the standard PEG agrees with the characterization results from the manufacturer (Agilent Technologies, batch No. 0006312704, $M_{\mathrm{p}}=10,370 \mathrm{~g} \cdot \mathrm{mol}^{-1}, \Xi=$ 1.04), confirming the validity of the instrumentation for molecular weight measurement. 

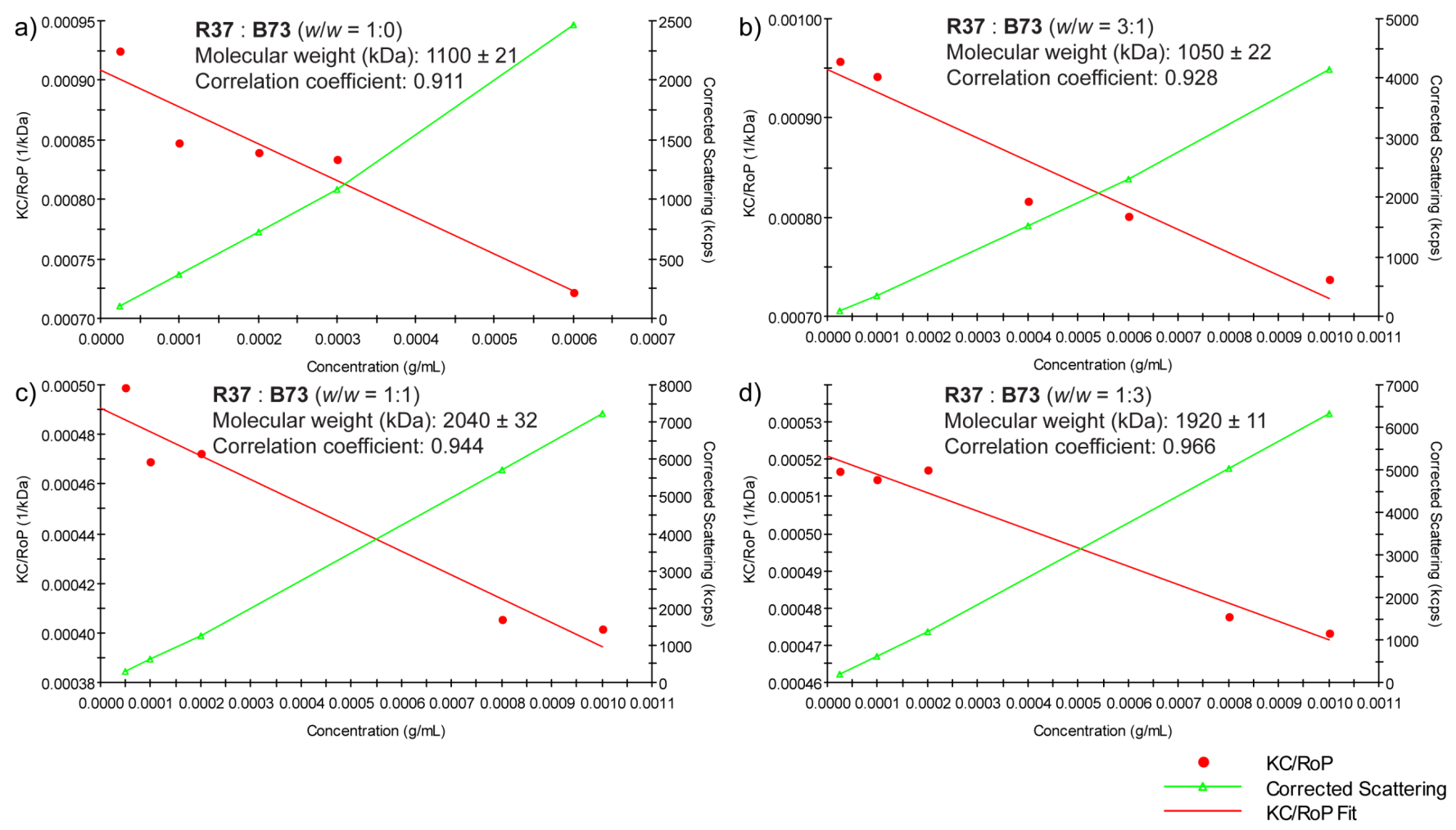

Figure S6. Debye plots from static light scattering for (a) R37, (b) R37 and B73 (w/w = 3:1), (c) R37 and B73 ( $w / w=1: 1)$, and (d) R37 and B73 ( $w / w=1: 3)$ assemblies $\left(1 \mathrm{mg} \cdot \mathrm{mL}^{-1}\right.$ in water). The copolymers in each group were treated with $10 \mathrm{~mol} \%$ (vs. PDS units) of DTT to aim at $20 \mathrm{~mol} \%$ crosslinking of the PDS units. 

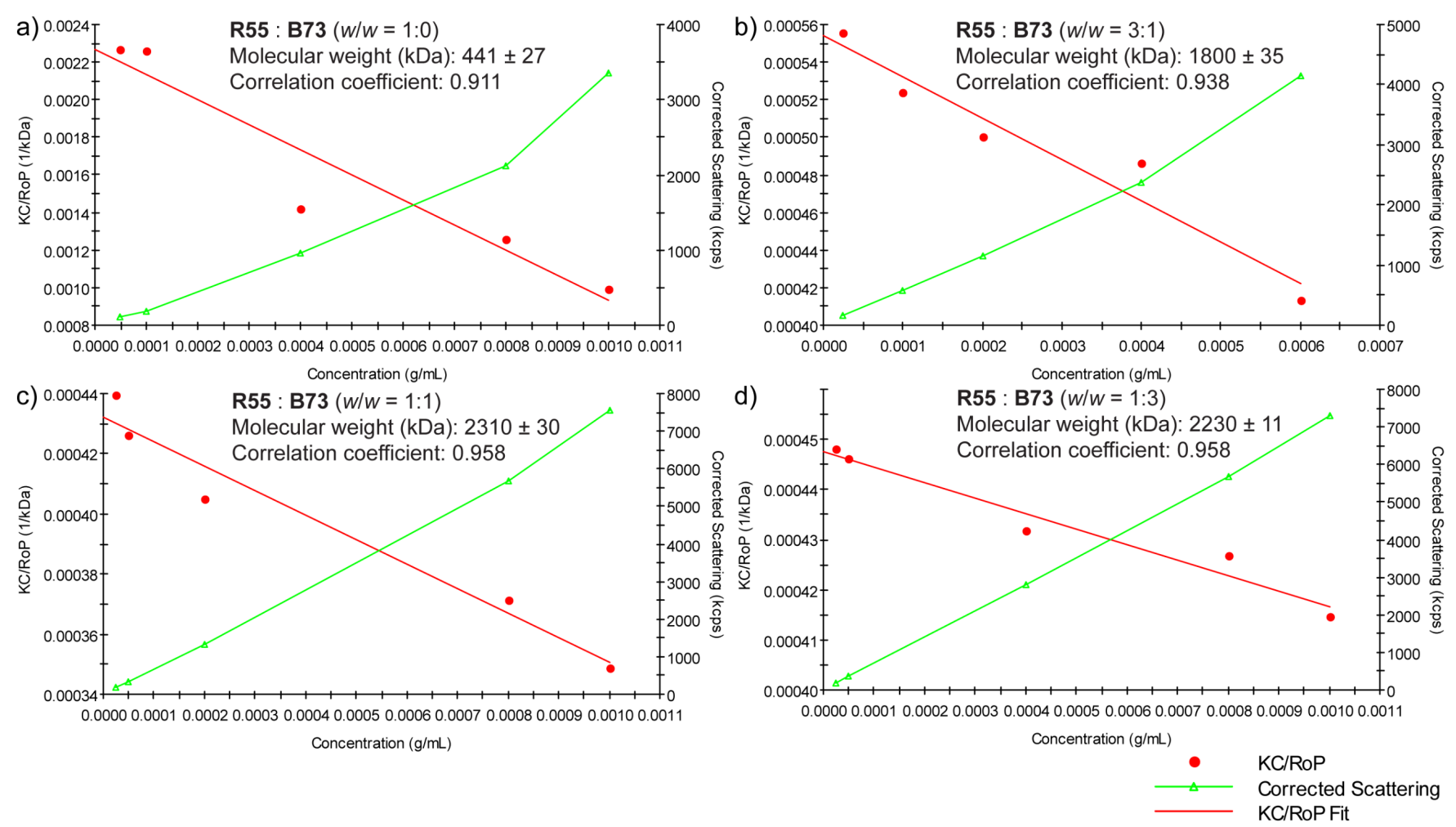

Figure S7. Debye plots from static light scattering for (a) R55, (b) R55 and B73 (w/w = 3:1), (c) R55 and B73 $(w / w=1: 1)$, and (d) R55 and B73 ( $w / w=1: 3)$ assemblies $\left(1 \mathrm{mg} \cdot \mathrm{mL}^{-1}\right.$ in water). The copolymers in each group were treated with $10 \mathrm{~mol} \%$ (vs. PDS units) of DTT to aim at $20 \mathrm{~mol} \%$ crosslinking of the PDS units. 

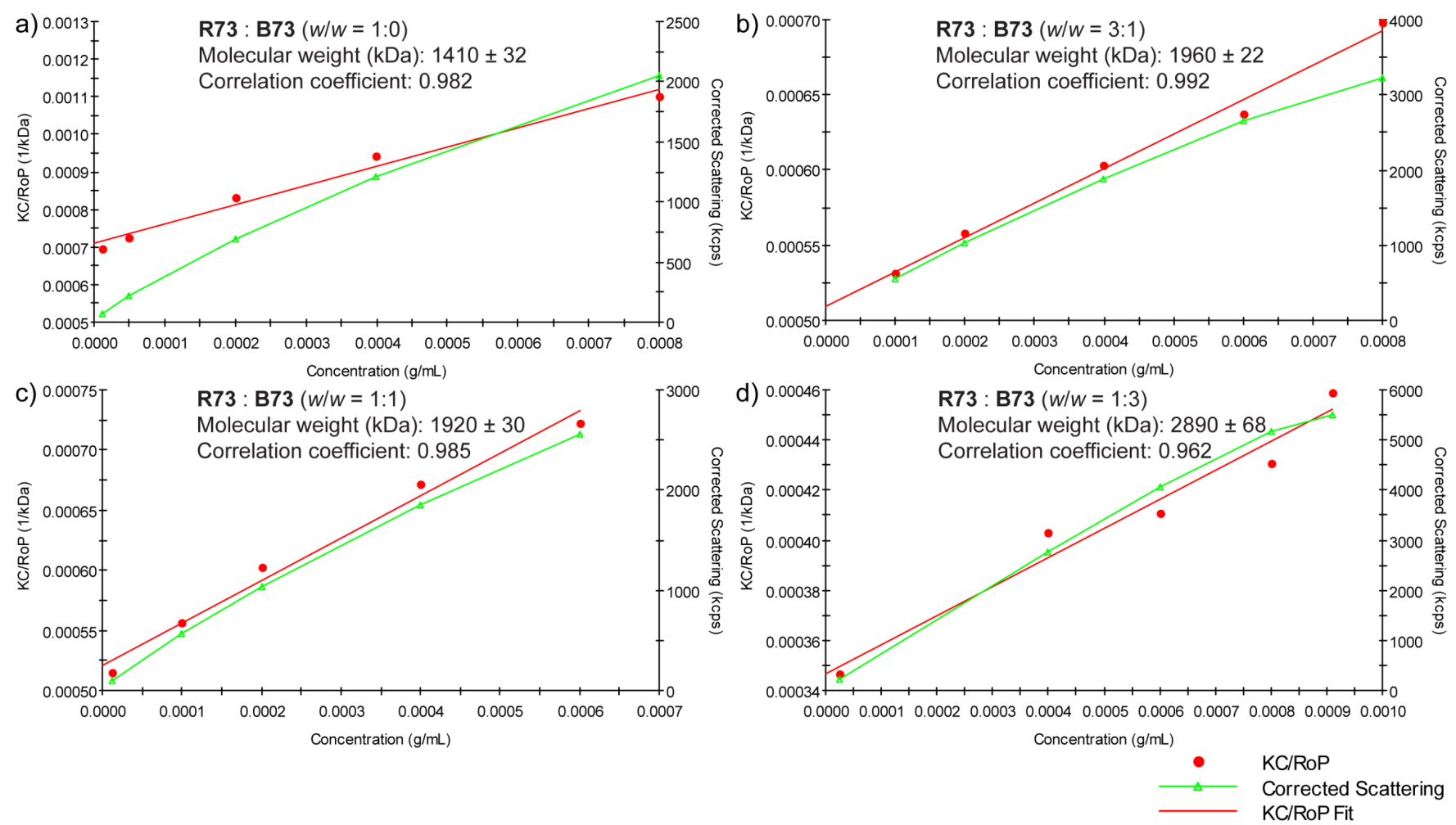

Figure S8. Debye plots from static light scattering for (a) R73, (b) R73 and B73 (w/w = 3:1), (c) R73 and B73 $(w / w=1: 1)$, and (d) R73 and B73 ( $w / w=1: 3)$ assemblies $\left(1 \mathrm{mg} \cdot \mathrm{mL}^{-1}\right.$ in water). The copolymers in each group were treated with $10 \mathrm{~mol} \%$ (vs. PDS units) of DTT to aim at $20 \mathrm{~mol} \%$ crosslinking of the PDS units. 

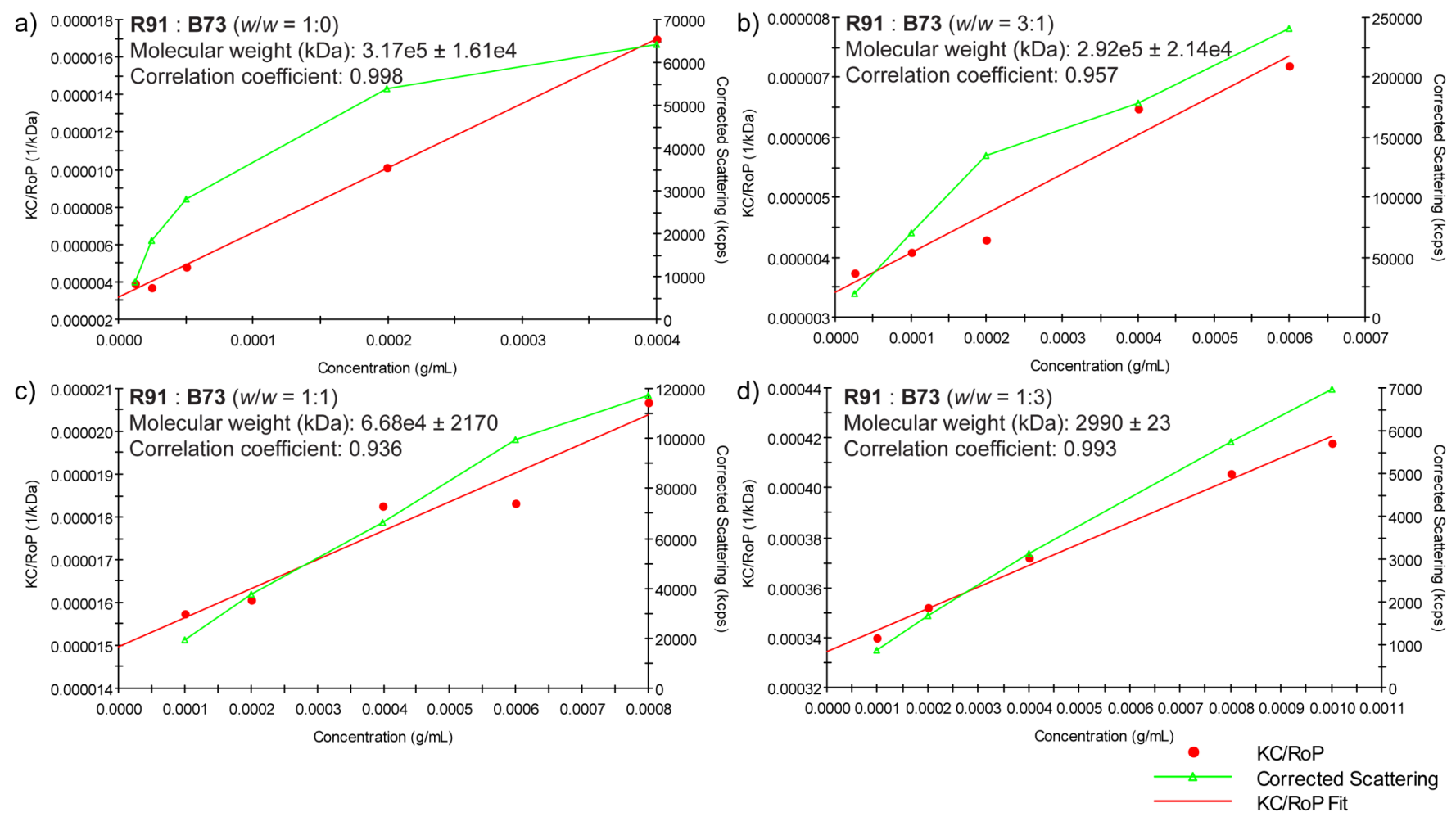

Figure S9. Debye plots from static light scattering for (a) R91, (b) R91 and B73 (w/w = 3:1), (c) R91 and B73 ( $w / w=1: 1)$, and (d) R91 and B73 ( $w / w=1: 3)$ assemblies $\left(1 \mathrm{mg} \cdot \mathrm{mL}^{-1}\right.$ in water). The copolymers in each group were treated with $10 \mathrm{~mol} \%$ (vs. PDS units) of DTT to aim at $20 \mathrm{~mol} \%$ crosslinking of the PDS units. Note that for the static light scattering that is conducted by Malvern Zetasizer Nano, the upper limit for accurate molecular weight measurement is $4,000 \mathrm{kDa}$. Thus, the results for (a) $\mathbf{R 9 1}$, (b) $\mathbf{R} 91$ and $\mathbf{B 7 3}$ ( $w / w=3: 1)$, and (c) R91 and B73 ( $w / w=1: 1)$ will not be considered for the aggregation number comparison in Figure S10. 


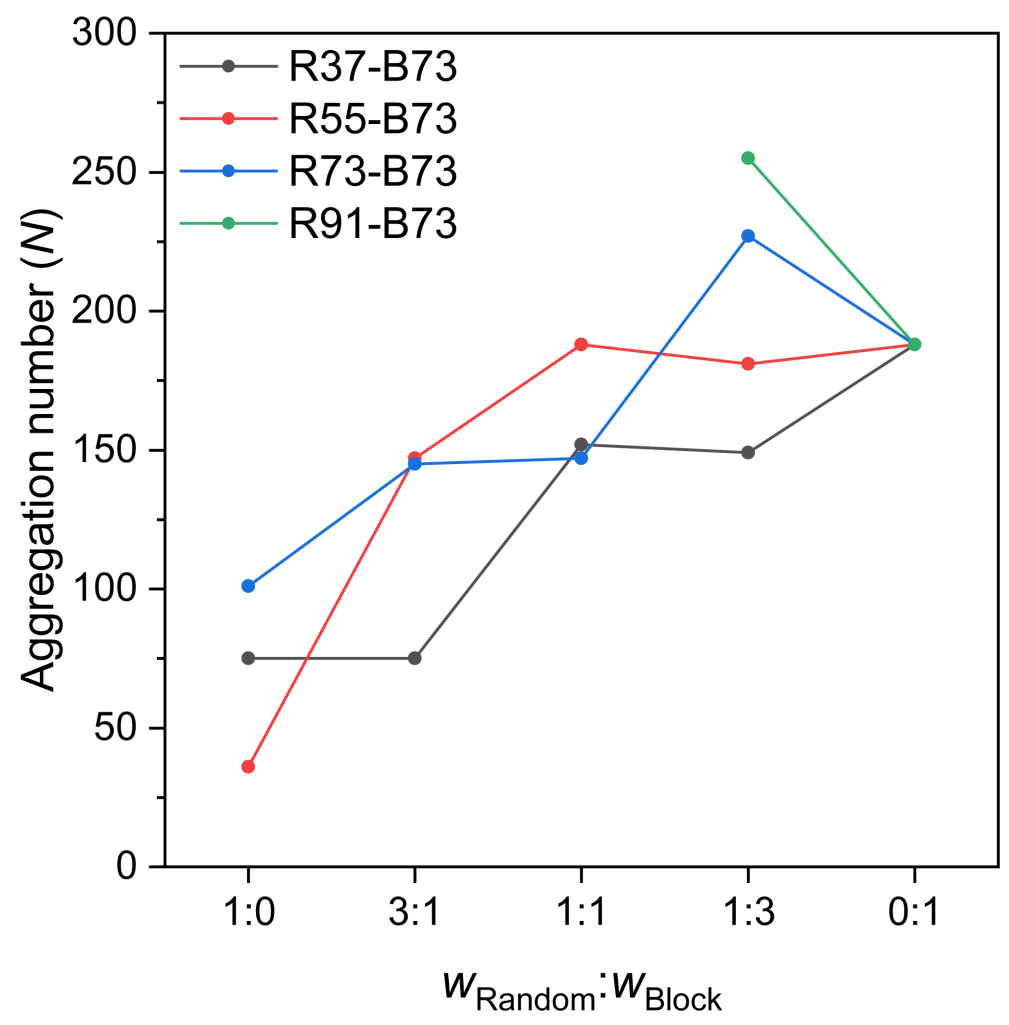

Figure S10. Aggregation number of each (co-)assemblies at different mass ratios. The copolymers (1 $\mathrm{mg} \cdot \mathrm{mL}^{-1}$ in water) in each group were treated with $10 \mathrm{~mol} \%$ (vs. PDS units) of DTT to aim at $20 \mathrm{~mol} \%$ crosslinking of the PDS units. In R37-B73, R55-B73, and R73-B73 series, the aggregation number of each assembly is understandably shifting towards the value of the dominant polymer species within the assemblies. In R91-B73 series, the 1:0, 3:1, and 1:1 (w/w) group are excluded from the comparison as their static light scattering (SLS) results exceed the accuracy limit of the instrumentation. Interestingly, at $W_{\text {Random }}: W_{\text {Block }}=1: 3$, the aggregation number of each co-assemblies increases as the overall HLB of the assembly increases, suggesting that the HLB of polymeric assemblies may also affect the aggregation number of the assembly. As the hydrophobic portion of polymeric assemblies increases, the need for stabilizing these hydrophobic units of amphiphiles increases, indicating larger aggregation number. ${ }^{1}$ Aggregation number $=($ Calibrated molecular weight of crosslinked assemblies based on SLS results / Weight average molecular weight of copolymers based on THF GPC results). 
Table S1. Loading capacity and encapsulation efficiency of Nile red in R73 and B73 with optimized formulation protocols. Briefly, a calculated amount of polymer, Nile red, and dithiothreitol were dissolved in $200 \mu \mathrm{L}$ acetone to aim at $20 \%$ crosslinking within the polymer. After stirring for 10 minutes, $1 \mathrm{~mL}$ deionized $\mathrm{H}_{2} \mathrm{O}$ was added dropwise into the acetone solution. The mixture was stirred overnight with open cap to allow the evaporation of acetone. Next, the aqueous solution was filtered through a cottonfilled glass pipette to remove the insoluble dyes. The residual solution was lyophilized and re-dissolved in DMSO (containing 1 vol\% $\mathrm{H}_{2} \mathrm{O}$ ) for UV spectroscopic analysis. All data are presented as the average \pm standard deviation of three replicates.

\begin{tabular}{ccccc}
\hline $\begin{array}{c}\text { Polymer concentration } \\
\left(\mathbf{m g} \cdot \mathbf{m L}^{-1}\right)\end{array}$ & $\begin{array}{c}\text { Feed loading } \\
(\mathbf{w t} \%)\end{array}$ & $\begin{array}{c}\text { Loading } \\
\text { capacity (\%) }\end{array}$ & $\begin{array}{c}\text { Encapsulation } \\
\text { efficiency (\%) }\end{array}$ \\
\hline R73 & 1.0 & 10 & $6.0 \pm 0.4$ & $64.0 \pm 4.4$ \\
\hline & 0.5 & 10 & $2.6 \pm 0.4$ & $27.1 \pm 4.1$ \\
B73 & 1.0 & 10 & $4.9 \pm 0.3$ & $51.4 \pm 3.6$ \\
& 2.0 & 10 & $3.8 \pm 1.2$ & $39.5 \pm 13.5$ \\
\hline
\end{tabular}



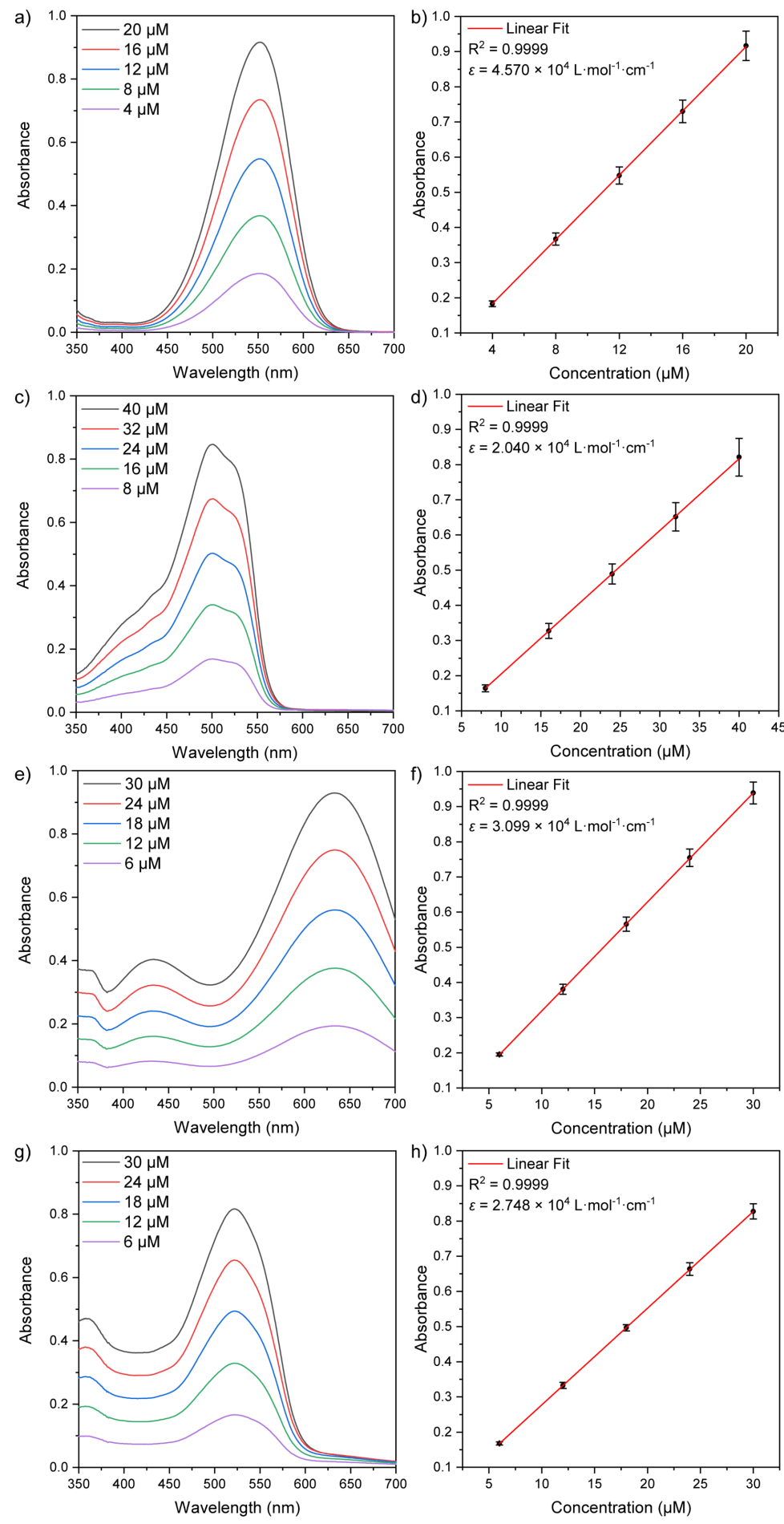

Figure S11. Representative absorption spectra of (a) Nile red, (c) Sudan red G, (e) Sudan black B, and (g) Oil red $\mathrm{O}$ at different concentrations. Standard calibration curve for (b) Nile red $\left(\lambda_{\max }=552 \mathrm{~nm}\right)$, (d) Sudan red G $\left(\lambda_{\max }=501 \mathrm{~nm}\right)$, (f) Sudan black $B\left(\lambda_{\max }=633 \mathrm{~nm}\right)$, and (h) Oil red O $\left(\lambda_{\max }=522 \mathrm{~nm}\right)$. Error bars represent the standard deviation of three replicates. 
a)

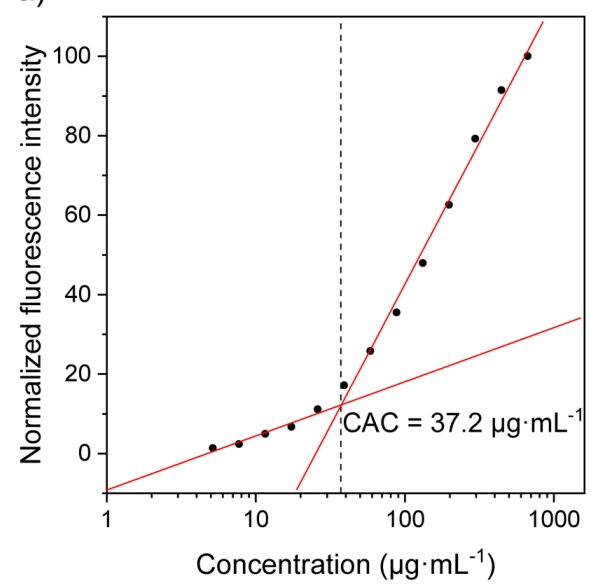

d)

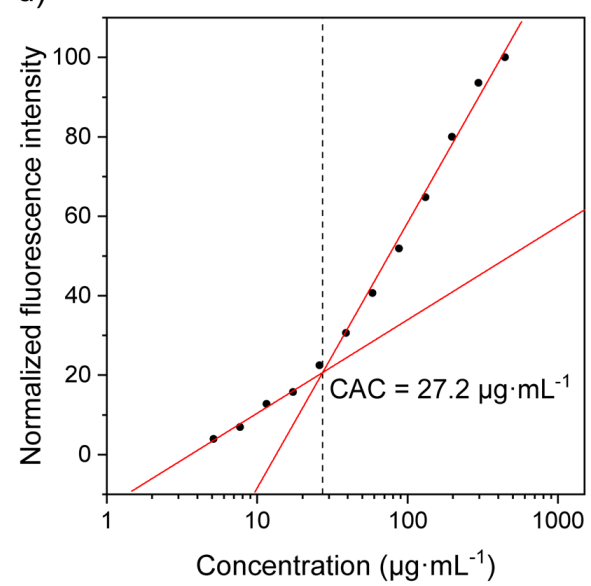

g)

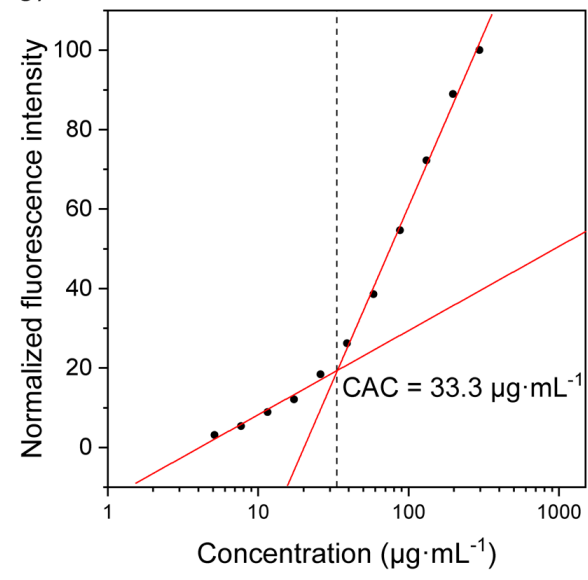

b)

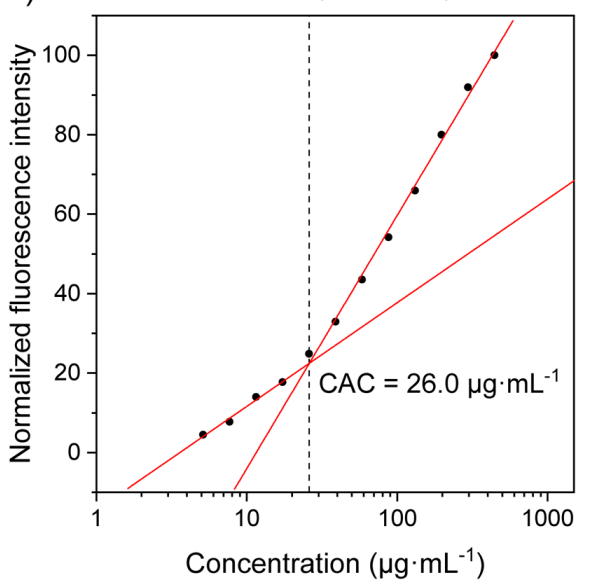

e)

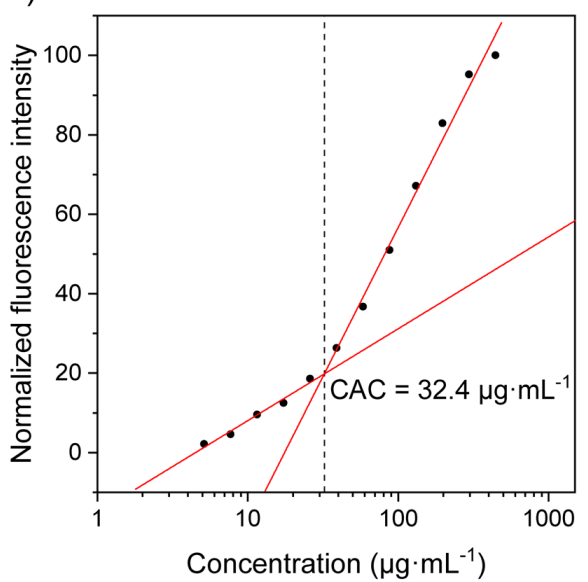

h)

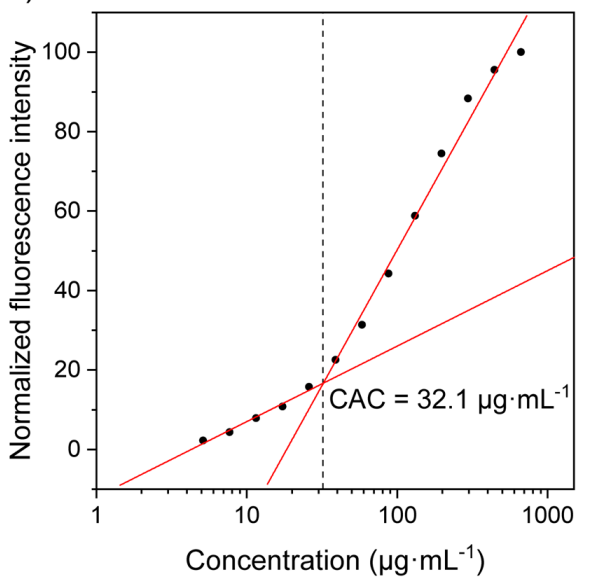

c) $\quad$ R55

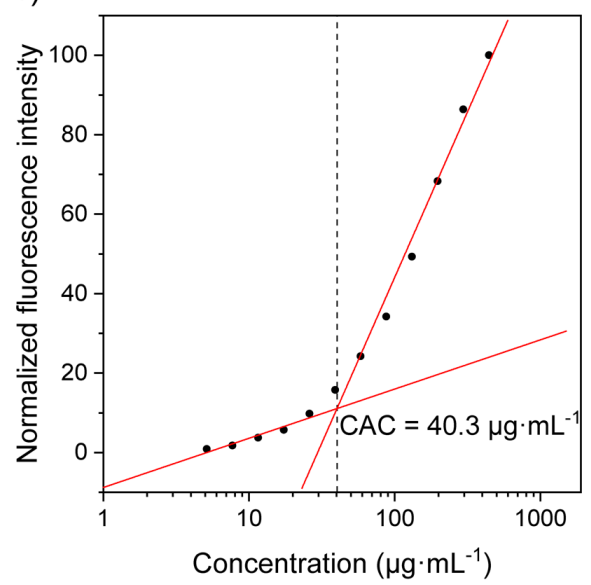

f)

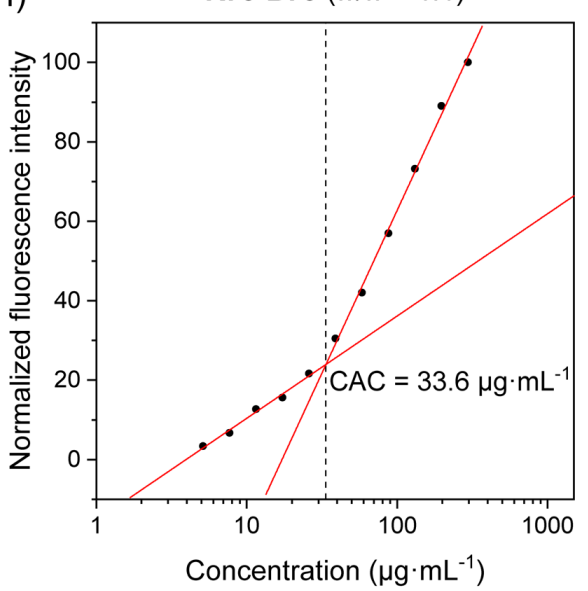

i)

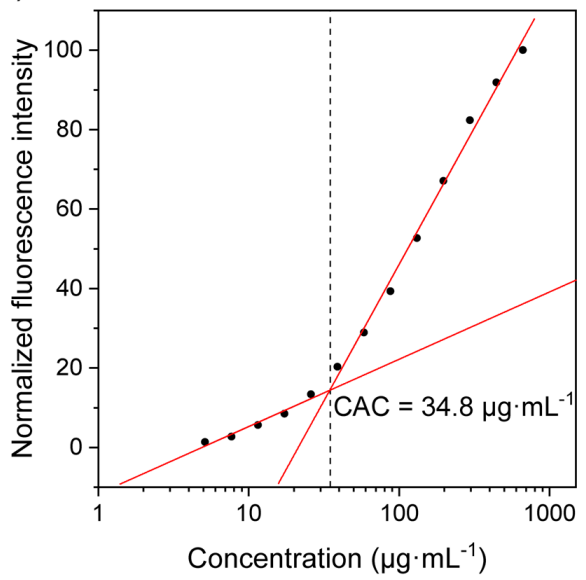

Figure S12. Critical aggregation concentration (CAC) of different polymeric assemblies. The copolymers in each group were treated with $10 \mathrm{~mol} \%$ (vs. PDS units) of DTT to aim at $20 \mathrm{~mol} \%$ crosslinking of the PDS units. The fluorescence emission intensity $(628 \mathrm{~nm})$ of encapsulated Nile red at the maximal tested concentration was normalized as $100 \%$. 

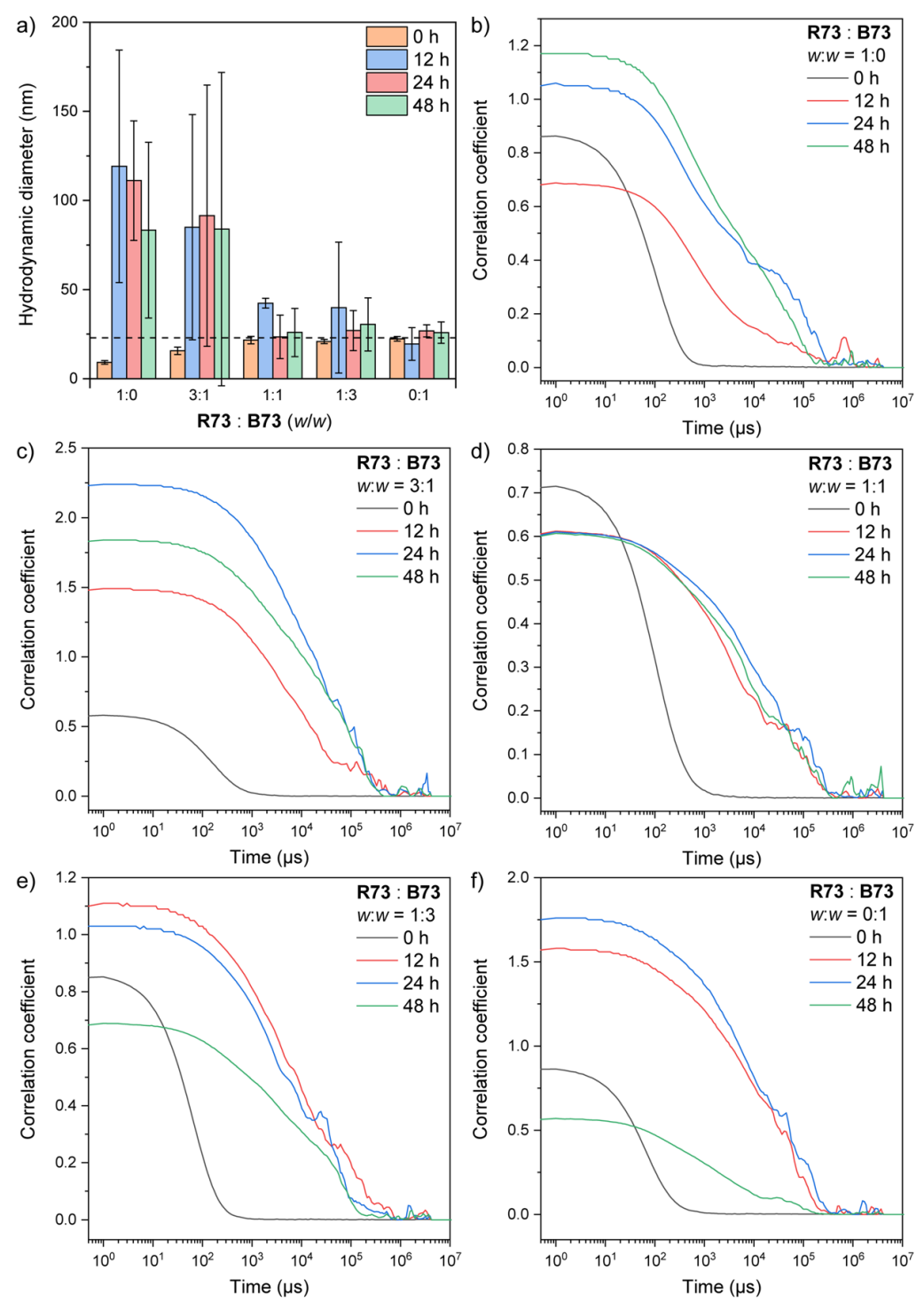

Figure S13. (a) Hydrodynamic diameter evolution of each (co-)assemblies after $10 \mathrm{mM}$ glutathione (GSH) treatment. Dashed line is used as a reference line for each figure, representing the value of 23 $\mathrm{nm}$. Error bars represent the standard deviation of three replicates. (b f) Dynamic light scattering correlograms of (b) R73, (c) R73 and B73 ( $w / w=3: 1$ ), (d) R73 and B73 ( $w / w=1: 1$ ), (e) R73 and B73 $(w / w=1: 3)$, and (f) B73 assemblies. The exponential decay in each graph $0 \mathrm{~h}$ represents the monodispersity within a large number of particles. After treating each (co-)assemblies with $10 \mathrm{mM} \mathrm{GSH}$ at different time points, each sample did not maintain the original exponential decay and lost the monodispersity. Overall, the dataset indicates the change in HLB and dispersity of polymeric assemblies after GSH treatment. 

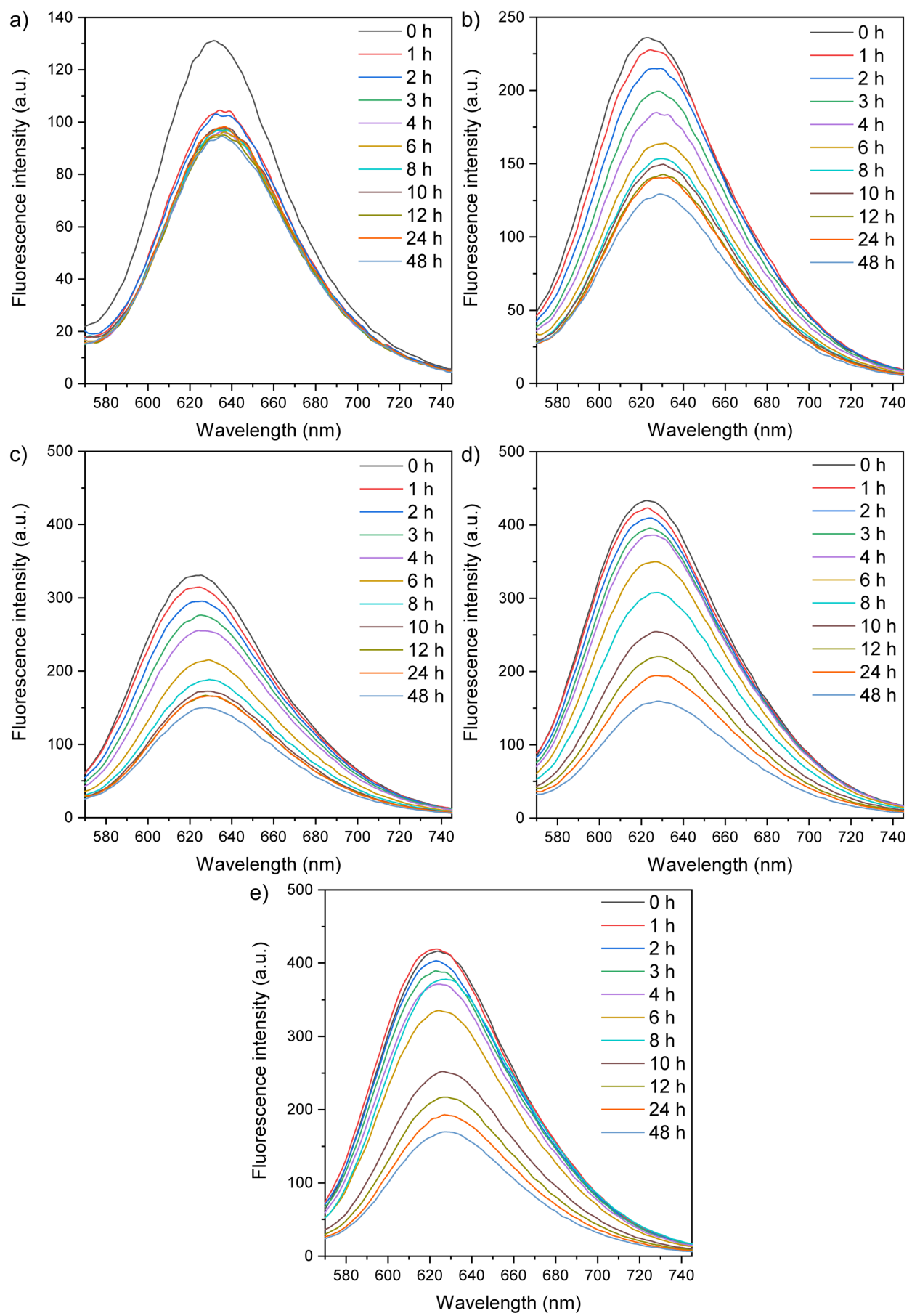

Figure S14. Representative time-dependent fluorescence emission spectra of Nile red within the assemblies of (a) R37, (b) R37 and B73 (w/w = 3:1), (c) R37 and B73 (w/w = 1:1), (d) R37 and B73 (w/w $=1: 3$ ), and (e) B73. The copolymers in each group were treated with 10 mol\% (vs. PDS units) of DTT to aim at $20 \mathrm{~mol} \%$ crosslinking of the PDS units. Glutathione was maintained at a final concentration of 10 $\mathrm{mM}$ to trigger the Nile red release. 

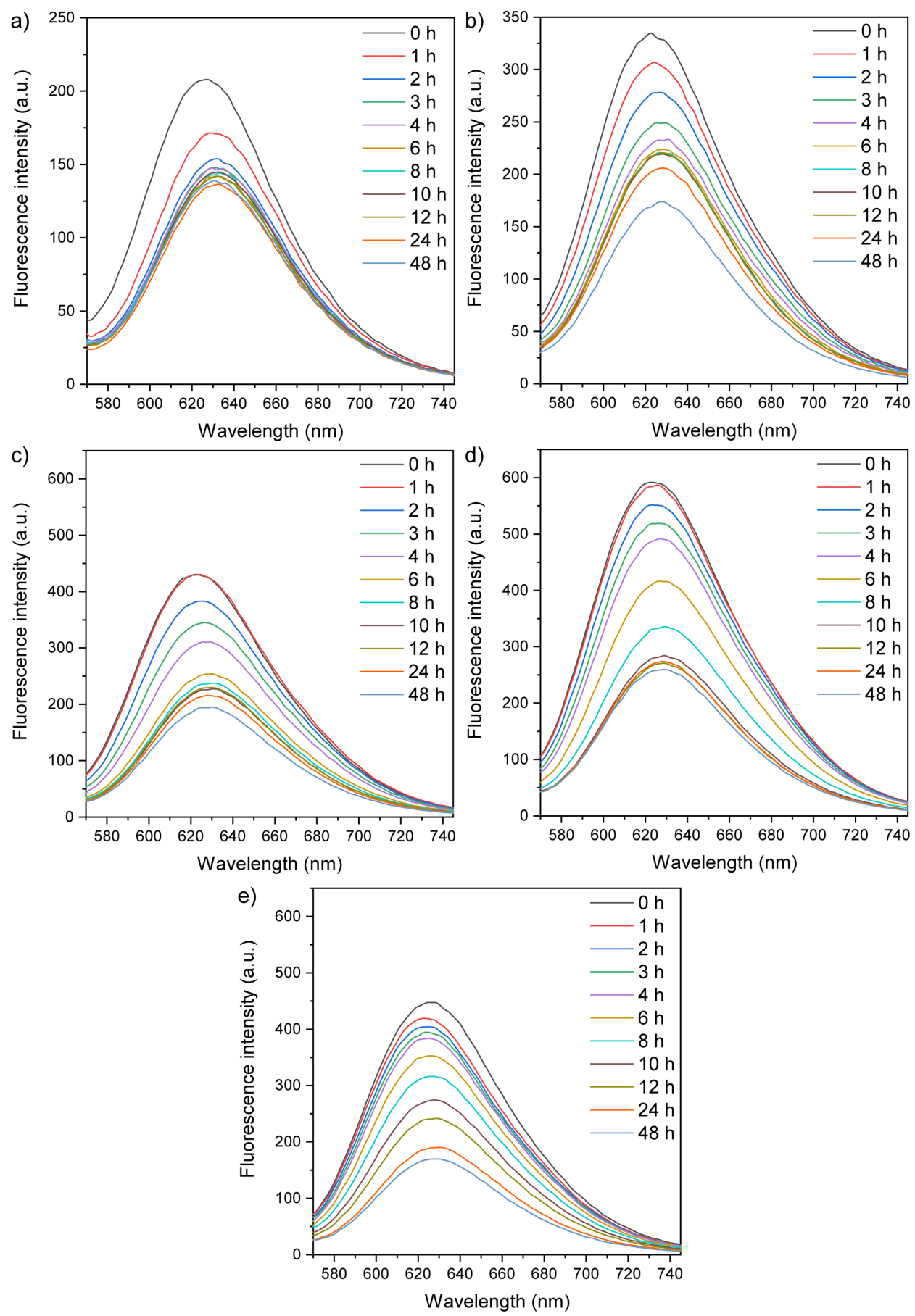

Figure S15. Representative time-dependent fluorescence emission spectra of Nile red within the assemblies of (a) R55, (b) R55 and B73 ( $w / w=3: 1$ ), (c) R55 and B73 (w/w = 1:1), (d) R55 and B73 (w/w $=1: 3$ ), and (e) B73. The copolymers in each group were treated with 10 mol\% (vs. PDS units) of DTT to aim at $20 \mathrm{~mol} \%$ crosslinking of the PDS units. Glutathione was maintained at a final concentration of 10 $\mathrm{mM}$ to trigger the Nile red release. 

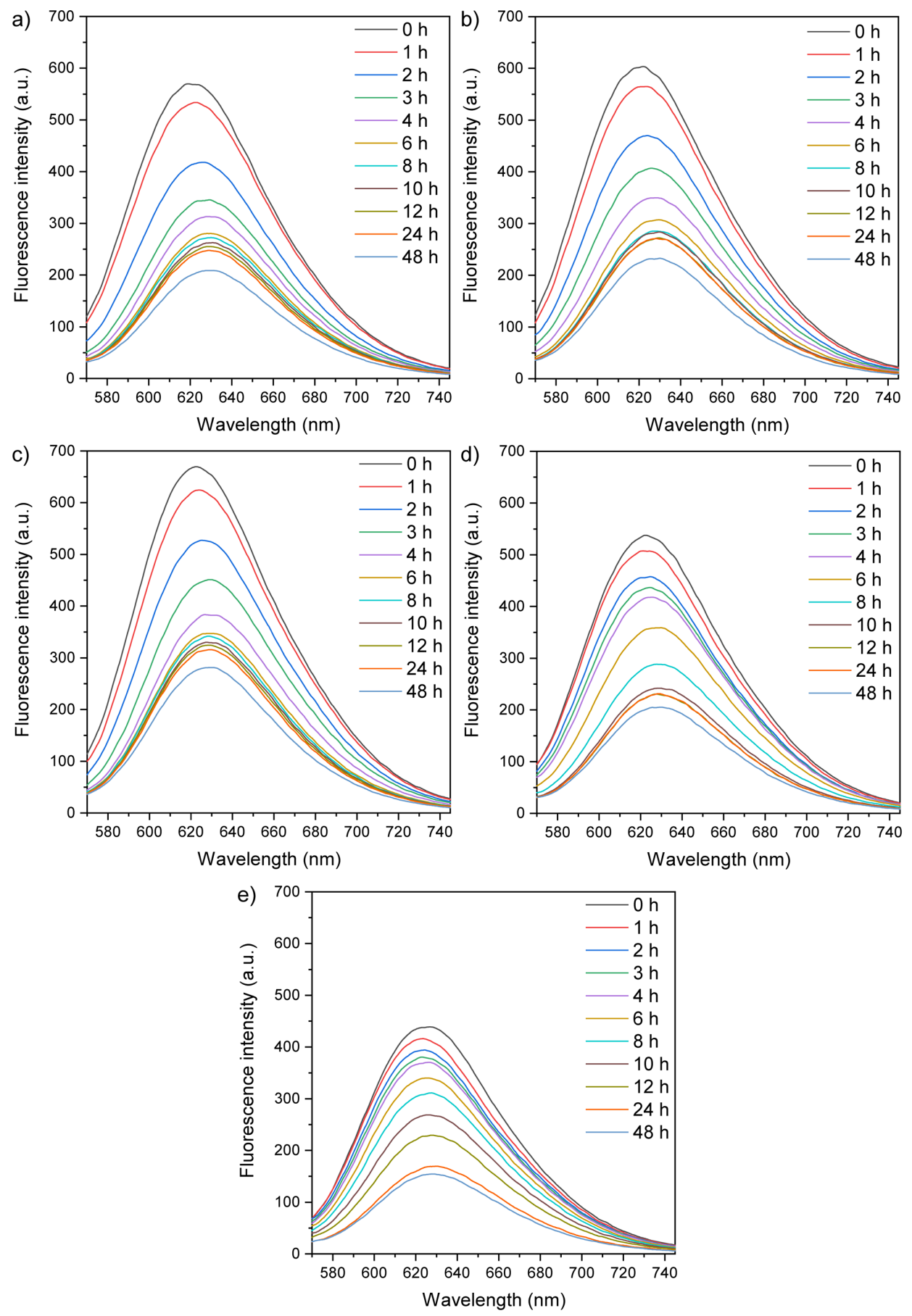

Figure S16. Representative time-dependent fluorescence emission spectra of Nile red within the assemblies of (a) R73, (b) R73 and B73 ( $w / w=3: 1$ ), (c) R73 and B73 (w/w = 1:1), (d) R73 and B73 (w/w $=1: 3$ ), and (e) B73. The copolymers in each group were treated with 10 mol\% (vs. PDS units) of DTT to aim at $20 \mathrm{~mol} \%$ crosslinking of the PDS units. Glutathione was maintained at a final concentration of 10 $\mathrm{mM}$ to trigger the Nile red release. 

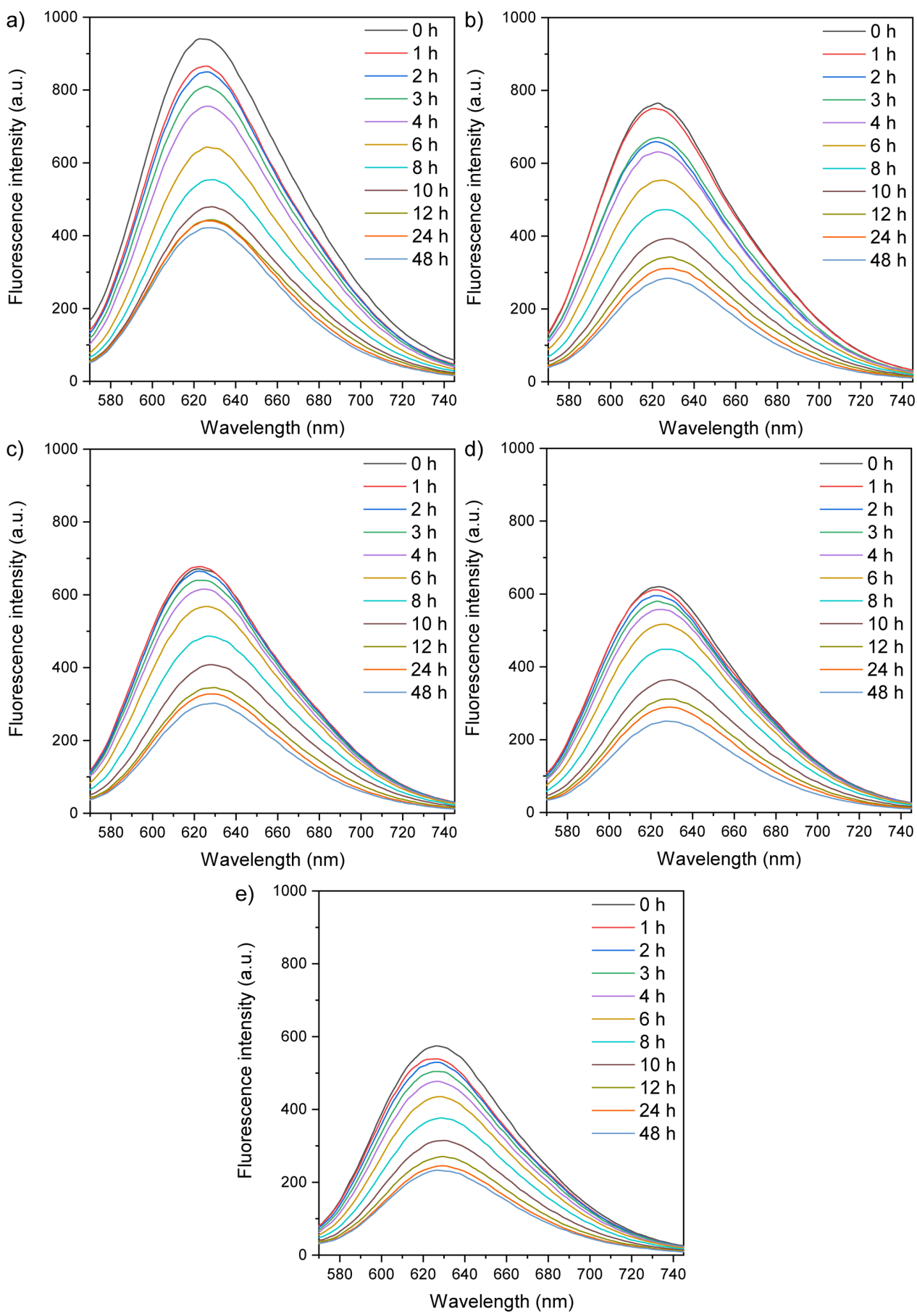

Figure S17. Representative time-dependent fluorescence emission spectra of Nile red within the assemblies of (a) R91, (b) R91 and B73 (w/w = 3:1), (c) R91 and B73 (w/w = 1:1), (d) R91 and B73 (w/w $=1: 3$ ), and (e) B73. The copolymers in each group were treated with 10 mol\% (vs. PDS units) of DTT to aim at $20 \mathrm{~mol} \%$ crosslinking of the PDS units. Glutathione was maintained at a final concentration of 10 $\mathrm{mM}$ to trigger the Nile red release. 

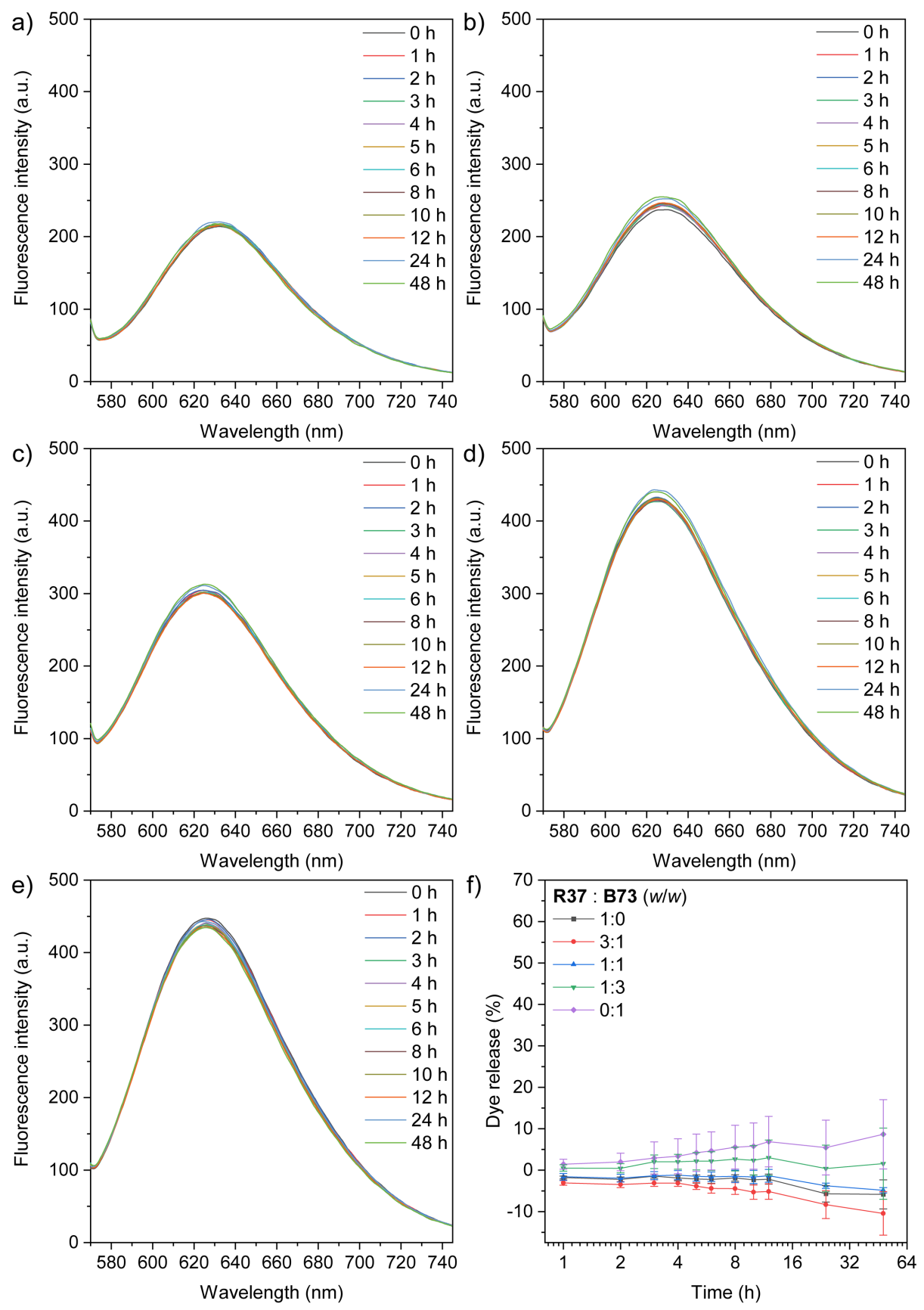

Figure S18. Representative time-dependent fluorescence emission spectra of Nile red within the assemblies of (a) R37, (b) R37 and B73 ( $w / w=3: 1$ ), (c) R37 and B73 ( $w / w=1: 1$ ), (d) R37 and B73 ( $w / w$ $=1: 3$ ), and (e) B73 without the presence of glutathione. The copolymers in each group were treated with 10 mol\% (vs. PDS units) of DTT to aim at 20 mol\% crosslinking of the PDS units. (f) Cargo (Nile red) release profiles of the self-assemblies formed by individual or blended copolymers without the addition of glutathione. 

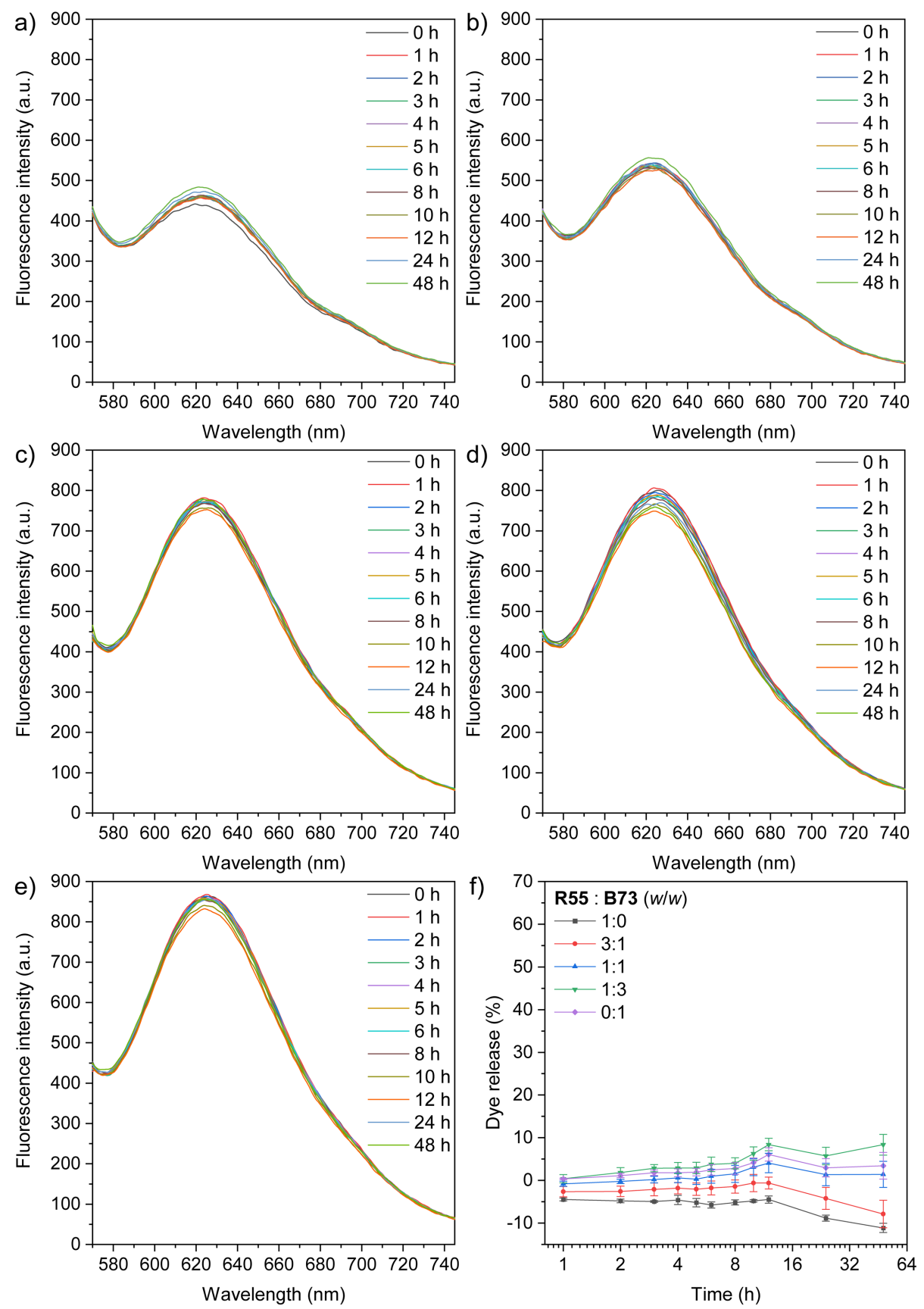

Figure S19. Representative time-dependent fluorescence emission spectra of Nile red within the assemblies of (a) R55, (b) R55 and B73 ( $w / w=3: 1$ ), (c) R55 and B73 ( $w / w=1: 1$ ), (d) R55 and B73 (w/w $=1: 3$ ), and (e) B73 without the presence of glutathione. The copolymers in each group were treated with 10 mol\% (vs. PDS units) of DTT to aim at 20 mol\% crosslinking of the PDS units. (f) Cargo (Nile red) release profiles of the self-assemblies formed by individual or blended copolymers without the addition of glutathione. 

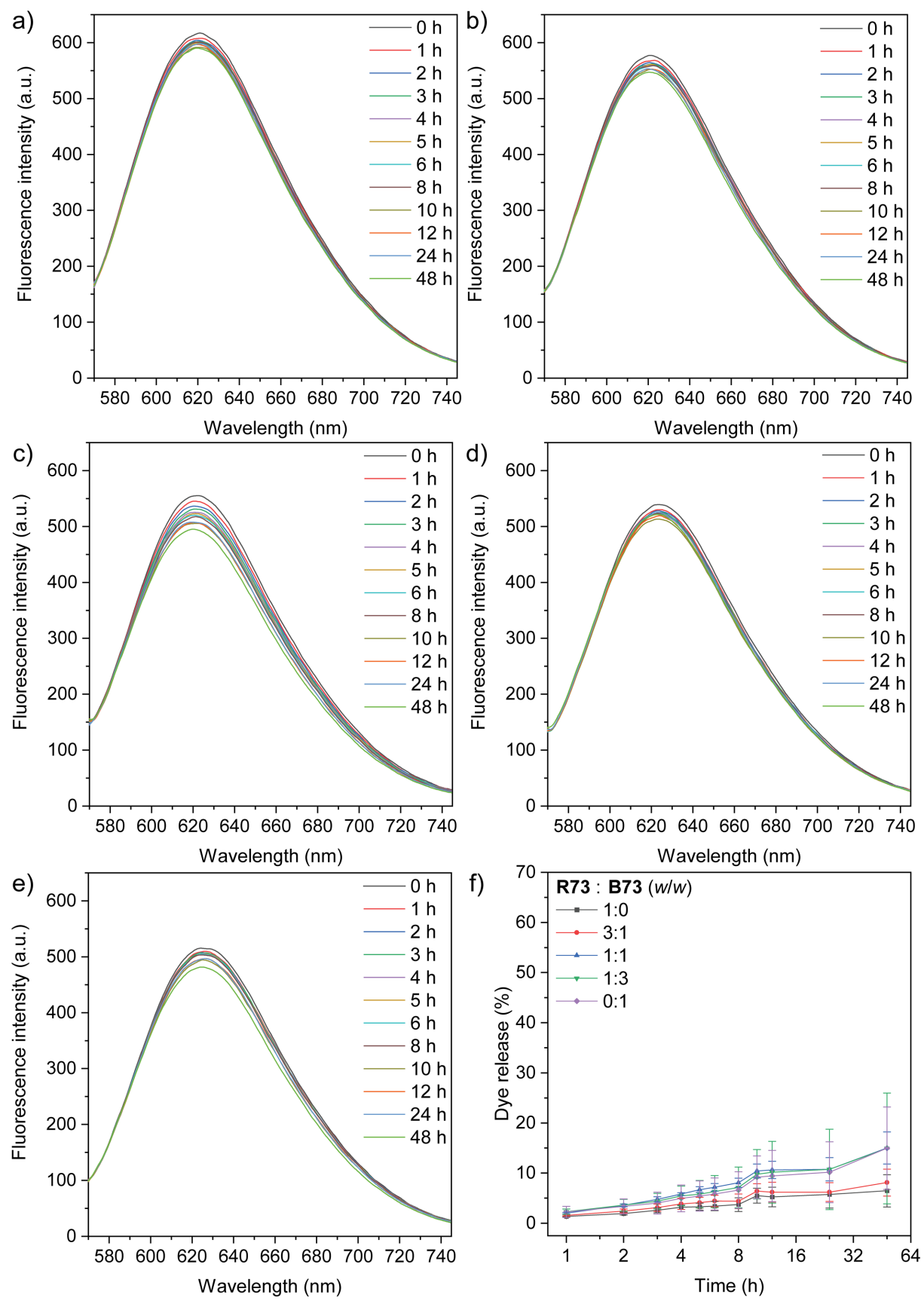

Figure S20. Representative time-dependent fluorescence emission spectra of Nile red within the assemblies of (a) R73, (b) R73 and B73 ( $w / w=3: 1$ ), (c) R73 and B73 ( $w / w=1: 1$ ), (d) R73 and B73 (w/w $=1: 3$ ), and (e) B73 without the presence of glutathione. The copolymers in each group were treated with $10 \mathrm{~mol} \%$ (vs. PDS units) of DTT to aim at $20 \mathrm{~mol} \%$ crosslinking of the PDS units. (f) Cargo (Nile red) release profiles of the self-assemblies formed by individual or blended copolymers without the addition of glutathione. 

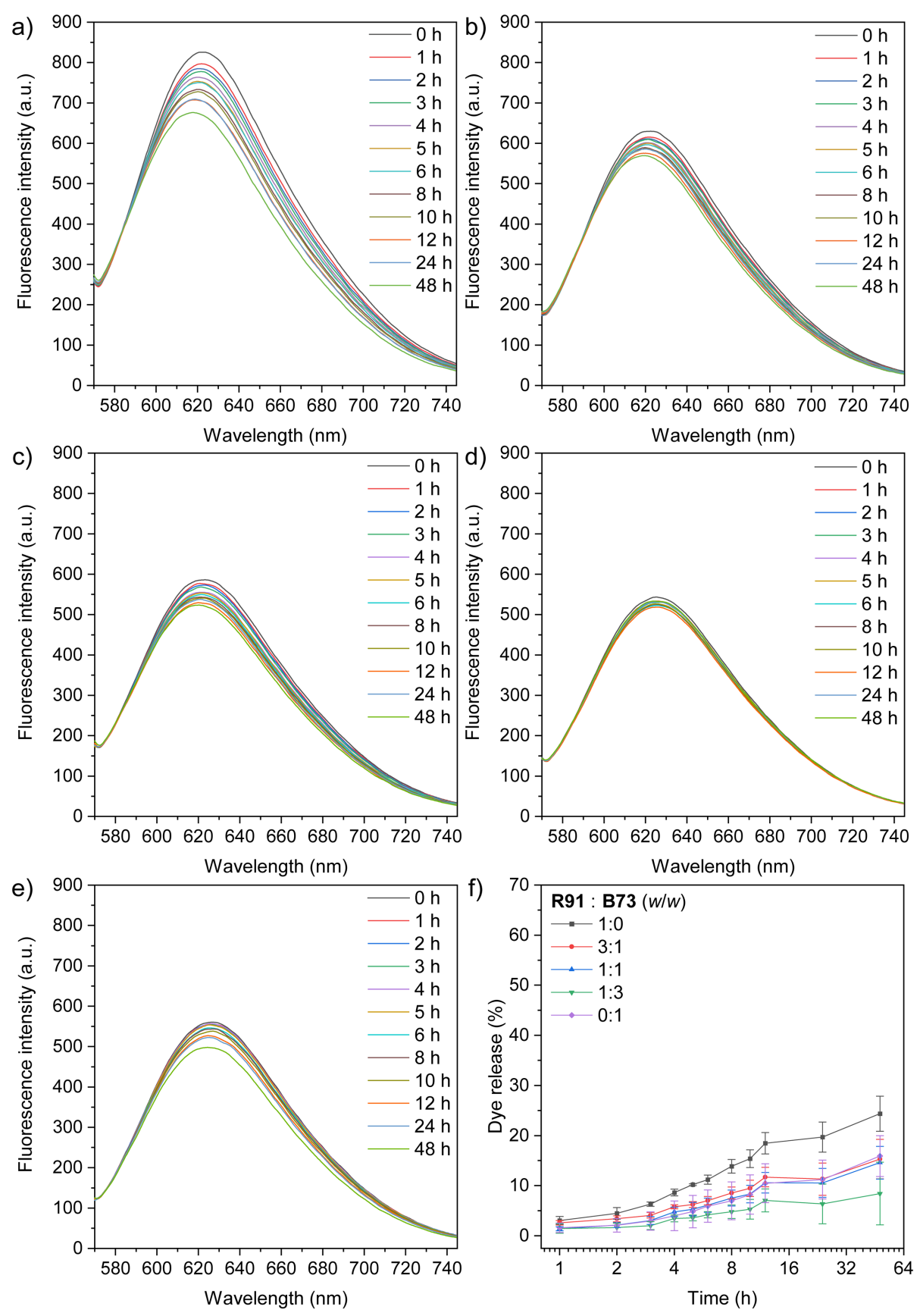

Figure S21. Representative time-dependent fluorescence emission spectra of Nile red within the assemblies of (a) R91, (b) R91 and B73 (w/w = 3:1), (c) R91 and B73 (w/w = 1:1), (d) R91 and B73 (w/w $=1: 3$ ), and (e) B73 without the presence of glutathione. The copolymers in each group were treated with 10 mol\% (vs. PDS units) of DTT to aim at 20 mol\% crosslinking of the PDS units. (f) Cargo (Nile red) release profiles of the self-assemblies formed by individual or blended copolymers without the addition of glutathione. 

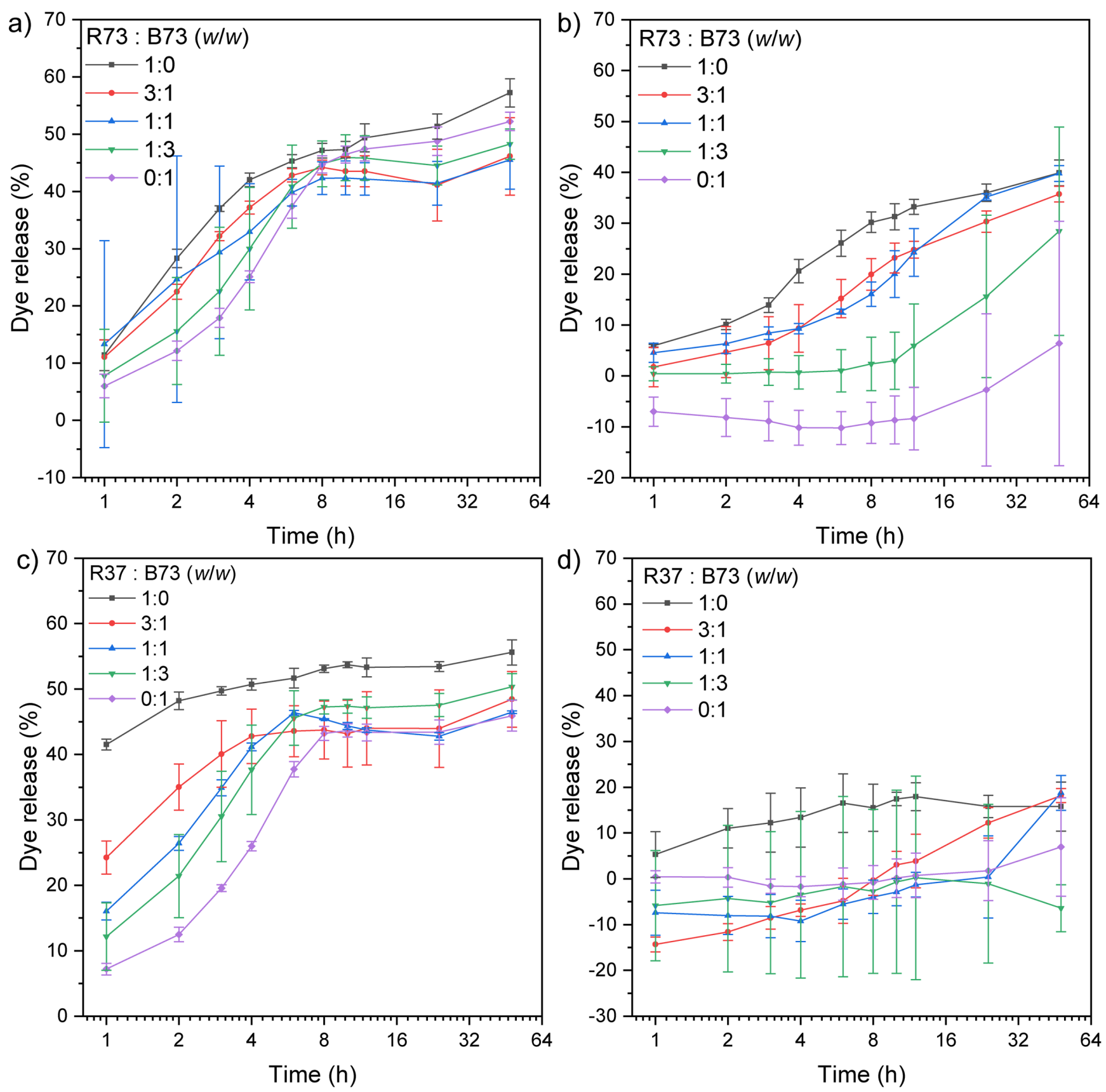

Figure S22. Glutathione-triggered cargo (Nile red) release profiles of the self-assemblies formed by individual or blended copolymers with different degree of crosslinking. The copolymers in each group were treated with $(a, c) 2.5 \mathrm{~mol} \%$ (vs. PDS units) of DTT to aim at 5 mol\% crosslinking, $(b, d) 25$ mol\% (vs. PDS units) of DTT to aim at 50 mol\% crosslinking of the PDS units. Glutathione was maintained at a final concentration of $10 \mathrm{mM}$ to trigger the Nile red release. In each figure, error bars represent the standard deviation of three replicates. 

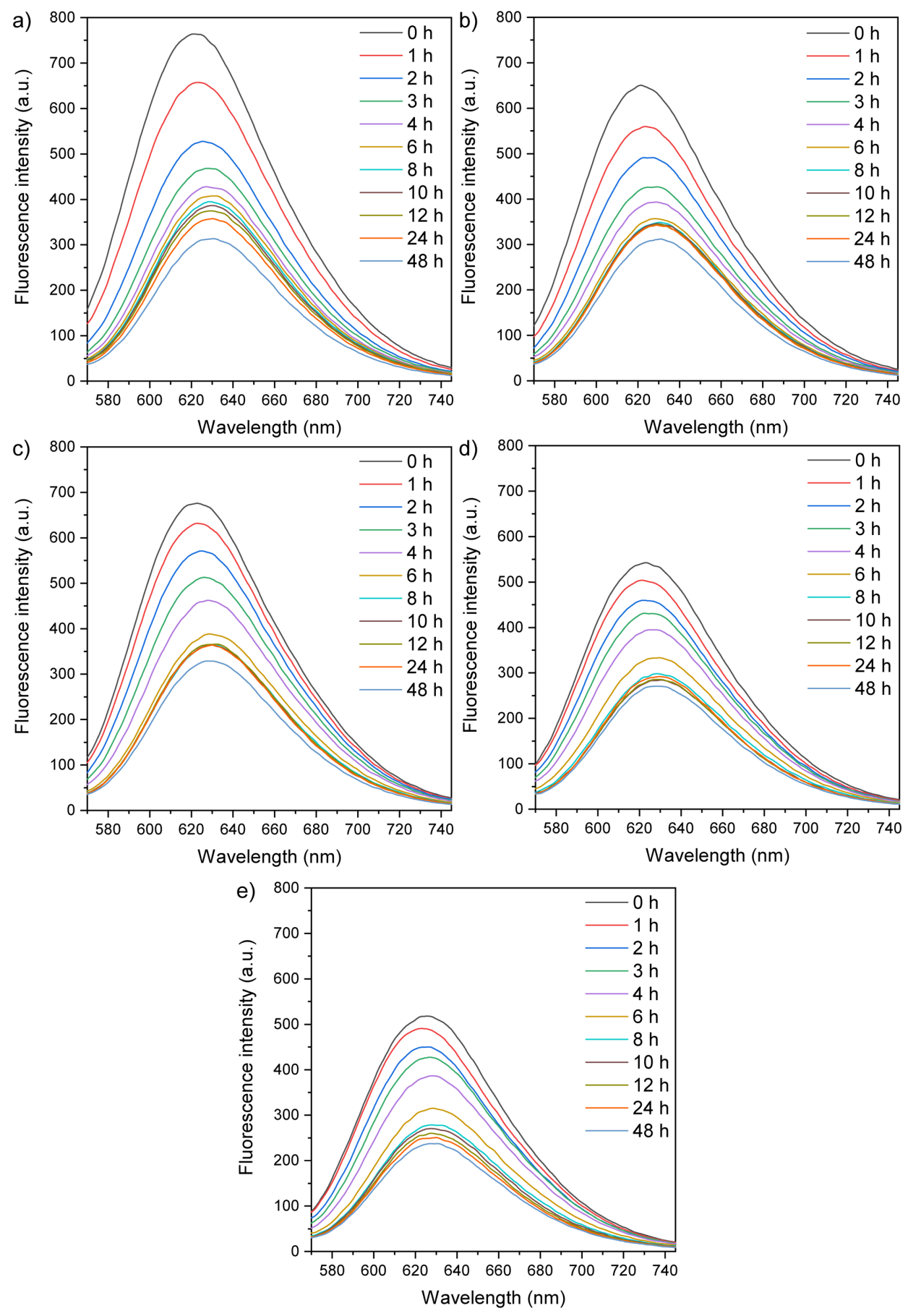

Figure S23. Representative time-dependent fluorescence emission spectra of Nile red within the assemblies of (a) R73, (b) R73 and B73 ( $w / w=3: 1$ ), (c) R73 and B73 (w/w = 1:1), (d) R73 and B73 (w/w $=1: 3$ ), and (e) B73. The copolymers in each group were treated with $2.5 \mathrm{~mol} \%$ (vs. PDS units) of DTT to aim at $5 \mathrm{~mol} \%$ crosslinking of the PDS units. Glutathione was maintained at a final concentration of 10 $\mathrm{mM}$ to trigger the Nile red release. 

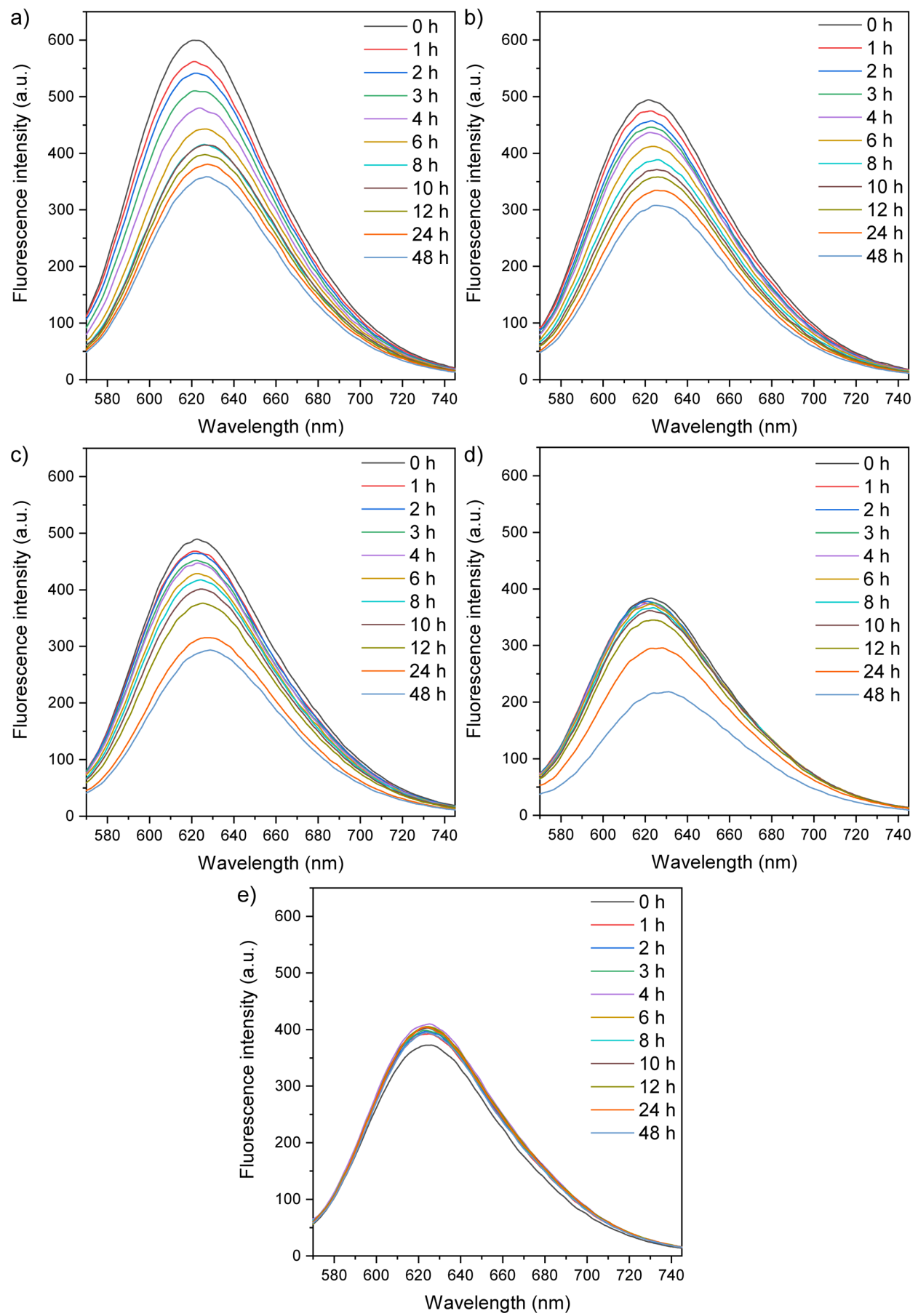

Figure S24. Representative time-dependent fluorescence emission spectra of Nile red within the assemblies of (a) R73, (b) R73 and B73 ( $w / w=3: 1$ ), (c) R73 and B73 (w/w = 1:1), (d) R73 and B73 (w/w $=1: 3$ ), and (e) B73. The copolymers in each group were treated with 25 mol\% (vs. PDS units) of DTT to aim at $50 \mathrm{~mol} \%$ crosslinking of the PDS units. Glutathione was maintained at a final concentration of 10 $\mathrm{mM}$ to trigger the Nile red release. 

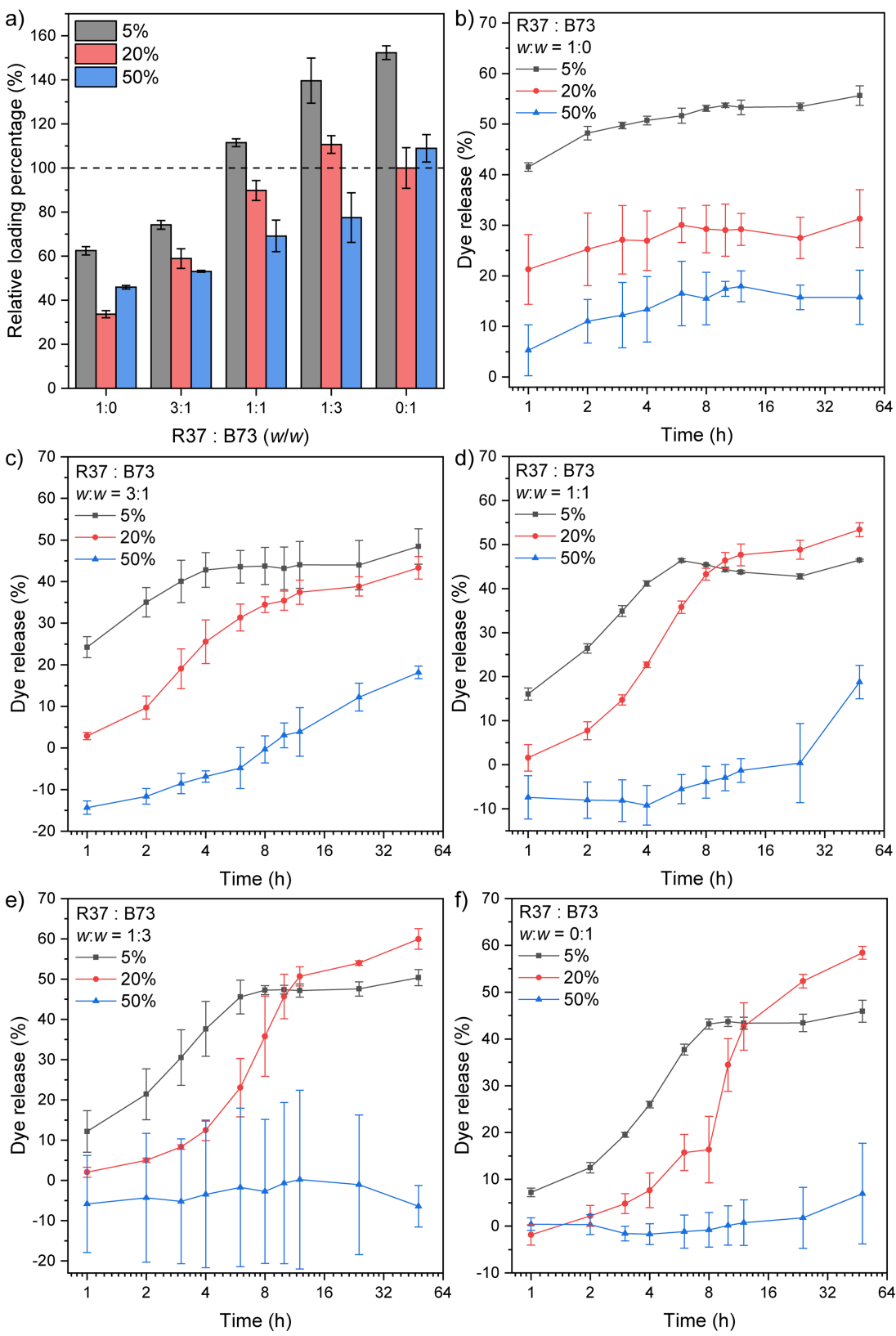

Figure S25. (a) Cargo (Nile red) encapsulation and (b f) glutathione-triggered cargo release profiles of the self-assemblies of R37-B73 blended copolymers with varied crosslinking degree. The copolymers in each group were treated with $2.5 \mathrm{~mol} \%, 10 \mathrm{~mol} \%$, or $25 \mathrm{~mol} \%$ (vs. PDS units) of DTT to aim at $5 \mathrm{~mol} \%$, $20 \mathrm{~mol} \%$, or $50 \mathrm{~mol} \%$ crosslinking of the PDS units, respectively. The relative loading percentage of selfassembled B73 with $20 \mathrm{~mol} \%$ crosslinking is denoted with a dashed line in (a) as a reference. Glutathione was maintained at a final concentration of $10 \mathrm{mM}$ to trigger the Nile red release. In each figure, error bars represent the standard deviation of three replicates. 

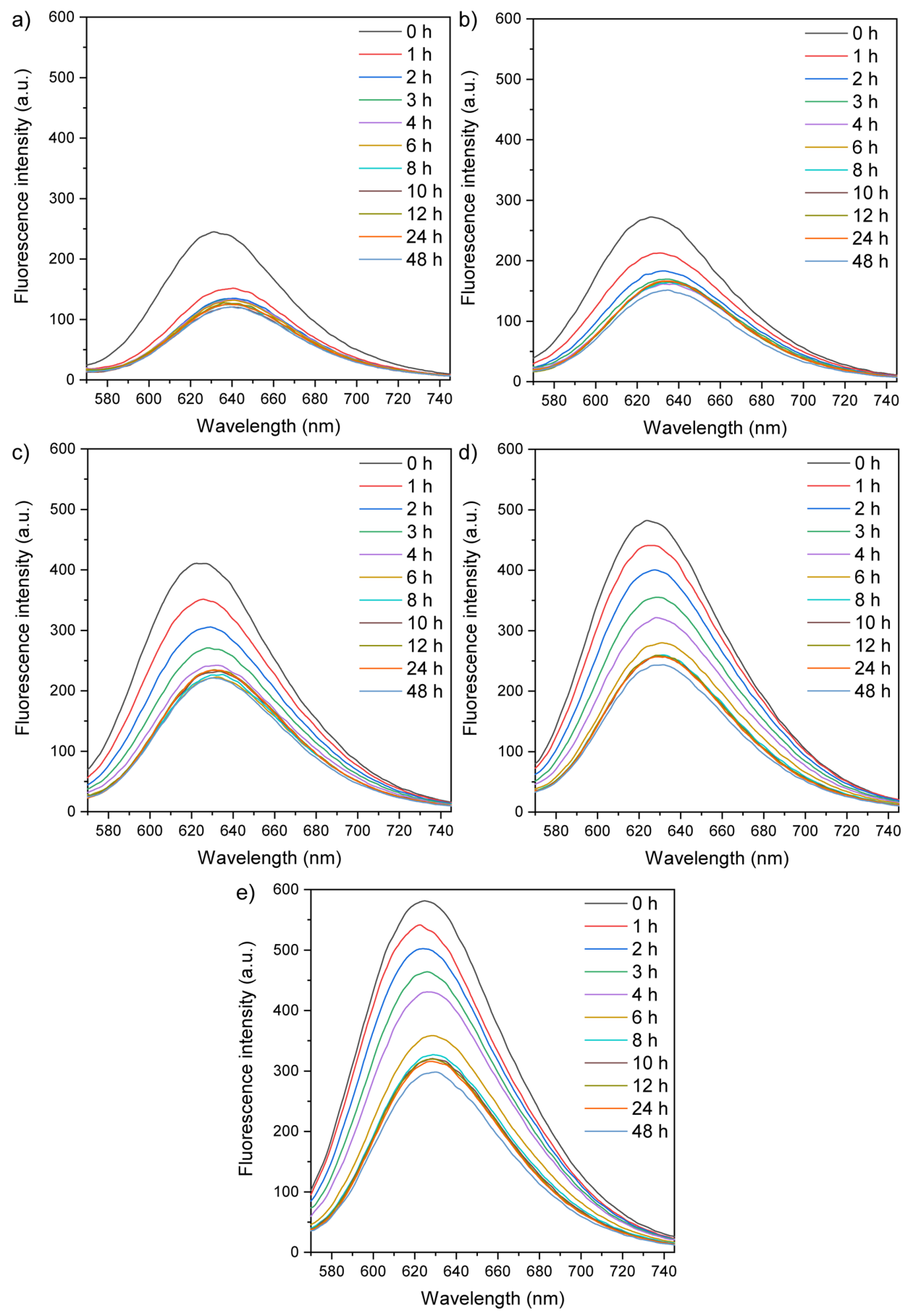

Figure S26. Representative time-dependent fluorescence emission spectra of Nile red within the assemblies of (a) R37, (b) R37 and B73 (w/w = 3:1), (c) R37 and B73 (w/w = 1:1), (d) R37 and B73 (w/w $=1: 3$ ), and (e) B73. The copolymers in each group were treated with $2.5 \mathrm{~mol} \%$ (vs. PDS units) of DTT to aim at $5 \mathrm{~mol} \%$ crosslinking of the PDS units. Glutathione was maintained at a final concentration of 10 $\mathrm{mM}$ to trigger the Nile red release. 

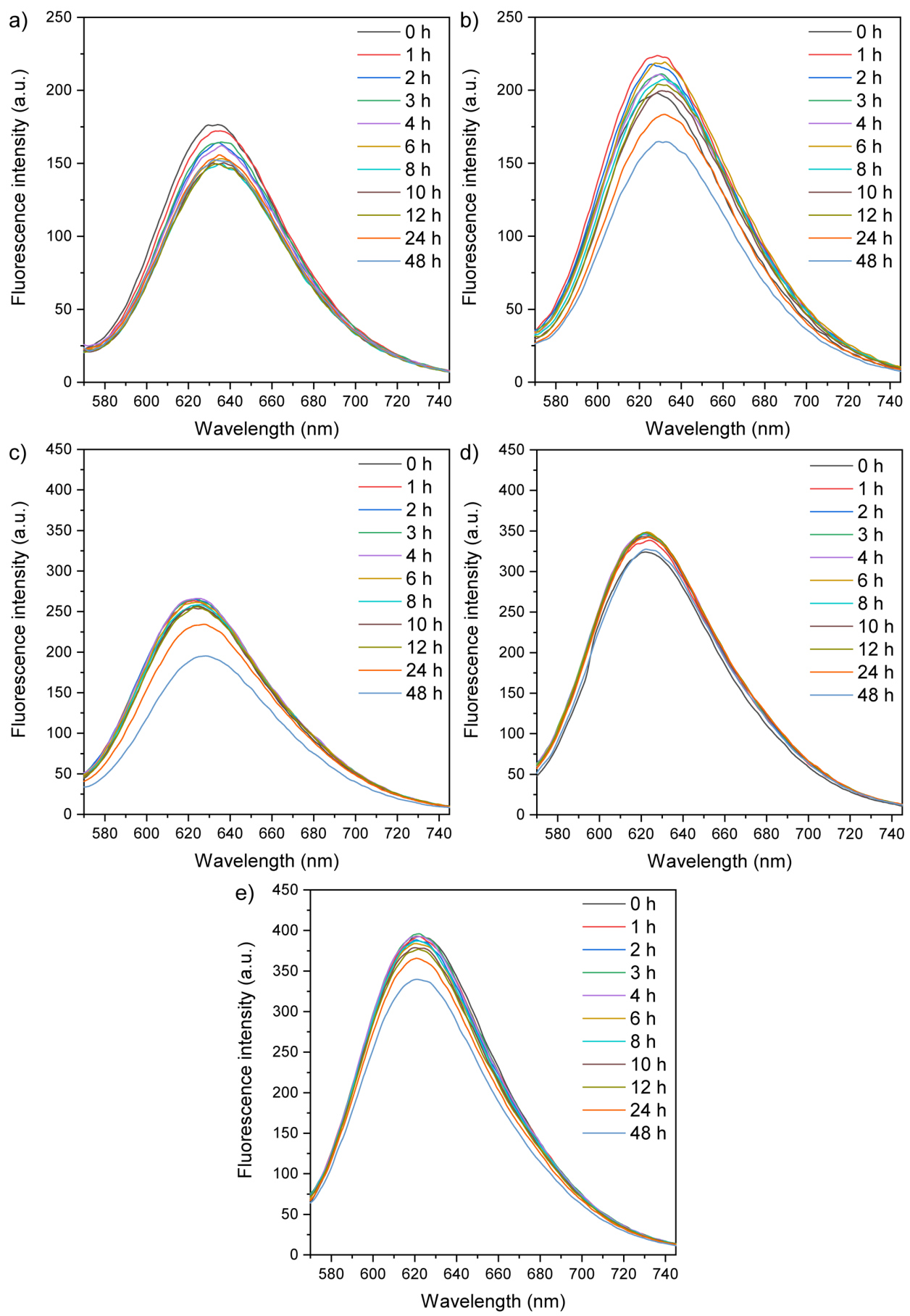

Figure S27. Representative time-dependent fluorescence emission spectra of Nile red within the assemblies of (a) R37, (b) R37 and B73 ( $w / w=3: 1$ ), (c) R37 and B73 ( $w / w=1: 1$ ), (d) R37 and B73 ( $w / w$ = 1:3), and (e) B73. The copolymers in each group were treated with 25 mol\% (vs. PDS units) of DTT to aim at $50 \mathrm{~mol} \%$ crosslinking of the PDS units. Glutathione was maintained at a final concentration of 10 $\mathrm{mM}$ to trigger the Nile red release. 

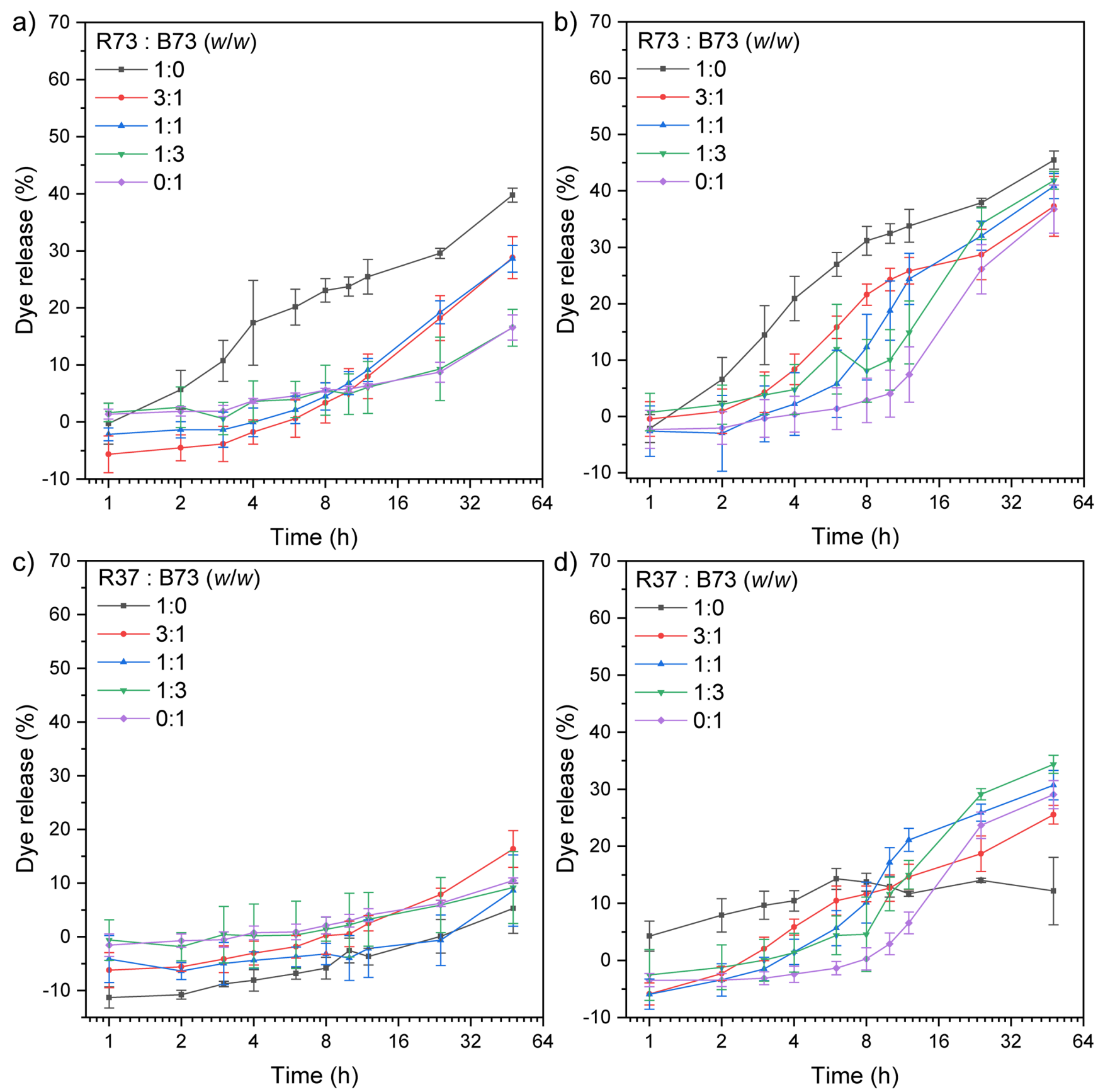

Figure S28. Glutathione-triggered cargo (Nile red) release profiles of the self-assemblies formed by individual or blended copolymers with different disulfide-containing side chains. The copolymers in each group with 20\% DTT-induced crosslinking were treated with either $(a, c)$ PEM or $(b, d)$ TriEG to replace the rest of the unreacted PDS units within the assemblies, followed by glutathione-treatment. Glutathione was maintained at a final concentration of $10 \mathrm{mM}$ to trigger the Nile red release. In each figure, error bars represent the standard deviation of three replicates. 

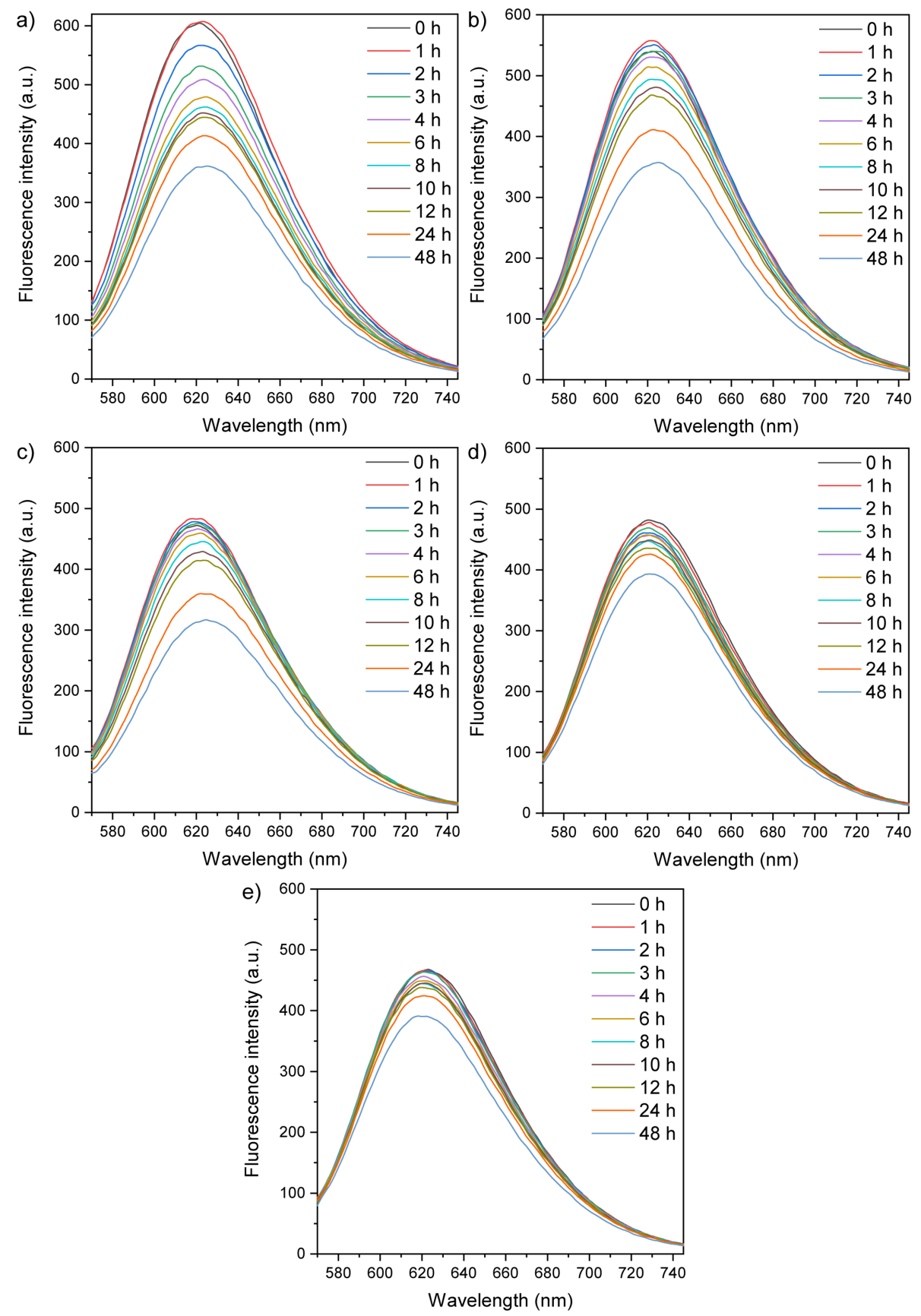

Figure S29. Representative time-dependent fluorescence emission spectra of Nile red within the assemblies of (a) R73, (b) R73 and B73 ( $w / w=3: 1$ ), (c) R73 and B73 ( $w / w=1: 1$ ), (d) R73 and B73 (w/w = 1:3), and (e) B73. The copolymers in each group were treated with $10 \mathrm{~mol} \%$ (vs. PDS units) of DTT to aim at 20 mol\% crosslinking of the PDS units, followed by the treatment of 2-pyridylethylmercaptan (PEM) to replace the rest of the PDS units. Glutathione was maintained at a final concentration of $10 \mathrm{mM}$ to trigger the Nile red release. 

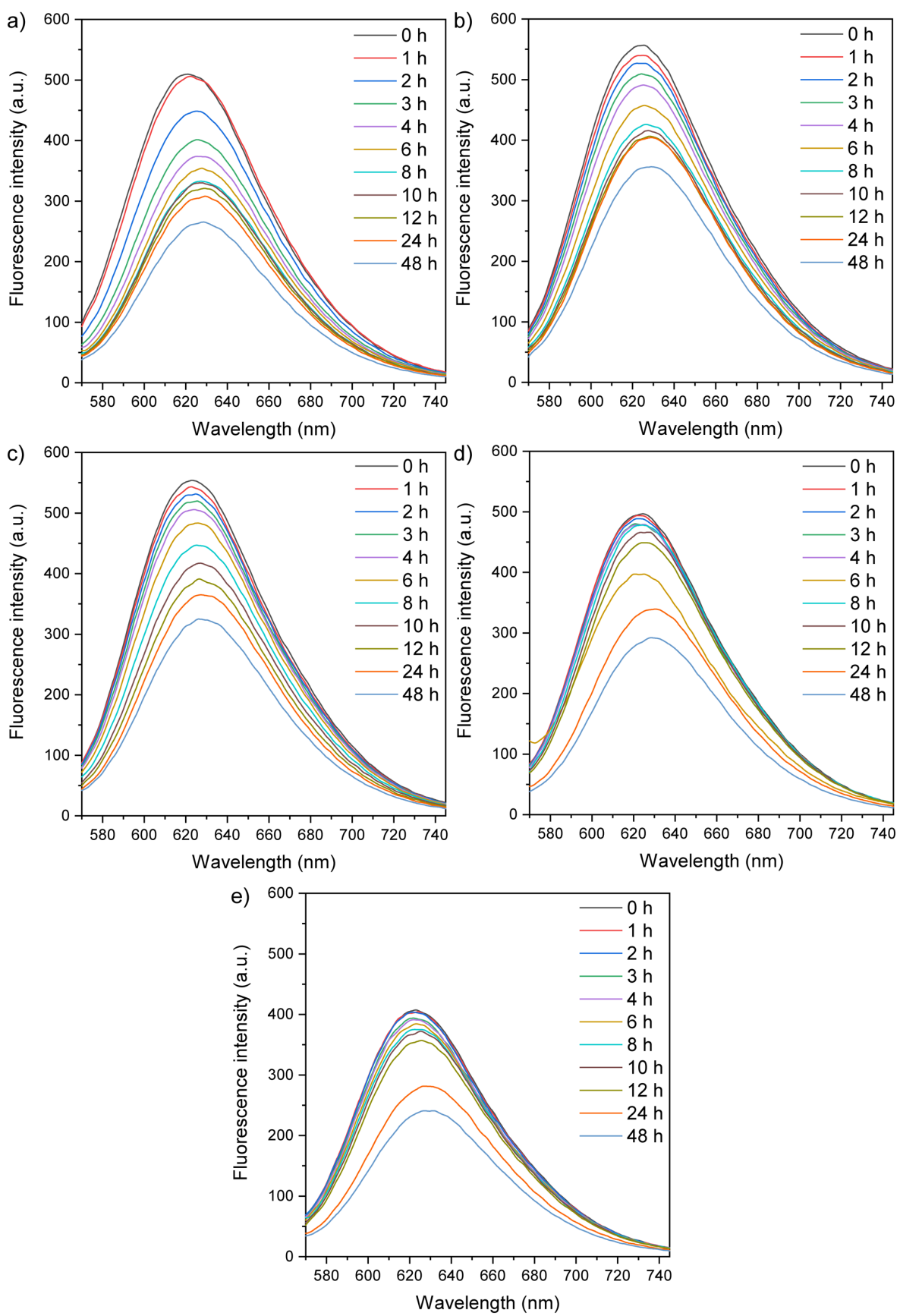

Figure S30. Representative time-dependent fluorescence emission spectra of Nile red within the assemblies of (a) R73, (b) R73 and B73 ( $w / w=3: 1$ ), (c) R73 and B73 (w/w = 1:1), (d) R73 and B73 ( $w / w$ $=1: 3$ ), and (e) B73. The copolymers in each group were treated with 10 mol\% (vs. PDS units) of DTT to aim at 20 mol\% crosslinking of the PDS units, followed by the treatment of 3,6-dioxa-8-mercaptooctan1-ol (TriEG) to replace the rest of the PDS units. Glutathione was maintained at a final concentration of $10 \mathrm{mM}$ to trigger the Nile red release. 

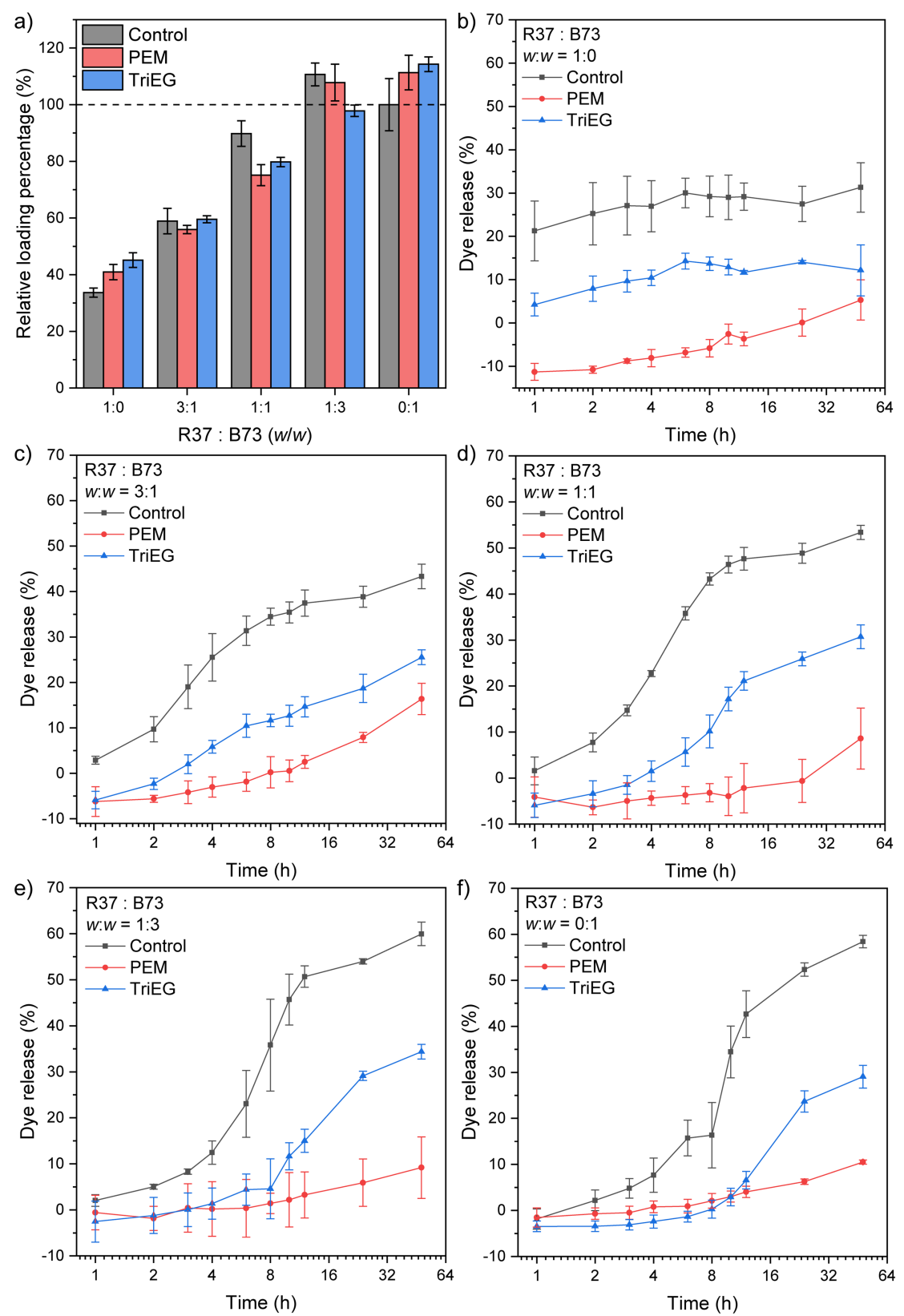

Figure S31. (a) Cargo (Nile red) encapsulation and (b f) glutathione-triggered cargo release profiles of the self-assemblies of R37-B73 blended copolymers with varied disulfide-containing side chains. The copolymers in each group with $20 \%$ DTT-induced crosslinking were treated with either PEM or TriEG (no such treatment for the control group) to replace the rest of the unreacted PDS units within the assemblies. The relative loading percentage of $20 \%$-crosslinked B73 assemblies without thiol replacement is denoted with a dashed line in (a) as a reference. Glutathione was maintained at a final concentration of $10 \mathrm{mM}$ to trigger the Nile red release. In each figure, error bars represent the standard deviation of three replicates. 

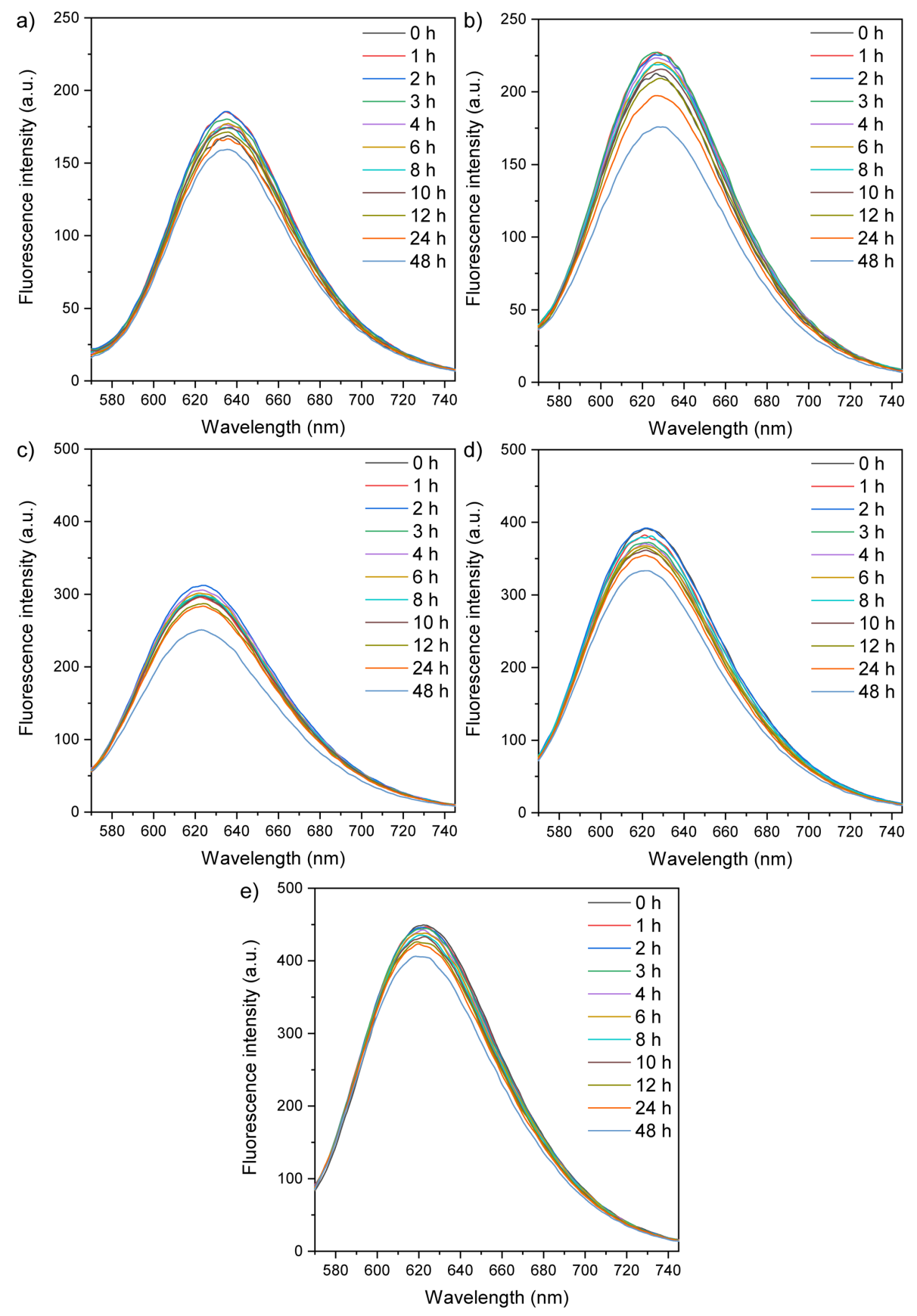

Figure S32. Representative time-dependent fluorescence emission spectra of Nile red within the assemblies of (a) R37, (b) R37 and B73 (w/w = 3:1), (c) R37 and B73 ( $w / w=1: 1$ ), (d) R37 and B73 ( $w / w$ $=1: 3$ ), and (e) B73. The copolymers in each group were treated with 10 mol\% (vs. PDS units) of DTT to aim at 20 mol\% crosslinking of the PDS units, followed by the treatment of 2-pyridylethylmercaptan (PEM) to replace the rest of the PDS units. Glutathione was maintained at a final concentration of $10 \mathrm{mM}$ to trigger the Nile red release. 

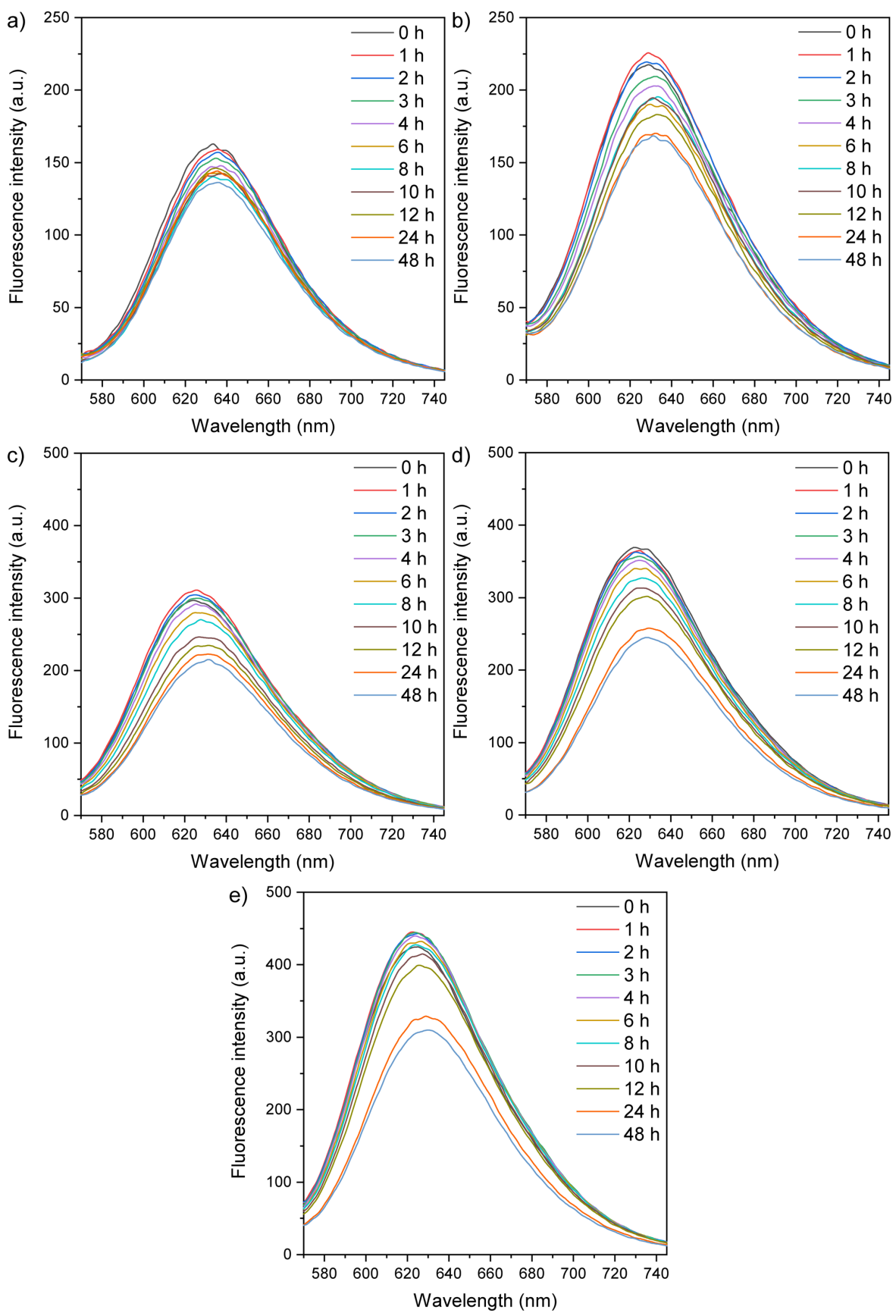

Figure S33. Representative time-dependent fluorescence emission spectra of Nile red within the assemblies of (a) R37, (b) R37 and B73 ( $w / w=3: 1$ ), (c) R37 and B73 (w/w = 1:1), (d) R37 and B73 ( $w / w$ $=1: 3$ ), and (e) B73. The copolymers in each group were treated with $10 \mathrm{~mol} \%$ (vs. PDS units) of DTT to aim at 20 mol\% crosslinking of the PDS units, followed by the treatment of 3,6-dioxa-8-mercaptooctan1-ol (TriEG) to replace the rest of the PDS units. Glutathione was maintained at a final concentration of $10 \mathrm{mM}$ to trigger the Nile red release. 

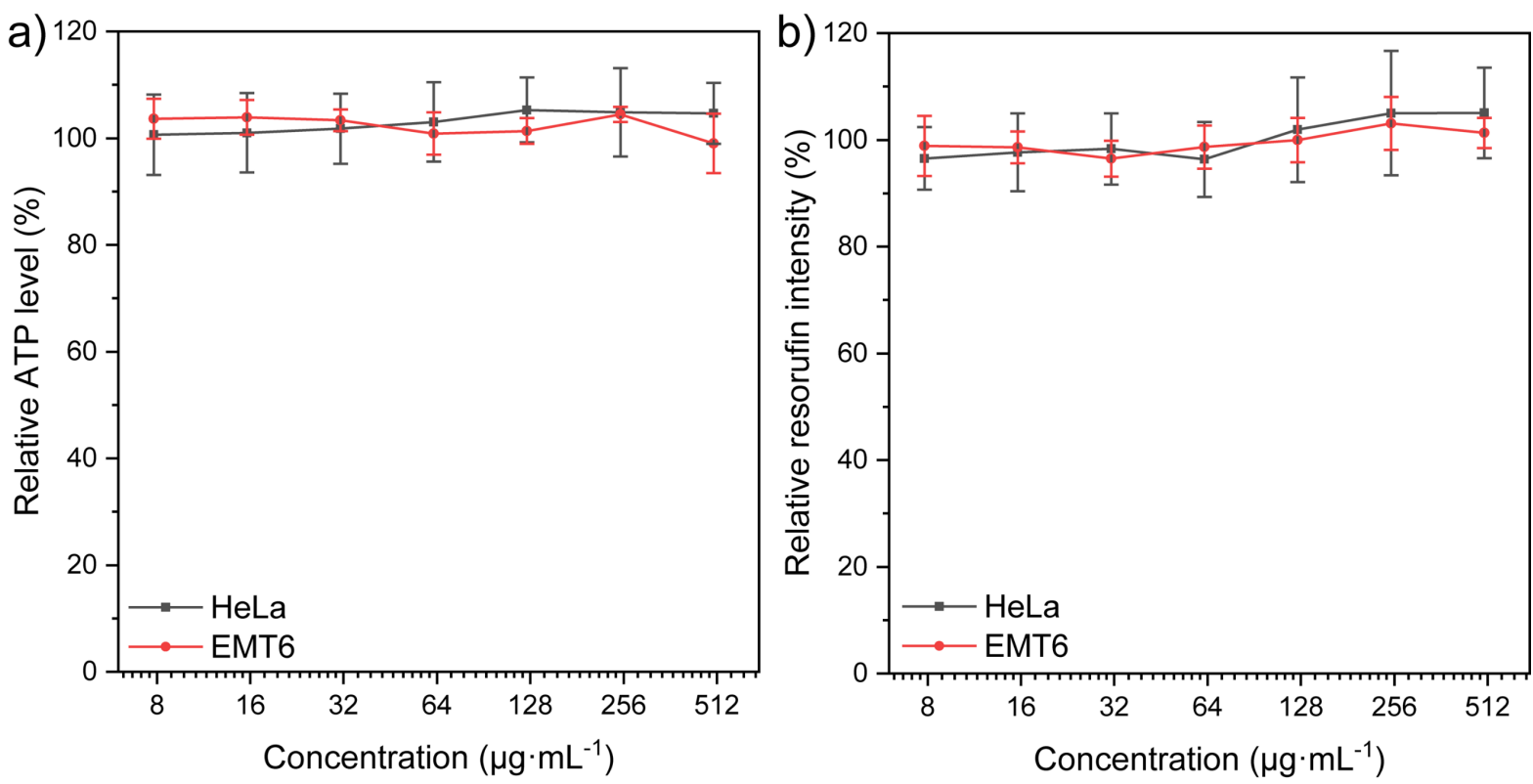

Figure 34. (a) Relative ATP level and (b) metabolic activity of mammalian cells after incubating with R73B73 co-assemblies at different concentrations for 24 hours. The metabolic activity was quantified by alamarBlue assay. In each figure, error bars represent the standard deviation of four replicates. We chose the 1:1 (w/w) combination between $\mathbf{R 7 3}$ and B73 to formulate the co-assemblies for further assessment. After incubating the co-assemblies with either human cervical cancer cells (HeLa) or murine mammary carcinoma cells (EMT6) for 24 hours, the ATP production level and metabolic activity of each cell line were not interfered up to a concentration of $0.5 \mathrm{mg} \cdot \mathrm{mL}^{-1}$ for the co-assemblies. These results demonstrated the wide biocompatible range of the R73-B73 co-assemblies. Understanding the biocompatibility profiles of each polymer combination will indeed build a comprehensive reference for drug delivery applications of the co-assemblies. As the focus of the current study is on the cargo loading and release properties of the co-assemblies, identifying a general biocompatible range will aid the future investigations on using these co-assemblies for therapeutic delivery. 


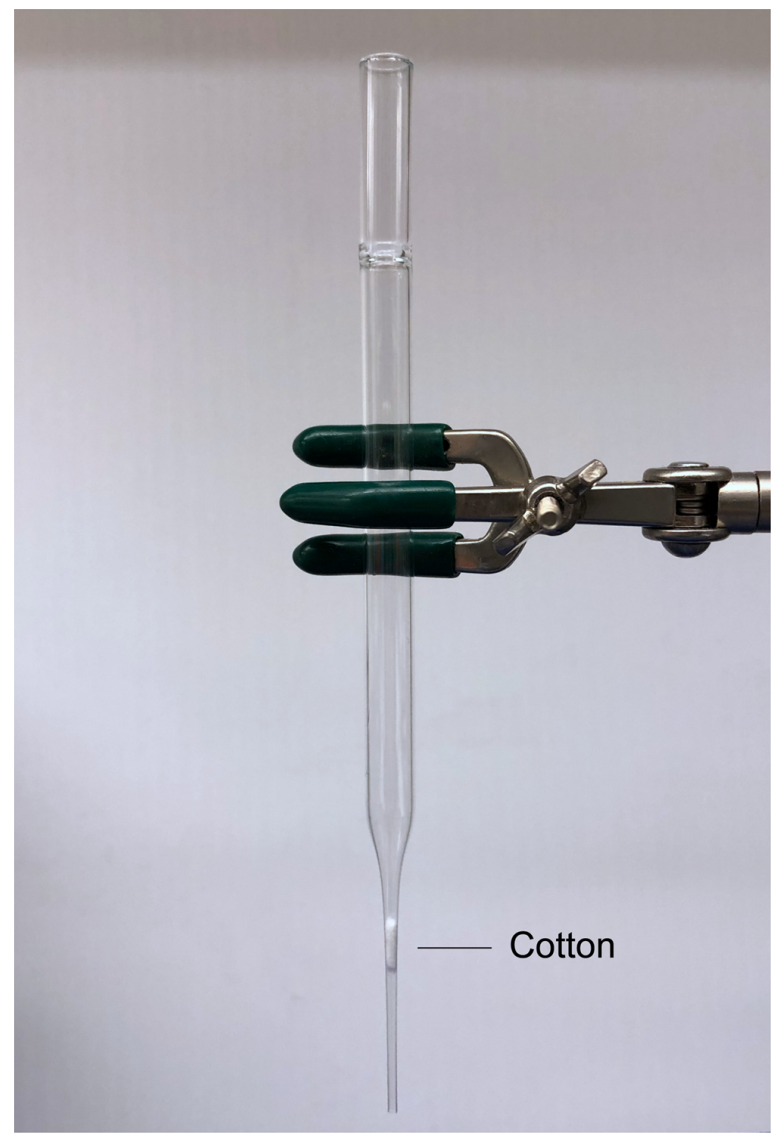

Figure S35. Photograph of cotton-plugged glass Pasteur pipette (Fisher Scientific, Cat\# 22-183624) for the filtration of un-encapsulated hydrophobic cargo molecules. 


\section{Experimental Section}

Table S2. Key regents in the study.

\begin{tabular}{|c|c|c|}
\hline Reagent & Source & Identifier \\
\hline $\begin{array}{l}\text { Poly(ethylene glycol) methyl ether } \\
\text { methacrylate }\left(M_{\mathrm{n}} \sim 500\right)\end{array}$ & Sigma-Aldrich & Cat\# 447943 \\
\hline 2,2'-Dithiodipyridine & Chem-Impex International & Cat\# 00124 \\
\hline 2-Mercaptoethanol & Sigma-Aldrich & Cat\# M6250 \\
\hline Methacryloyl chloride & Alfa Aesar & Cat\# L14511 \\
\hline 2,2'-Azobis(2-methylpropionitrile) & Sigma-Aldrich & Cat\# 441090 \\
\hline $\begin{array}{l}\text { 4-Cyano-4- } \\
\text { (phenylcarbonothioylthio)pentanoic } \\
\text { acid }\end{array}$ & Sigma-Aldrich & Cat\# 722995 \\
\hline $\begin{array}{l}\text { Methoxy PEG Amine, } \mathrm{HCl} \text { Salt }\left(M_{\mathrm{n}}\right. \\
\sim 5000)\end{array}$ & JenKem Technology & Cat\# A3035-1/M-NH2HCl-5000 \\
\hline $\begin{array}{l}\text { 4-Cyano-4- } \\
\text { (phenylcarbonothioylthio)pentanoic } \\
\text { acid } N \text {-succinimidyl ester }\end{array}$ & Sigma-Aldrich & Cat\# 758353 \\
\hline 4-Bromobenzyl mercaptan & Sigma-Aldrich & Cat\# 566950 \\
\hline 6-(Ferrocenyl)hexanethiol & AstaTech & Cat\# TD8104 \\
\hline DBCO-Cy3 & Lumiprobe & Cat\# C10F0 \\
\hline DBCO-Cy5 & Lumiprobe & Cat\# C30F0 \\
\hline Nile red & Chem-Impex International & Cat\# 22855 \\
\hline Sudan red G & Sigma-Aldrich & Cat\# 17173 \\
\hline Sudan black B & Sigma-Aldrich & Cat\# 199664 \\
\hline Oil red $\mathrm{O}$ & Sigma-Aldrich & Cat\# O0625 \\
\hline Dithiothreitol & Fisher Scientific & Cat\# BP172-5 \\
\hline Glutathione, reduced & Fisher Scientific & Cat\# BP25215 \\
\hline SorbaDex gel filtration matrix, 20-LH & Sorbtech & Cat\# 801009 \\
\hline $\begin{array}{l}\text { Polyethylene glycol standard, nominal } \\
M_{\mathrm{p}} 10,000 \mathrm{~g} \cdot \mathrm{mol}^{-1}\end{array}$ & Agilent & Cat\# PL2070-9001 \\
\hline
\end{tabular}




\subsection{Polymer synthesis}
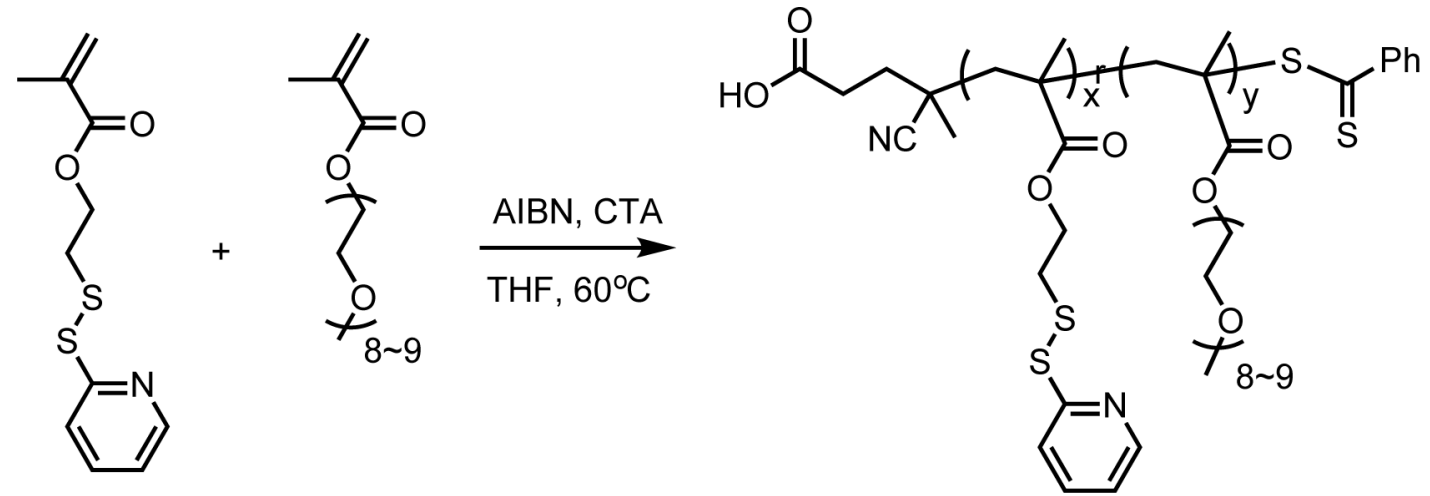

R37, an amphiphilic random copolymer. Pyridyl disulfide ethyl methacrylate $(178 \mathrm{mg}, 0.70 \mathrm{mmol}, 7.0$ equiv.), poly(ethylene glycol) methyl ether methacrylate (820 mg, 1.64 mmol, 16.4 equiv., $\left.M_{\mathrm{n}} 500\right)$, 4cyano-4-(phenylcarbonothioylthio)pentanoic acid (CTA, $27.9 \mathrm{mg}, 0.1 \mathrm{mmol}, 1.0$ equiv.), and AIBN (3.3 $\mathrm{mg}, 0.02 \mathrm{mmol}, 0.2$ equiv.) were dissolved in $2.1 \mathrm{~mL}$ anhydrous tetrahydrofuran (THF) and transferred to a $10-\mathrm{mL}$ Schlenk flask. The flask was sealed and the reaction mixture was degassed with four freezepump-thaw cycles. After refilling the flask with an argon atmosphere, the reaction was dipped into a prewarmed oil bath at $60{ }^{\circ} \mathrm{C}$ and stirred for 20 hours. Next, the polymerization was quenched by freezing the mixture with liquid nitrogen and exposing to air. The polymer was purified by dissolving in $\mathrm{CHCl}_{3}$ and subsequently precipitating in cold diethyl ether for three times. The product was dried under vacuum and obtained as viscous pink oil (861 mg). GPC (THF), $M_{\mathrm{n}}: 12.8 \mathrm{~kg} \cdot \mathrm{mol}^{-1}, Ð: 1.15 .{ }^{1} \mathrm{H} \mathrm{NMR}\left(400 \mathrm{MHz}, \mathrm{CDCl}_{3}\right)$ $\delta 8.45$ (s), 7.69 (s), 7.13 (s), 4.22 (s), 4.07 (s), 3.71 - $3.64(\mathrm{~m}), 3.56$ (s), 3.38 (s), 3.04 (s), 1.80 (s), 1.69 (s), 1.03 (s), 0.86 (s) (Figure SP1). The molar ratio between two repeating units was determined by integrating the aromatic proton in the pyridine and the methoxy proton $(\delta 3.3)$ in the polyethylene glycol side chain $(\mathrm{x}: \mathrm{y}=0.29: 0.71) .{ }^{13} \mathrm{C}$ NMR $\left(125 \mathrm{MHz}_{\mathrm{CDCl}}\right.$ ) (Figure SP2). IR spectrum and TGA curve (Figure SP3). 
R55, an amphiphilic random copolymer. Pyridyl disulfide ethyl methacrylate (500 mg, 1.96 mmol, 13.2 equiv.), poly(ethylene glycol) methyl ether methacrylate (979 mg, 1.96 mmol, 13.2 equiv., $M_{\mathrm{n}}$ 500), CTA (41.3 mg, $0.15 \mathrm{mmol}, 1.0$ equiv.), and AIBN (4.9 mg, $0.03 \mathrm{mmol}, 0.2$ equiv.) were dissolved in $3 \mathrm{~mL}$ anhydrous tetrahydrofuran (THF) and transferred to a $10-\mathrm{mL}$ Schlenk flask. The flask was sealed and the reaction mixture was degassed with four freeze-pump-thaw cycles. After refilling the flask with an argon atmosphere, the reaction was dipped into a prewarmed oil bath at $60^{\circ} \mathrm{C}$ and stirred for 20 hours. Next, the polymerization was quenched by freezing the mixture with liquid nitrogen and exposing to air. The polymer was purified by dissolving in $\mathrm{CHCl}_{3}$ and subsequently precipitating in cold diethyl ether for three times. The product was dried under vacuum and obtained as viscous pink oil (1.48 g). GPC (THF), $M_{\mathrm{n}}: 11.3 \mathrm{k} \mathrm{g} \cdot \mathrm{mol}^{-1}, \oplus: 1.11 .{ }^{1} \mathrm{H}$ NMR $\left(500 \mathrm{MHz} \mathrm{CDCl}_{3}\right) \delta 8.46$ (s), 7.68 (s), 7.11 (s), 4.21 (s), 4.07 (s), $3.71-3.64(\mathrm{~m}), 3.54(\mathrm{~s}), 3.37(\mathrm{~s}), 3.02(\mathrm{~s}), 1.90(\mathrm{~s}), 1.81(\mathrm{~s}), 1.06(\mathrm{~s}), 0.88$ (s) (Figure SP4). The molar ratio between two repeating units was determined by integrating the aromatic proton in the pyridine and the methoxy proton $(\delta 3.3)$ in the polyethylene glycol side chain $(x: y=0.49: 0.51) .{ }^{13} \mathrm{C} N M R(125 \mathrm{MHz}$, $\left.\mathrm{CDCl}_{3}\right)$ (Figure SP5). IR spectrum and TGA curve (Figure SP6).

R73, an amphiphilic random copolymer. Pyridyl disulfide ethyl methacrylate $(600 \mathrm{mg}, 2.35 \mathrm{mmol}, 21.0$ equiv.), poly(ethylene glycol) methyl ether methacrylate (392 mg, $0.78 \mathrm{mmol}, 9.0$ equiv., $M_{\mathrm{n}} 500$ ), CTA (31.3 mg, $0.11 \mathrm{mmol}, 1.0$ equiv.), and AIBN (3.7 mg, $0.02 \mathrm{mmol}, 0.2$ equiv.) were dissolved in $2 \mathrm{~mL}$ anhydrous tetrahydrofuran (THF) and transferred to a $10-\mathrm{mL}$ Schlenk flask. The flask was sealed and the reaction mixture was degassed with four freeze-pump-thaw cycles. After refilling the flask with an argon atmosphere, the reaction was dipped into a prewarmed oil bath at $60{ }^{\circ} \mathrm{C}$ and stirred for 20 hours. Next, the polymerization was quenched by freezing the mixture with liquid nitrogen and exposing to air. The polymer was purified by dissolving in $\mathrm{CHCl}_{3}$ and subsequently precipitating in cold diethyl ether for three times. The product was dried under vacuum and obtained as viscous pink oil (792 mg). GPC (THF), $M_{\mathrm{n}}: 12.3 \mathrm{k} \mathrm{g} \cdot \mathrm{mol}^{-1}, \oplus: 1.18 .{ }^{1} \mathrm{H}$ NMR (400 MHz, $\left.\mathrm{CDCl}_{3}\right) \delta 8.45$ (s), 7.66 (s), 7.10 (s), 4.21 (s), 4.07 (s), 
3.63 (s), 3.54 (s), 3.37 (s), 3.02 (s), 1.90 (s), 1.83 (s), 1.06 (s), 0.89 (s) (Figure SP7). The molar ratio between two repeating units was determined by integrating the aromatic proton in the pyridine and the methoxy proton ( $\delta 3.3)$ in the polyethylene glycol side chain $(x: y=0.70: 0.30) .{ }^{13} \mathrm{C} \mathrm{NMR}\left(125 \mathrm{MHz}, \mathrm{CDCl}_{3}\right)$ (Figure SP8). IR spectrum and TGA curve (Figure SP9).

R91, an amphiphilic random copolymer. Pyridyl disulfide ethyl methacrylate $(800 \mathrm{mg}, 3.13 \mathrm{mmol}, 32.2$ equiv.), poly(ethylene glycol) methyl ether methacrylate (174 mg, $0.35 \mathrm{mmol}, 3.6$ equiv., $M_{\mathrm{n}} 500$ ), CTA (27.2 mg, $0.10 \mathrm{mmol}, 1.0$ equiv.), and AIBN (3.2 mg, $0.02 \mathrm{mmol}, 0.2$ equiv.) were dissolved in $2 \mathrm{~mL}$ anhydrous tetrahydrofuran (THF) and transferred to a 10-mL Schlenk flask. The flask was sealed and the reaction mixture was degassed with four freeze-pump-thaw cycles. After refilling the flask with an argon atmosphere, the reaction was dipped into a prewarmed oil bath at $60^{\circ} \mathrm{C}$ and stirred for 20 hours. Next, the polymerization was quenched by freezing the mixture with liquid nitrogen and exposing to air. The polymer was purified by dissolving in $\mathrm{CHCl}_{3}$ and subsequently precipitating in cold diethyl ether for three times. The product was dried under vacuum and obtained as viscous pink oil (816 mg). GPC (THF), $M_{\mathrm{n}}: 9.2 \mathrm{k} \mathrm{g} \cdot \mathrm{mol}^{-1}$,, : 1.19. ${ }^{1} \mathrm{H} \mathrm{NMR}\left(400 \mathrm{MHz}, \mathrm{CDCl}_{3}\right) \delta 8.45$ (s), 7.65 (s), 7.09 (s), 4.21 (s), 4.07 (s), 3.64 (s), $3.54(\mathrm{~s}), 3.37(\mathrm{~s}), 3.02(\mathrm{~s}), 1.84(\mathrm{~s}), 1.59(\mathrm{~s}), 1.06$ (s), 0.90 (s) (Figure SP10). The molar ratio between two repeating units was determined by integrating the aromatic proton in the pyridine and the methoxy proton $(\delta 3.3)$ in the polyethylene glycol side chain $(\mathrm{x}: \mathrm{y}=0.89: 0.11) \cdot{ }^{13} \mathrm{C} \mathrm{NMR}\left(125 \mathrm{MHz}, \mathrm{CDCl}_{3}\right)(\mathrm{Figure}$ SP11). IR spectrum and TGA curve (Figure SP12).

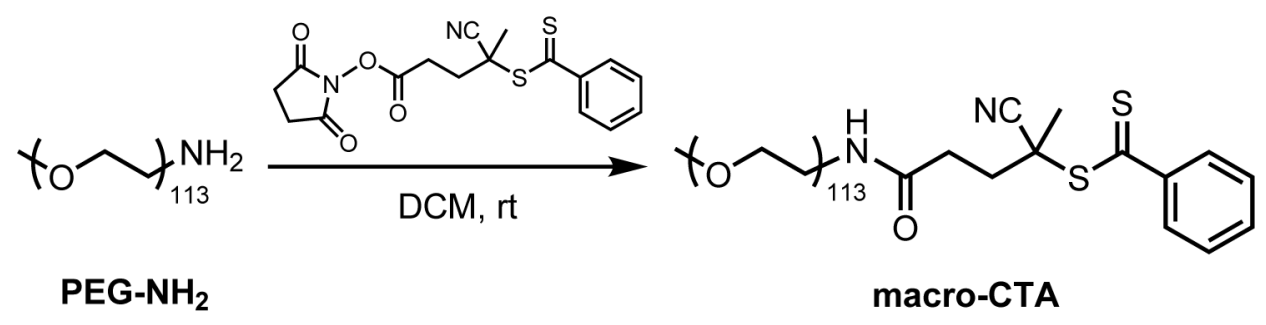


macro-CTA (macromolecular chain transfer agent), a linear PEG-dithiobenzoate derivative. The compound was synthesized based on a previous report with modifications. ${ }^{2}$ PEG- $\mathrm{NH}_{2}$ (JenKem, Cat\# A3035-1/M-NH2HCl-5000, $1.0 \mathrm{~g}, 0.20 \mathrm{mmol}, 1.0$ equiv., $M_{\mathrm{n}}$ 5000) was dissolved in $2.4 \mathrm{~mL}$ dichloromethane (DCM). A solution of 4-cyano-4-(phenylcarbonothioylthio)pentanoic acid N-succinimidyl ester (150 mg, $0.4 \mathrm{mmol}, 2.0$ equiv.) in $0.6 \mathrm{~mL}$ DCM was added dropwise into the mixture at room temperature and stirred for overnight. Next, the solution was precipitated into excess diethyl ether. The pink solid was re-dissolved in DCM and dialyzed against DCM using 1 kD MWCO dialysis tubing for 24 hours. The product, macro-CTA was dried under vacuum and obtained as pink powder $(712 \mathrm{mg}) .{ }^{1} \mathrm{H}$ NMR (500 MHz, $\left.\mathrm{CDCl}_{3}\right) \delta 7.92-7.89(\mathrm{~m}), 7.58-7.54(\mathrm{~m}), 7.42-7.37(\mathrm{~m}), 3.77(\mathrm{~d}), 3.64(\mathrm{~s}, 448 \mathrm{H}), 3.51$ (s), $3.51-3.42(\mathrm{~m}), 3.38$ (s), $2.67-2.51(\mathrm{~m}), 2.42$ (ddd), 1.94 (s) (Figure SP13). ${ }^{13} \mathrm{C}$ NMR $(125 \mathrm{MHz}$ $\left.\mathrm{CDCl}_{3}\right)$ (Figure SP14). GPC (THF), $M_{\mathrm{n}}: 7.6 \mathrm{~K}, \emptyset: 1.03$ (Figure SP15).

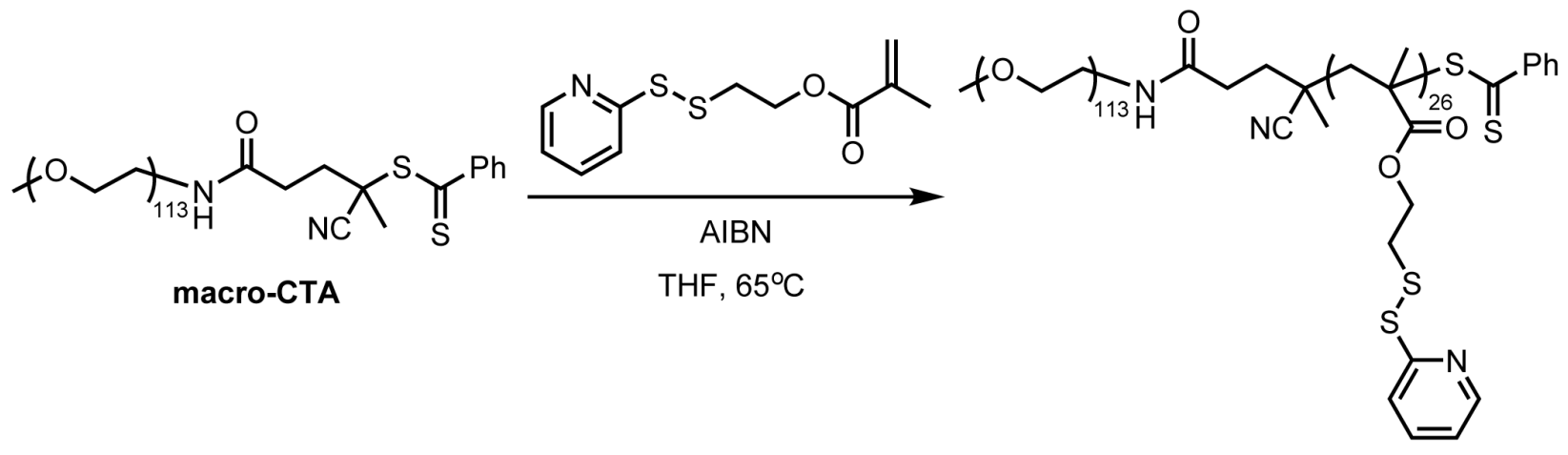

B73

B73, an amphiphilic block copolymer. Pyridyl disulfide ethyl methacrylate $(237 \mathrm{mg}, 0.93 \mathrm{mmol}, 31.0$ equiv.), macro-CTA (150 mg, 0.03 mmol, 1.0 equiv., $\left.M_{\mathrm{n}} \sim 5000\right)$, and AIBN (1.0 mg, $0.006 \mathrm{mmol}, 0.2$ equiv.) were dissolved in $0.8 \mathrm{~mL}$ anhydrous tetrahydrofuran (THF) and transferred to a 10-mL Schlenk flask. The flask was sealed and the reaction mixture was degassed with four freeze-pump-thaw cycles. After refilling the flask with an argon atmosphere, the reaction was dipped into a prewarmed oil bath at $65^{\circ} \mathrm{C}$ and stirred for 18 hours. Next, the polymerization was quenched by freezing the mixture with liquid 
nitrogen and exposing to air. The polymer was purified by dissolving in $\mathrm{CHCl}_{3}$ and subsequently precipitating in cold diethyl ether for three times. The product was dried under vacuum and obtained as

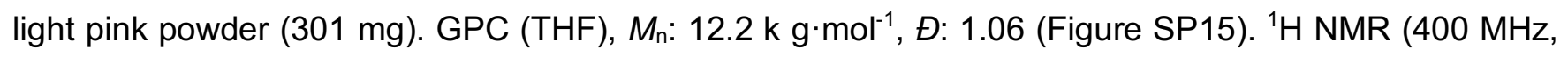
$\left.\mathrm{CDCl}_{3}\right) \delta 8.44$ (s), 7.64 (s), 7.08 (s), 4.21 (s), 3.64 (s), 3.38 (s), 3.01 (s), 1.93 (s), 1.85 (s), 1.06 (s), 0.92 (s) (Figure SP16). The molar ratio between two repeating units was determined by integrating the aromatic proton in the pyridine and the methoxy proton $(\delta 3.3)$ in the polyethylene glycol side chain. The degree of polymerization for PDS unit was $\left.\sim 26 .{ }^{13} \mathrm{C} \mathrm{NMR} \mathrm{(100} \mathrm{MHz,} \mathrm{CDCl}\right)$ (Figure SP17). IR spectrum and TGA curve (Figure SP18).

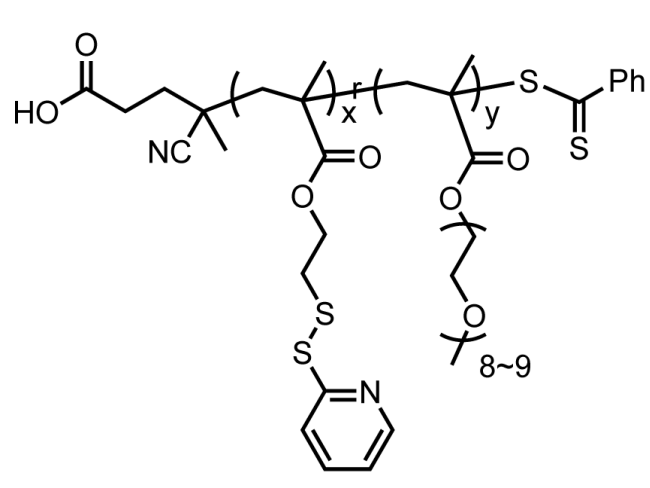

R73

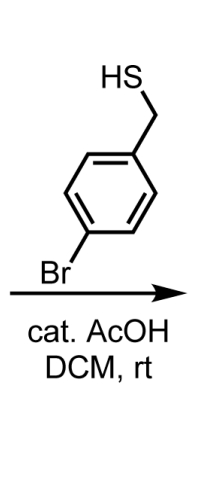<smiles>[TlH]</smiles>

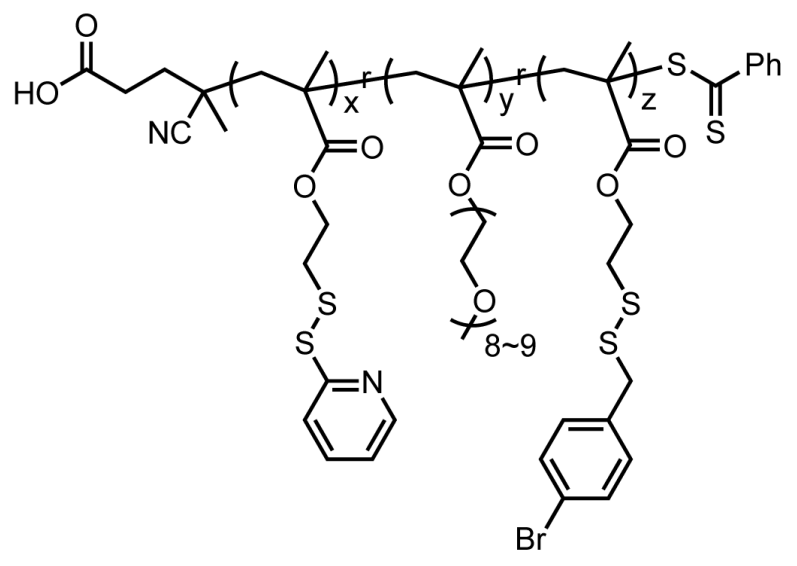

R73-Br

$\mathbf{R 7 3 - B r}$, an amphiphilic random copolymer. $\mathbf{R 7 3}(100 \mathrm{mg}$ ) was dissolved in $2 \mathrm{~mL} \mathrm{CHCl} 3$ and stirred at room temperature. 4-Bromobenzyl mercaptan $(6.17 \mathrm{mg}, 0.03 \mathrm{mmol}, 0.14$ equiv. vs. the pyridyl disulfide moieties within R73) and acetic acid ( $3 \mu \mathrm{L})$ were added into the reaction mixture and stirred for 6 hours. The polymer was purified by dialyzing against $\mathrm{CH}_{2} \mathrm{Cl}_{2}$ using $1 \mathrm{kD}$ MWCO dialysis tubing for 24 hours. The product was dried under vacuum and obtained as pink oil (125 mg, quantitative). GPC (THF), $M_{\mathrm{n}}$ : $8.1 \mathrm{k} \mathrm{g} \cdot \mathrm{mol}^{-1}$, $, \mathrm{:}: 1.21 .{ }^{1} \mathrm{H} \mathrm{NMR}\left(500 \mathrm{MHz}, \mathrm{CDCl}_{3}\right) \delta 8.46$ (s), 7.67 (s), 7.44 (s), 7.20 (s), 7.10 (s), 4.22 (s), $4.08(\mathrm{~s}), 3.83(\mathrm{~s}), 3.70-3.56(\mathrm{~m}), 3.54(\mathrm{~s}), 3.37(\mathrm{~s}), 3.02(\mathrm{~s}), 2.59(\mathrm{~s}), 1.92(\mathrm{~s}), 1.83(\mathrm{~s}), 1.05(\mathrm{~s}), 0.88(\mathrm{~s})$ (Figure SP19). The molar ratio between three repeating units was determined by integrating the aromatic 
proton in the pyridine, the methoxy proton $(\delta 3.3)$ in the polyethylene glycol side chain, and the methylene proton next to the phenyl group (x:y:z $\left.=0.60: 0.25: 0.15) .{ }^{13} \mathrm{C} \mathrm{NMR} \mathrm{(100} \mathrm{MHz,} \mathrm{CDCl}_{3}\right)($ Figure SP20). IR spectrum and TGA curve (Figure SP21).

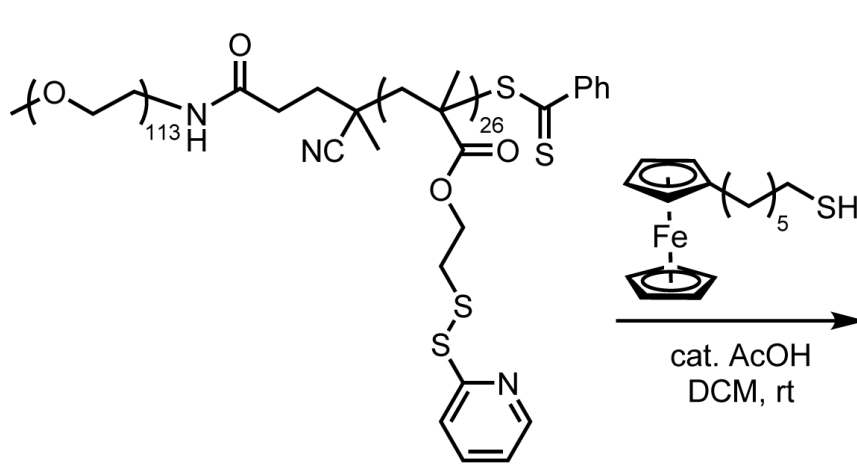

B73

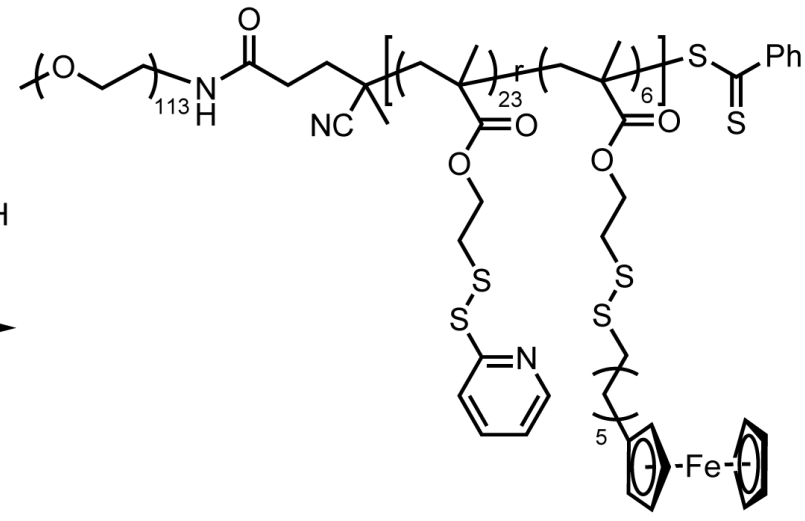

B73-Fe

B73-Fe, an amphiphilic block copolymer consisting of a linear PEG block and a block with PDS and ferrocene derivatives randomly distributed. B73 $(50 \mathrm{mg})$ was dissolved in $2 \mathrm{~mL} \mathrm{CHCl} 3$ and stirred at room temperature. 6-(Ferrocenyl)hexanethiol $(5.28 \mathrm{mg}, 0.016 \mathrm{mmol}, 0.14$ equiv. vs. the pyridyl disulfide moieties within $\mathbf{R 7 3}$ ) and acetic acid $(1.5 \mu \mathrm{L})$ were added into the reaction mixture and stirred for 6 hours. The polymer was purified by dialyzing against $\mathrm{CH}_{2} \mathrm{Cl}_{2}$ using $1 \mathrm{kD}$ MWCO dialysis tubing for 24 hours. The product was dried under vacuum and obtained as yellow oil (71 mg, quantitative). GPC (THF), $M_{\mathrm{n}}$ : $13.4 \mathrm{k} \mathrm{g} \cdot \mathrm{mol}^{-1}, \oplus: 1.15 .{ }^{1} \mathrm{H}$ NMR $\left(500 \mathrm{MHz} \mathrm{CDCl}_{3}\right)$ ठ 8.45 (s), 7.65 (s), 7.08 (s), 4.22 (s), 4.08 (s), 4.03 (s), 3.64 (s), 3.38 (s), 3.02 (s), 2.85 (s), 2.67 (s), 2.31 (s), 1.93 (s), 1.84 (s), 1.06 (s), 0.90 (s) (Figure SP22). The molar ratio between three repeating units was determined by integrating the aromatic proton in the pyridine, the methoxy proton $(\delta 3.3)$ in the polyethylene glycol chain end, and the methylene proton next to the disulfide bond within the ferrocenyl-terminated side chain. ${ }^{13} \mathrm{C} \mathrm{NMR}\left(125 \mathrm{MHz}, \mathrm{CDCl}_{3}\right)(\mathrm{Figure}$ SP23). IR spectrum and TGA curve (Figure SP24). 


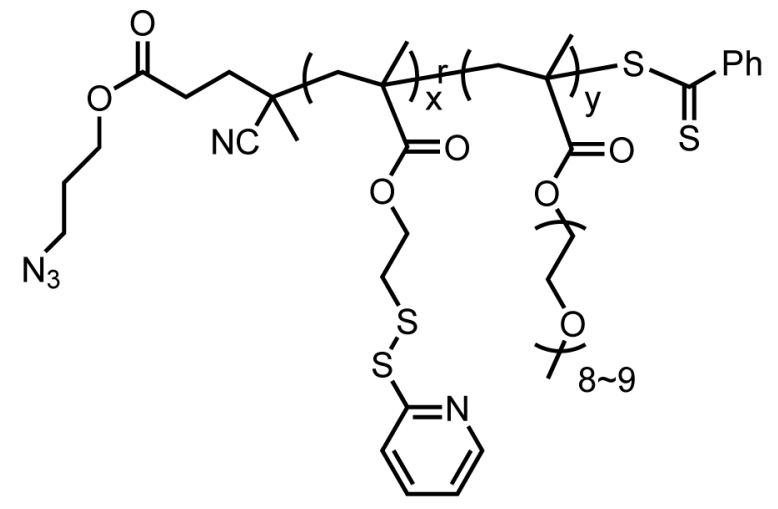

R73- $\mathrm{N}_{3}$

R73- $\mathbf{N}_{3}$, an amphiphilic random copolymer with an azide group installed on the $\alpha$-end. Next, R73-N $\mathbf{N}_{3}$ was labeled with DBCO-Cy3, resulting in a Cy3-labeled amphiphilic random copolymer (denoted as R73-Cy3). The polymer synthesis and labeling was conducted based on our previous report. ${ }^{3}$<smiles>CC(C)(C)CCC(=O)NCCCC(C)(C)CC(C)(C)C(=O)OCCSSc1ccccn1</smiles>

B73
Az-ACVA

THF, $65^{\circ} \mathrm{C}$<smiles>CC(C)OCCNC(=O)CCC(C)(C)CC(C)(C)C(CC(C)(C)CCC(=O)OCCCN)(C(=O)O)C(C)(C)C</smiles><smiles>CC(C)(N)N=NC(C)(C)CCC(=O)OCCCN</smiles>

Az-ACVA

B73- $\mathbf{N}_{3}$, an amphiphilic block copolymer consisting of a linear PEG block and a PDS block, with an azide group modified on the $\omega$-end. Compound Az-ACVA was synthesized based on our previous report. ${ }^{3}$ B73 (120 mg) and Az-ACVA (93 mg, $0.21 \mathrm{mmol}, 20$ equiv. vs. the dithioester end group within B73) were dissolved in $760 \mu \mathrm{L}$ anhydrous tetrahydrofuran (THF) and transferred to a 10-mL Schlenk flask. The flask 
was sealed and the reaction mixture was degassed with four freeze-pump-thaw cycles. After refilling the flask with an argon atmosphere, the reaction was dipped into a prewarmed oil bath at $65{ }^{\circ} \mathrm{C}$ and stirred for 4 hours. The polymer was purified by dialyzing against acetone using 1 kD MWCO dialysis tubing for 24 hours. The product was dried under vacuum and obtained as white powder (88 mg). ${ }^{1} \mathrm{H}$ NMR (500 $\left.\mathrm{MHz}, \mathrm{CDCl}_{3}\right) \delta 8.44$ (s), 7.66 (s), 7.08 (s), 4.21 (s), 4.08 (s), 3.64 (s), 3.38 (s), 3.01 (s), 1.93 (s), 1.85 (s), 1.06 (s), 0.90 (s) (Figure SP25). The molar ratio between two repeating units was determined by integrating the aromatic proton in the pyridine and the methoxy proton ( $\delta 3.3)$ in the polyethylene glycol side chain. The conversion of the $\omega$-end group was validated by the color change of the polymer (Figure S36) and the appearance of azide group in infrared spectroscopy. ${ }^{13} \mathrm{C} \mathrm{NMR}\left(125 \mathrm{MHz}, \mathrm{CDCl}_{3}\right)($ Figure SP26). IR spectrum (Figure SP27). GPC (THF), $M_{\mathrm{n}}: 10.9 \mathrm{k} \mathrm{g} \cdot \mathrm{mol}^{-1}, Ð: 1.07$ (Figure SP28). In the GPC curve, the shoulder peak with higher molecular weight distribution indicates ineffective radical trapping and the formation of termination product (dimer). ${ }^{4}$

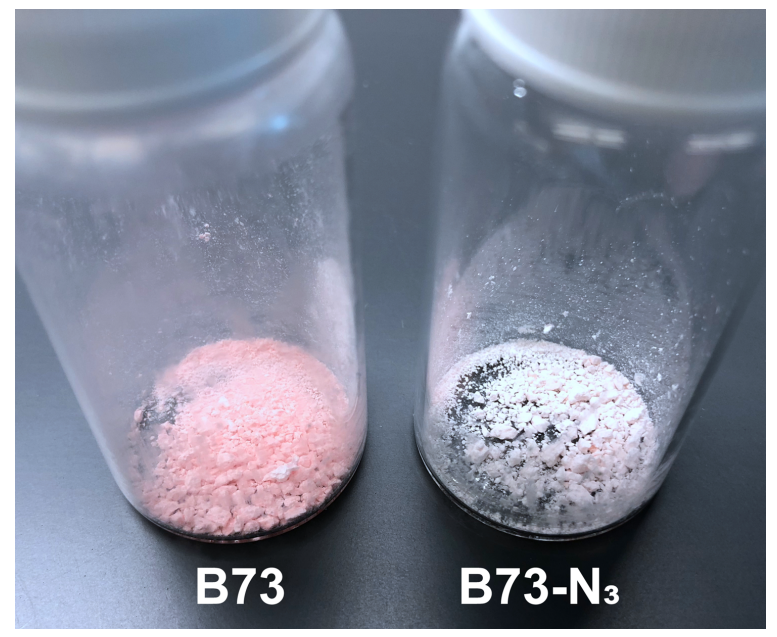

Figure S36. Photograph of the color change from B73 to B73- $\mathbf{N}_{\mathbf{3}}$. 

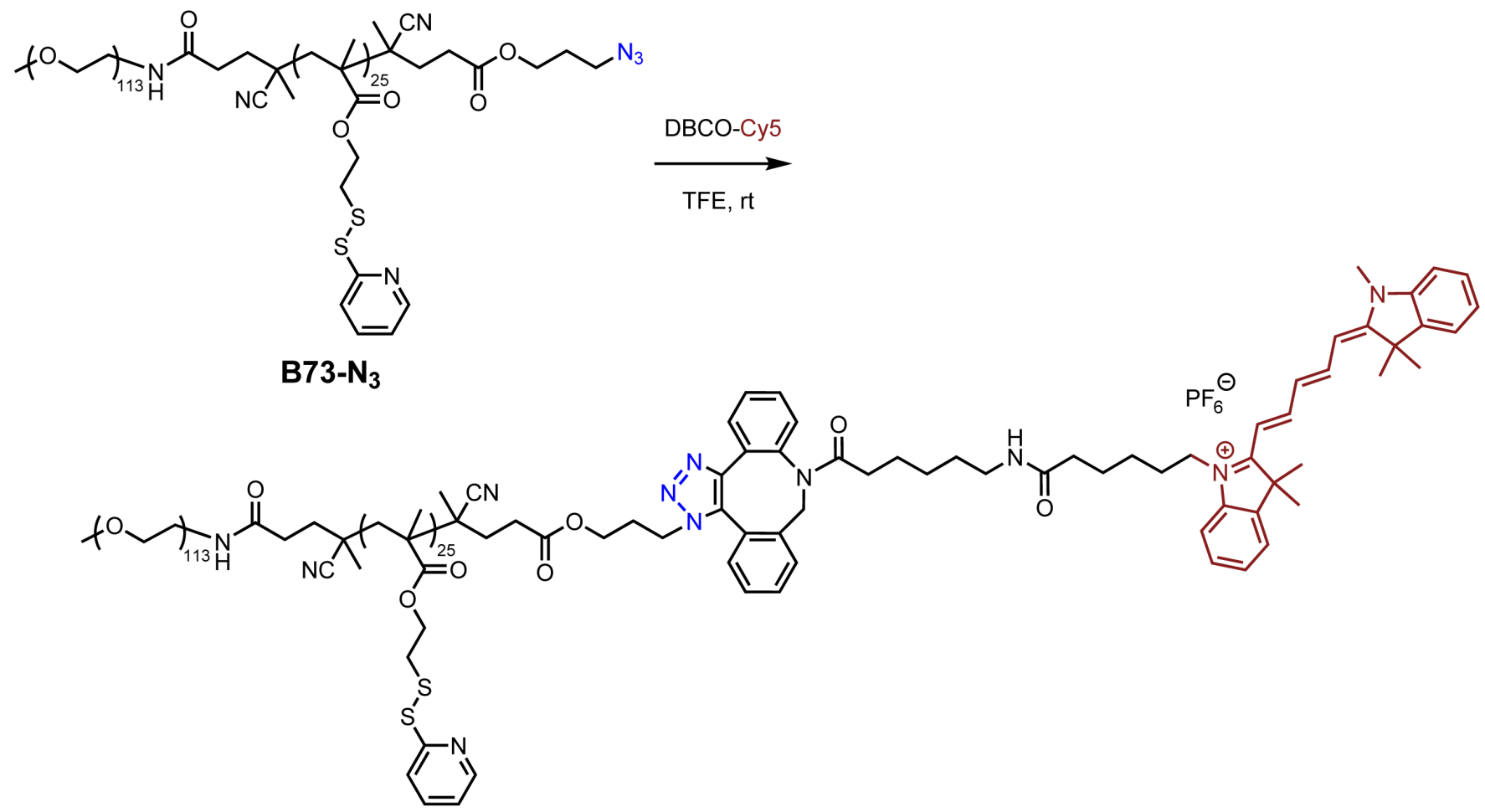

\section{B73-Cy5}

B73-Cy5, an amphiphilic block labeled with Cy5 on the $\omega$-end. B73-N $\mathbf{3}$ and DBCO-Cy5 (Lumiprobe, Cat\# C30F0, 1.05 equiv. vs. the azide group on B73- $\left.\mathbf{N}_{3}\right)$ were dissolved in trifluoroethanol $(2 \mathrm{~mL})$ and stirred at room temperature for 24 hours. The purification process was adapted from our previous report. ${ }^{3}$ The solvent was evaporated and the mixture was purified by gel permeation chromatography over SorbaDex 20-LH gel filtration matrix (Cat\# 801009). A mixture of $\mathrm{CHCl}_{3} / \mathrm{MeOH}(v / v=3: 1)$ was used as the eluent. The SorbaDex 20-LH matrix was dispersed in the eluent for at least 1 hour before packing the column. The high-molecular-weight fraction was collected and dried under vacuum, quantitatively resulting in B73Cy5.

\subsection{Scanning electron microscopy (SEM)}

The sample for SEM imaging was prepared by allowing a droplet of the aqueous solution containing R73B73 co-assemblies $\left(0.1 \mathrm{mg} \cdot \mathrm{mL}^{-1}\right)$ to dry on a low resistivity silicon wafer (0 100 Ohm spec). The samples 
were used as prepared without sputter coating. Imaging was performed on a FEI/ThermoFisher Magellan $400 \mathrm{HRSEM}$ at $2 \mathrm{kV}$ landing voltage (100 V deceleration to help with charging) and $3.1 \mathrm{pA}$ beam current.

\subsection{FRET evaluation of polymeric co-assemblies between $R 73$ and $B 73$}

A mixture of R73-Cy3 and B73-Cy5 ( $w / w=1: 1,0.1 \mathrm{mg}$ in total) were dissolved in $100 \mu \mathrm{L}$ acetone.

Deionized $\mathrm{H}_{2} \mathrm{O}(1 \mathrm{~mL})$ was added dropwise into the acetone solution while stirring. Next, a calculated amount of dithiothreitol (DTT) stock solution was added into the mixture in one portion to aim at $20 \mathrm{~mol} \%$ crosslinking of the PDS units. The solution was stirred overnight to evaporate the organic solvent. The polymeric assemblies were purified by centrifugal filtration using an Amicon Ultra-0.5 centrifugal filter ( 3 k MWCO). The final volume of the solution was adjusted to $1 \mathrm{~mL}$ with deionized $\mathrm{H}_{2} \mathrm{O}$. The R73-Cy3-B73$\mathbf{N}_{3}$ and R73-N $\mathbf{N}_{3}-\mathbf{B} 73-$ Cy5 $(w / w=1: 1)$ co-assemblies were also prepared following the above procedures as control groups. The FRET experiment was performed using a PerkinElmer LS 55 fluorescence spectrometer with an excitation wavelength at $540 \mathrm{~nm}$.

\subsection{Molecular weight measurement of polymeric (co-)assemblies using static light scattering}

Different mass ratios of copolymers (2 mg, individual or blended) were dissolved in $200 \mu \mathrm{L}$ acetone. Deionized $\mathrm{H}_{2} \mathrm{O}(2 \mathrm{~mL})$ was added dropwise into the acetone solution while stirring. A calculated amount of dithiothreitol (DTT) $\left(1 \mathrm{mg} \cdot \mathrm{mL}^{-1}\right.$, freshly prepared in $\left.\mathrm{H}_{2} \mathrm{O}\right)$ was added into the mixture in one portion to aim at 20 mol\% crosslinking of the PDS units. The solution was stirred overnight to allow the evaporation of the organic solvent. Next, the polymeric assemblies were purified by centrifugal filtration using an Amicon Ultra-0.5 centrifugal filter ( $3 \mathrm{k} \mathrm{MWCO}$ ) and the final volume of the solution was adjusted to $2 \mathrm{~mL}$ with deionized $\mathrm{H}_{2} \mathrm{O}$. The static light scattering (SLS) experiments were performed using a Malvern NanoZS instrument with toluene as standard and water as the solvent. Toluene and water were pre-filtered by passing through a syringe filter unit with $0.22 \mu \mathrm{m}$ pore size. The refractive index increment $(\mathrm{dn} / \mathrm{dc})$ of the co-assemblies were approximated to be 0.133 (the refractive index increment of polyethylene glycol, a major component the copolymers in the current study). The samples were pre-equilibrated for $60 \mathrm{~s}$ before 
measurement. Debye plot was employed to analyze the SLS data using at least five different concentrations per group.

To calculate the aggregation number $(N)$ in each (co-)assembly, the weight average molecular weight of polymeric assemblies obtained from SLS analysis was first calibrated to compensate the molecular weight loss during DTT-induced crosslinking (i.e. adding the cleaved 2-pyridinethione portion). The weight average molecular weight $\left(M_{\mathrm{w}}\right)$ of copolymers was based on THF GPC measurement: (a) For blended copolymers, the value was calculated based on the molar ratio and $M_{w}$ (measured by THF GPC) of each copolymer component; (b) For individual copolymers, the value was the $M_{w}$ from THF GPC measurement. Finally, the aggregation number was calculated using (Calibrated molecular weight of crosslinked assemblies based on SLS results / Weight average molecular weight of copolymers based on THF GPC results).

\subsection{Evaluation of critical aggregation concentration of polymeric (co-)assemblies}

Copolymers (individual or blended) were dissolved in $100 \mu \mathrm{L}$ acetone. Deionized $\mathrm{H}_{2} \mathrm{O}(1 \mathrm{~mL})$ was added dropwise into the acetone solution while stirring. The stock solution $\left(1 \mathrm{mg} \cdot \mathrm{mL}^{-1}\right)$ of Nile red was freshly prepared before loading into polymeric assemblies. A calculated amount of Nile red solution for 10 wt $\%$ loading was added into the $\mathrm{H}_{2} \mathrm{O} /$ acetone $(v / v=10: 1)$ solution that contains polymers. Next, a calculated amount of dithiothreitol (DTT) stock solution was added into the mixture in one portion to aim at $20 \mathrm{~mol} \%$ crosslinking of the PDS units. The solution was stirred overnight to evaporate the organic solvent. Unencapsulated free dyes were removed by passing the solution through a cotton-plugged glass Pasteur pipette (Figure S35). The volume of the solution was then adjusted with $\mathrm{H}_{2} \mathrm{O}$ to result in the desired concentration of polymeric assemblies. The Nile red loading was represented by measuring the fluorescence intensity at $628 \mathrm{~nm}$ with an excitation wavelength at $552 \mathrm{~nm}$. For the CAC analysis, the Nile red loading of polymeric assemblies was measured at serially diluted concentrations. In each (co-)assembly group, the Nile red fluorescence intensity (at $628 \mathrm{~nm}$ ) at the highest tested polymer concentration was normalized as $100 \%$ for the CAC analysis. 


\subsection{Cell culture}

All cells used in the study were cultured in a humidified atmosphere $\left(5 \% \mathrm{CO}_{2}\right)$ at $37^{\circ} \mathrm{C}$. HeLa cell line was purchased from ATCC (Cat\# CCL-2). EMT6 cell line was a gift from Dr. Michelle E. Farkas (UMass Amherst). Cells were grown and passaged in Dulbecco's modified eagle medium: Nutrient mixture F-12 + 1× GlutaMAX (DMEM, ThermoFisher, Cat\# 10565018) supplemented with $10 \%$ fetal bovine serum (FBS) and $1 \%$ antibiotics $\left(100 \mathrm{U} \cdot \mathrm{mL}^{-1}\right.$ penicillin-streptomycin).

\subsection{Detection of cellular ATP level and evaluation of cellular metabolic activity}

A total of $10 \mathrm{k}$ HeLa or EMT6 cells were cultured in a 96 -well plate for 24 hours prior to the experiment. A stock solution of the R73-B73 ( $w / w=1: 1)$ co-assemblies with $20 \%$ crosslinking was made before the experiment. The stock solution was serially 2-fold diluted into full DMEM growth medium. Next, the complete cell growth medium was replaced by the polymer-containing medium and incubated for 24 hours. Before each measurement, cells were washed with phosphate buffer saline (PBS). For the detection of cellular ATP level, CellTiter-Glo luminescent assay (Promega, Ca\#\# G7572) was used. The washed cells were incubated with $100 \mu \mathrm{L}$ CellTiter-Glo reagent at room temperature with orbital shaking for 2 minutes, followed by another 10-minute incubation without shaking. Next, the luminescence of each sample was measured to evaluate the ATP level. To measure the cellular metabolic activity, AlamarBlue assay (ThermoFisher, Cat\# DAL1100) was employed. The washed cells were incubated with $220 \mu \mathrm{L}$ full DMEM growth medium containing $10 \%$ alamarBlue reagent for 60 minutes. Cellular metabolic activity was calculated by measuring the fluorescence intensity of AlamarBlue at $590 \mathrm{~nm}$, with an excitation wavelength at $535 \mathrm{~nm}$. In each assay, after subtracting background intensity from the reagent only group (no cells), the readout from the cells in the control group (growing in DMEM full growth medium for 24hour) was normalized as $100 \%$. 


\section{Spectral data}

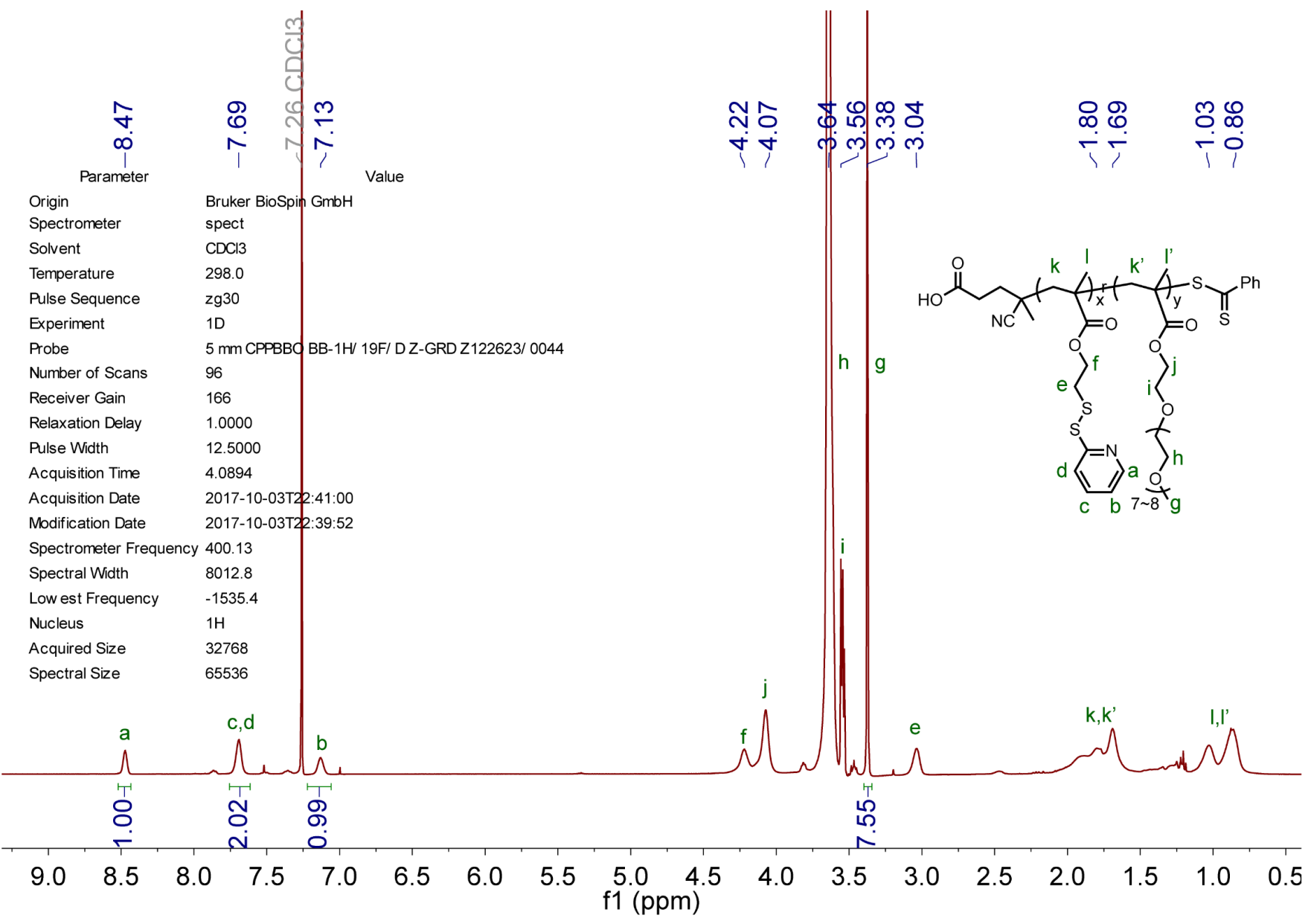

Figure SP1. ${ }^{1} \mathrm{H}$ NMR spectrum $(400 \mathrm{MHz})$ of $\mathbf{R} 37$ in $\mathrm{CDCl}_{3}$. 


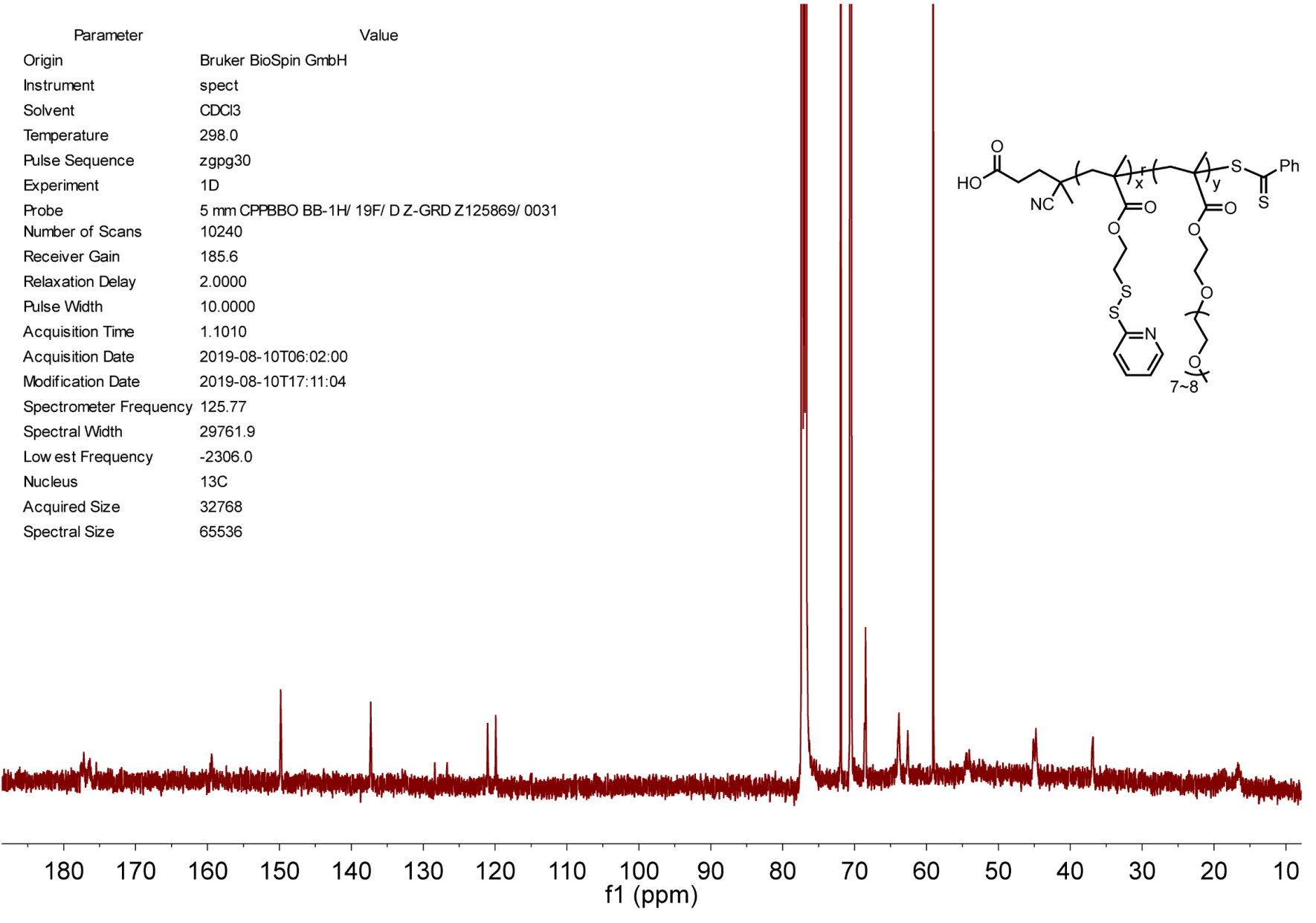

Figure SP2. ${ }^{13} \mathrm{C}$ NMR spectrum $(125 \mathrm{MHz})$ of $\mathbf{R} 37$ in $\mathrm{CDCl}_{3}$. 
a)
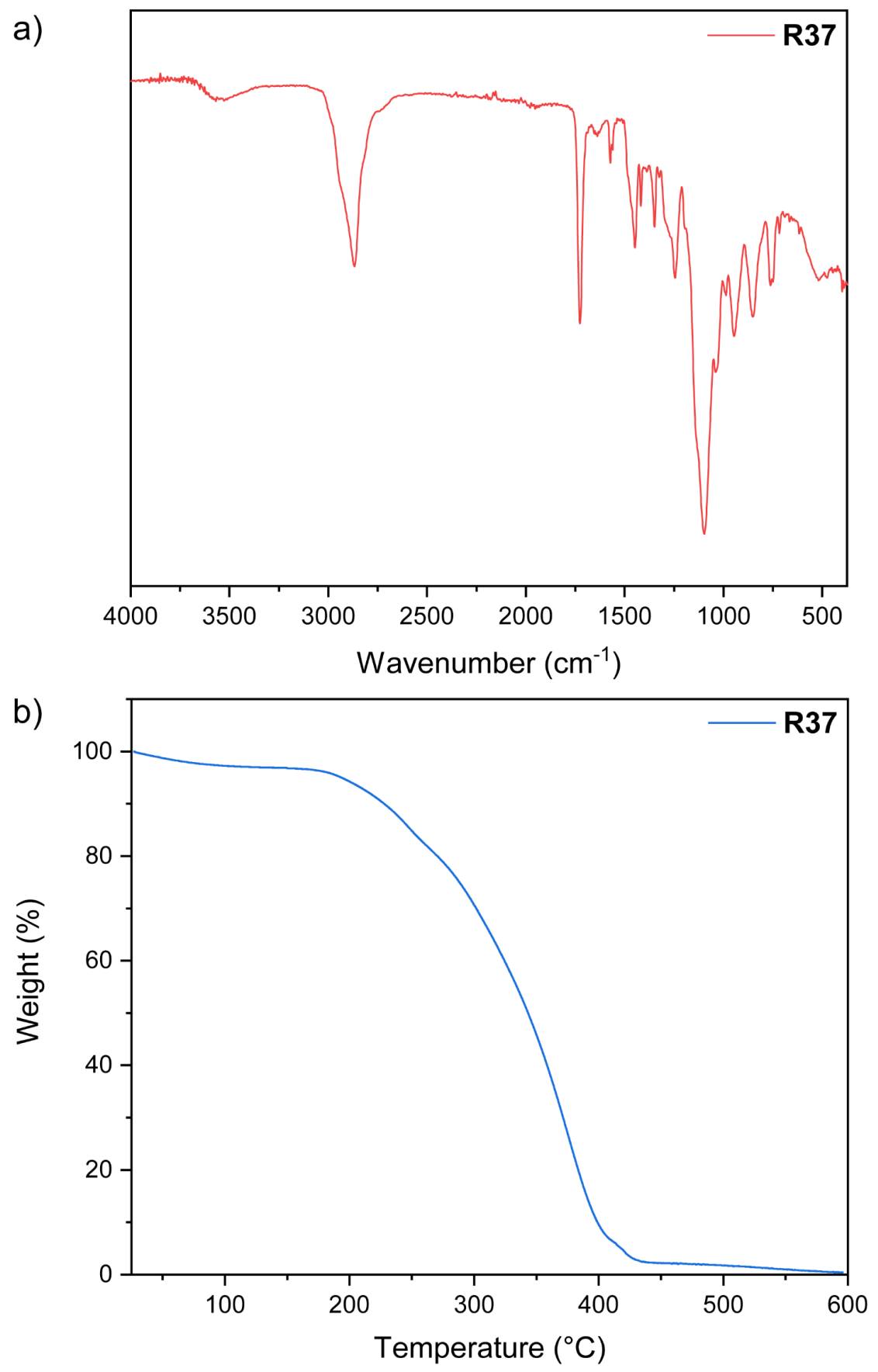

Figure SP3. (a) IR spectrum and (b) TGA curve of R37. 


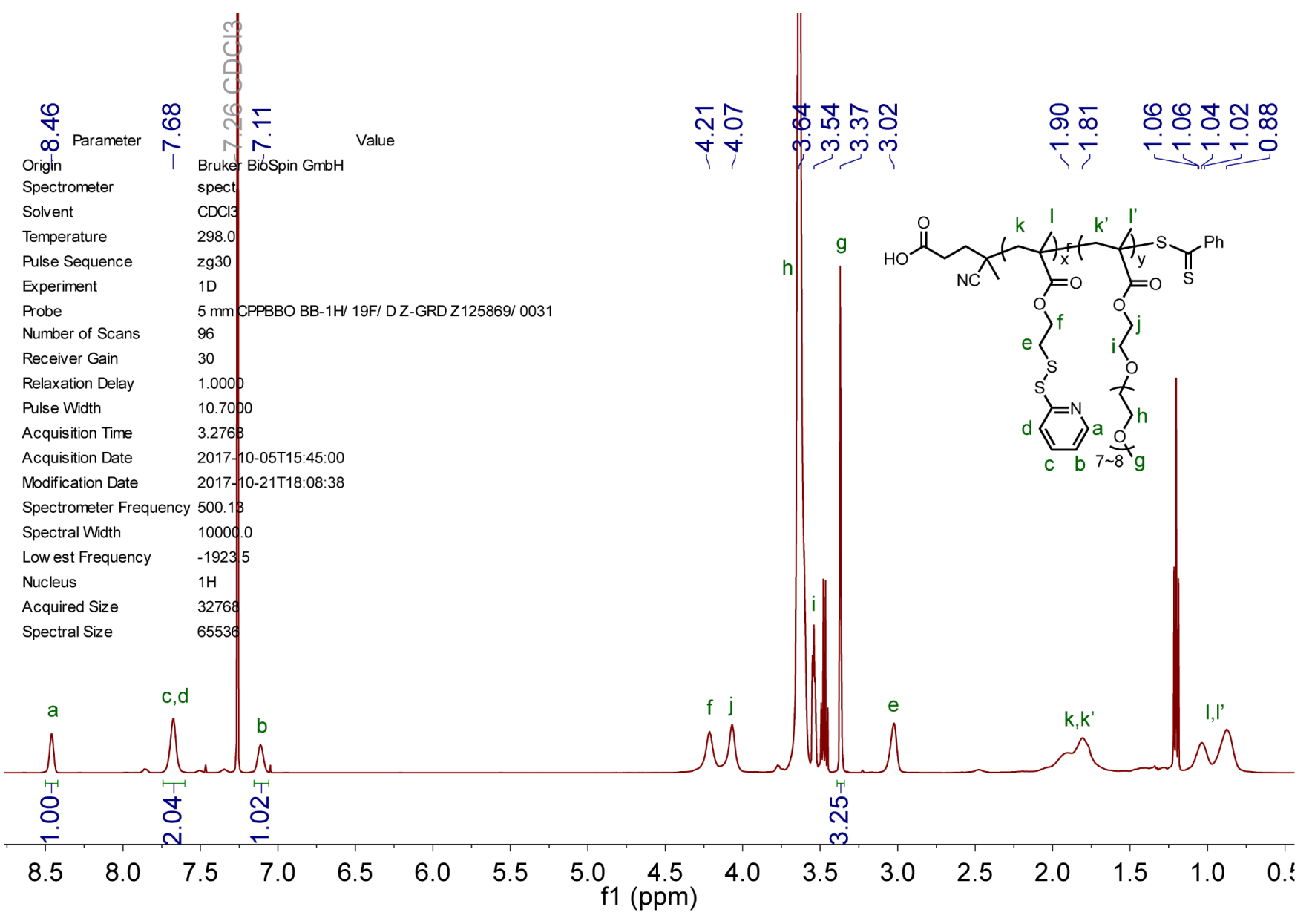

Figure SP4. ${ }^{1} \mathrm{H}$ NMR spectrum $(500 \mathrm{MHz})$ of $\mathbf{R} 55$ in $\mathrm{CDCl}_{3}$. 

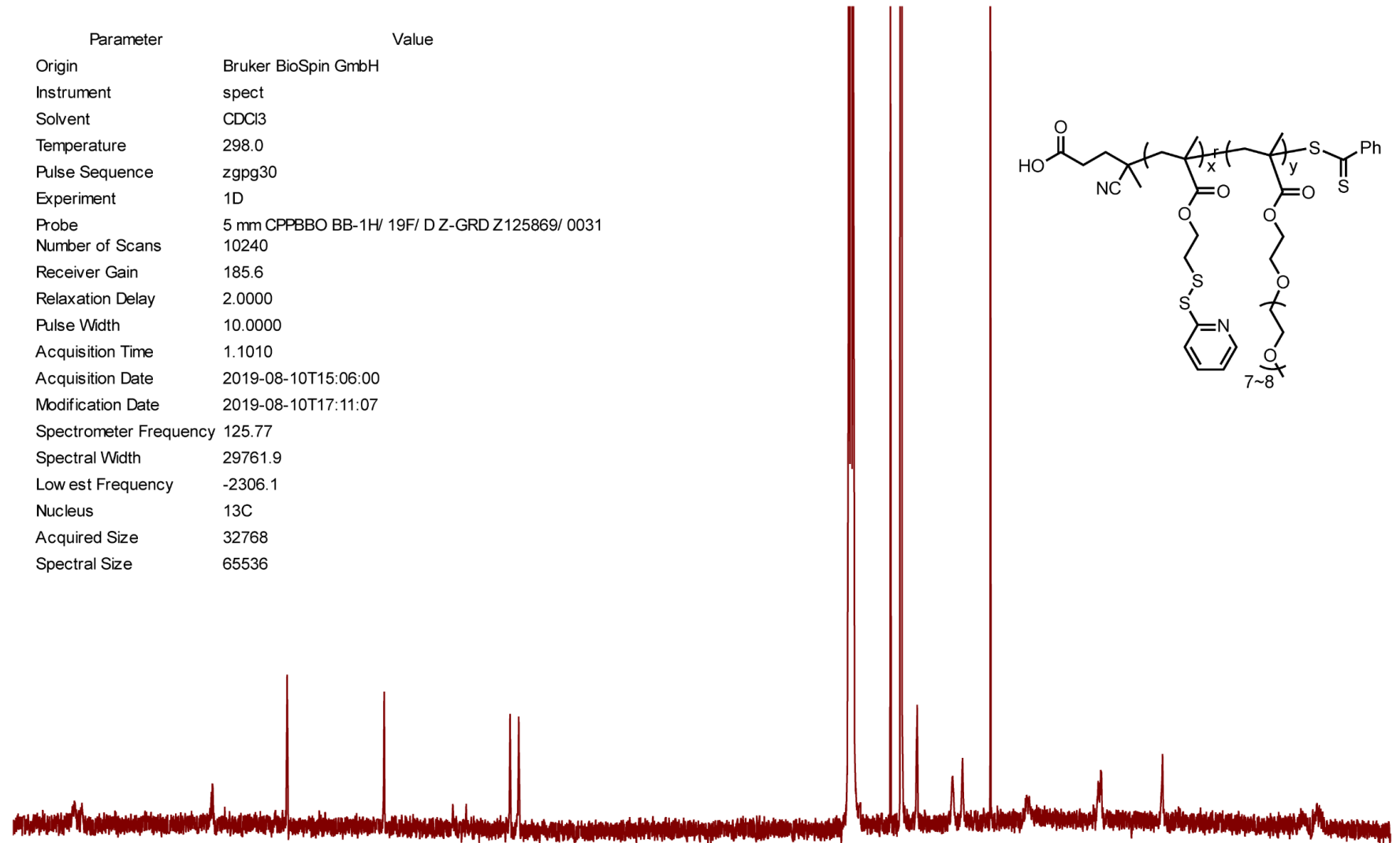

$\begin{array}{llllllllllllllllll}180 & 170 & 160 & 150 & 140 & 130 & 120 & 110 & \begin{array}{l}100 \\ \mathrm{f} 1(\mathrm{ppm})\end{array} & 80 & 70 & 60 & 50 & 40 & 30 & 20 & 10\end{array}$

Figure SP5. ${ }^{13} \mathrm{C}$ NMR spectrum $(125 \mathrm{MHz})$ of $\mathbf{R} 55$ in $\mathrm{CDCl}_{3}$. 
a)

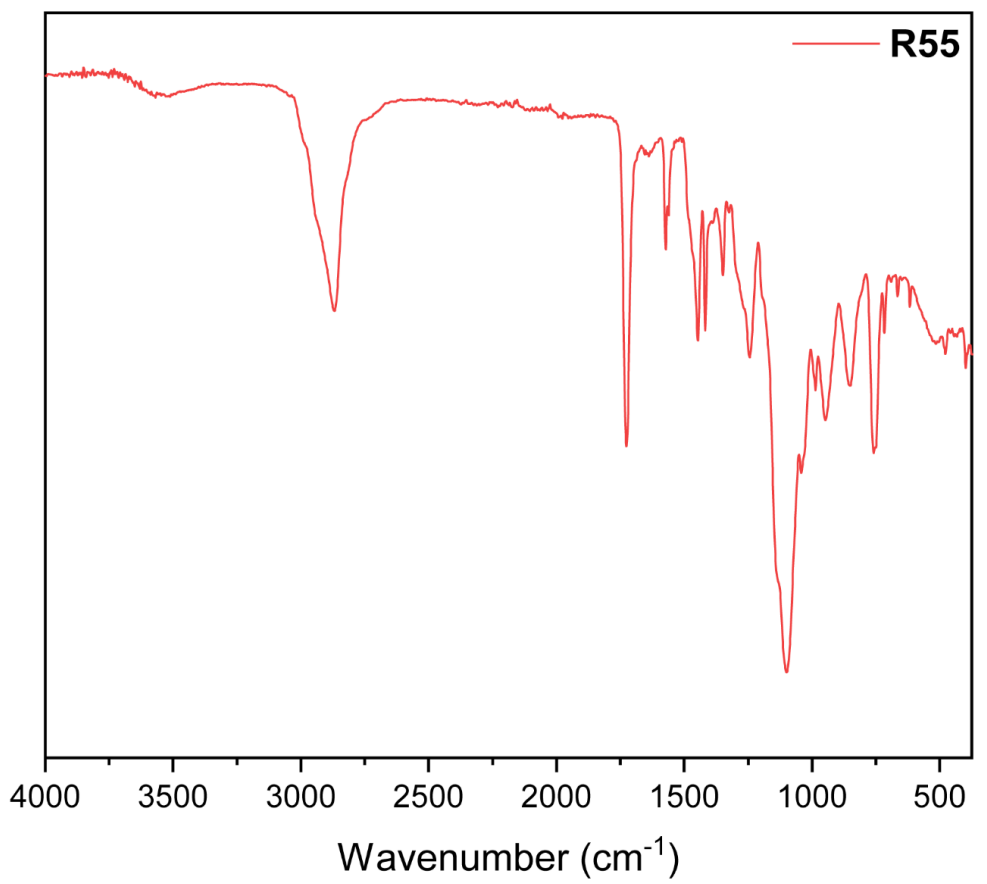

b)

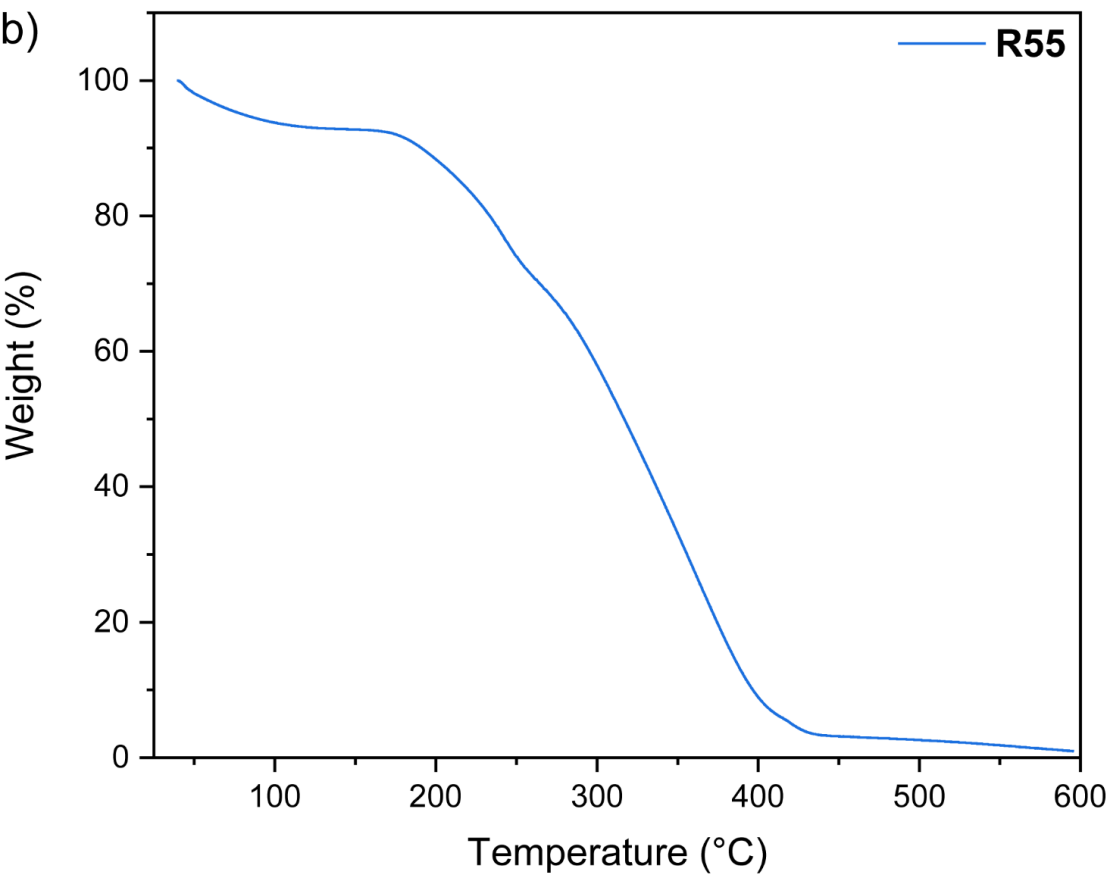

Figure SP6. (a) IR spectrum and (b) TGA curve of R55. 


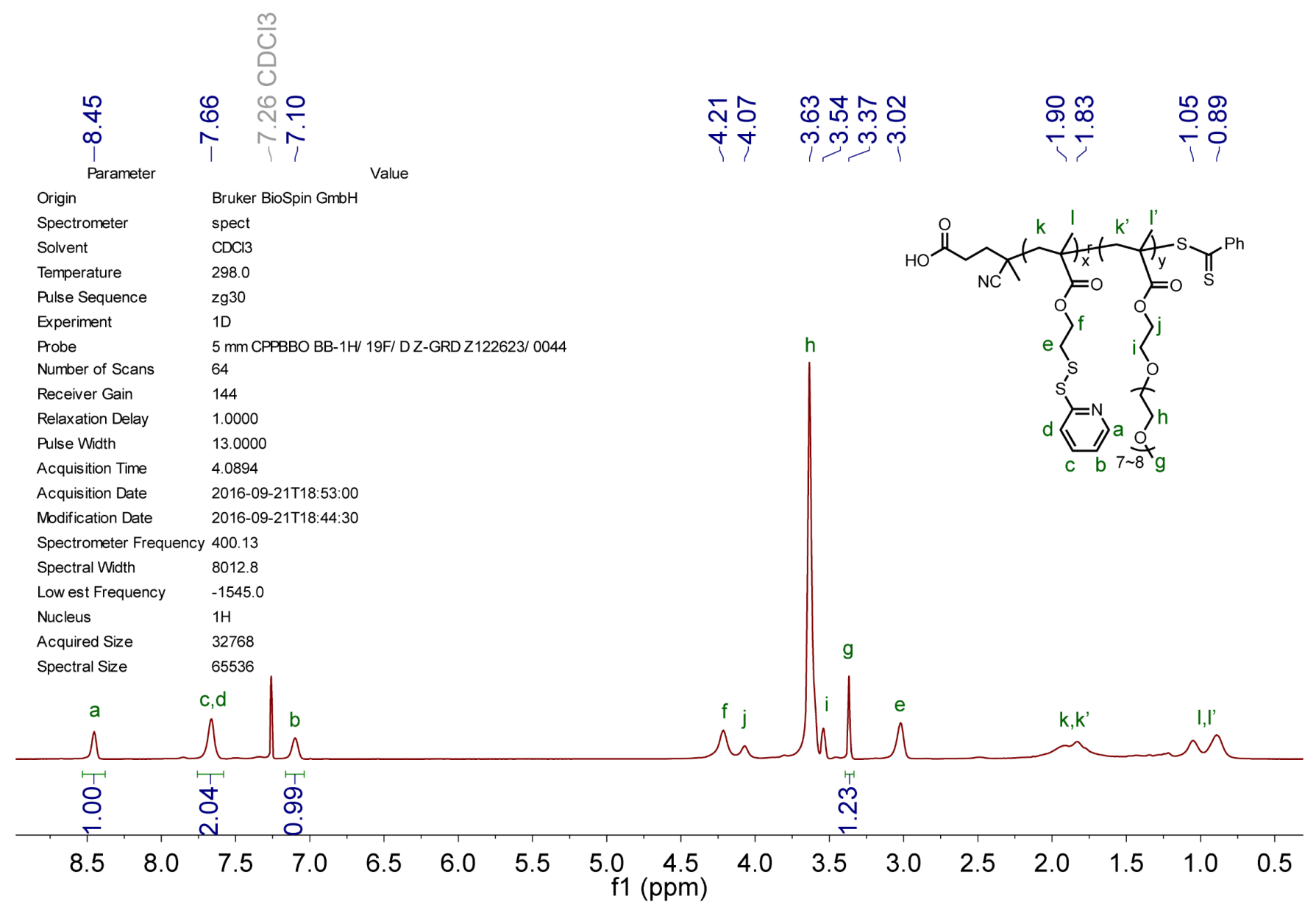

Figure SP7. ${ }^{1} \mathrm{H}$ NMR spectrum $(400 \mathrm{MHz})$ of $\mathbf{R} 73$ in $\mathrm{CDCl}_{3}$. 


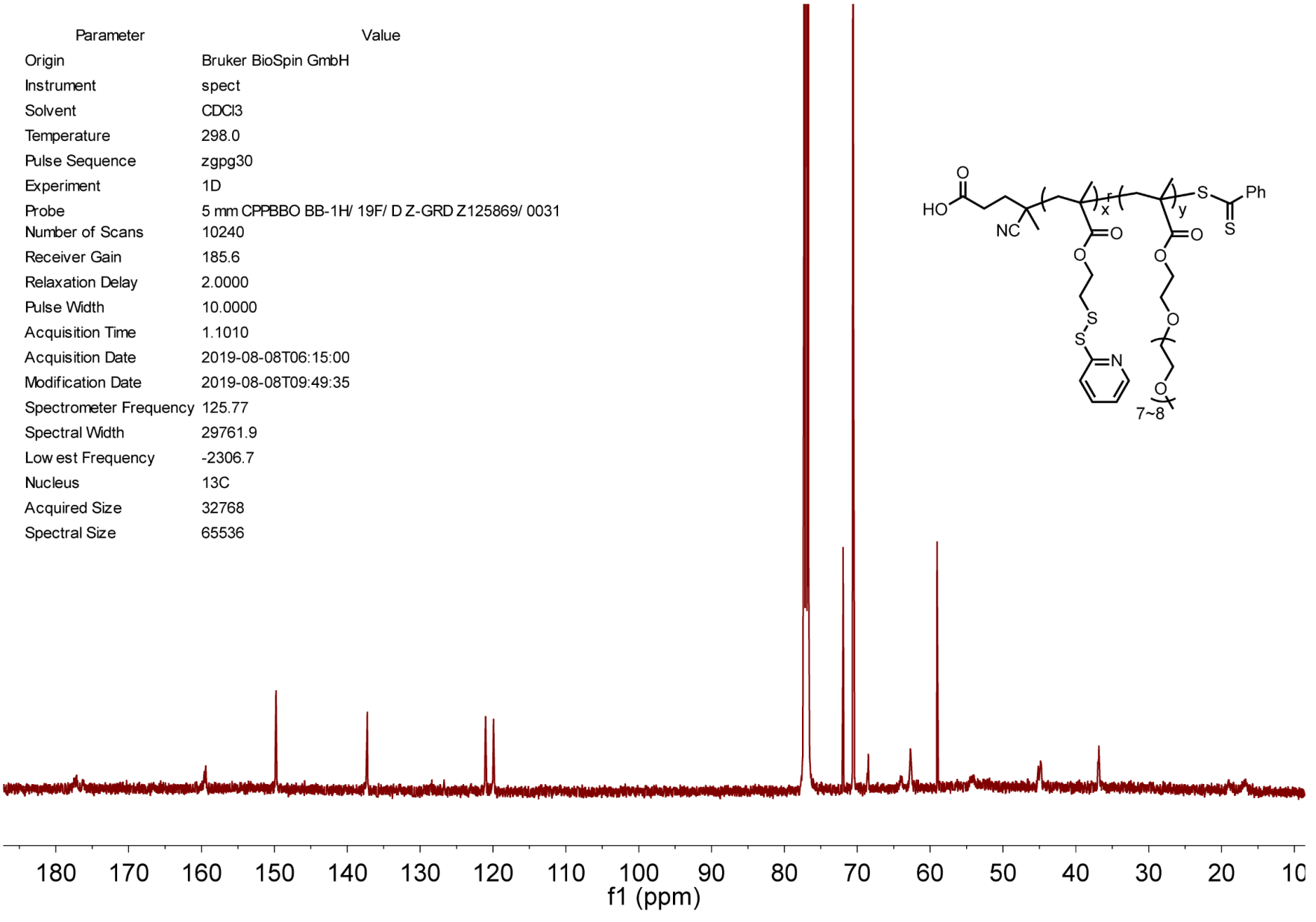

Figure SP8. ${ }^{13} \mathrm{C}$ NMR spectrum $(125 \mathrm{MHz})$ of $\mathbf{R 7 3}$ in $\mathrm{CDCl}_{3}$. 
a)
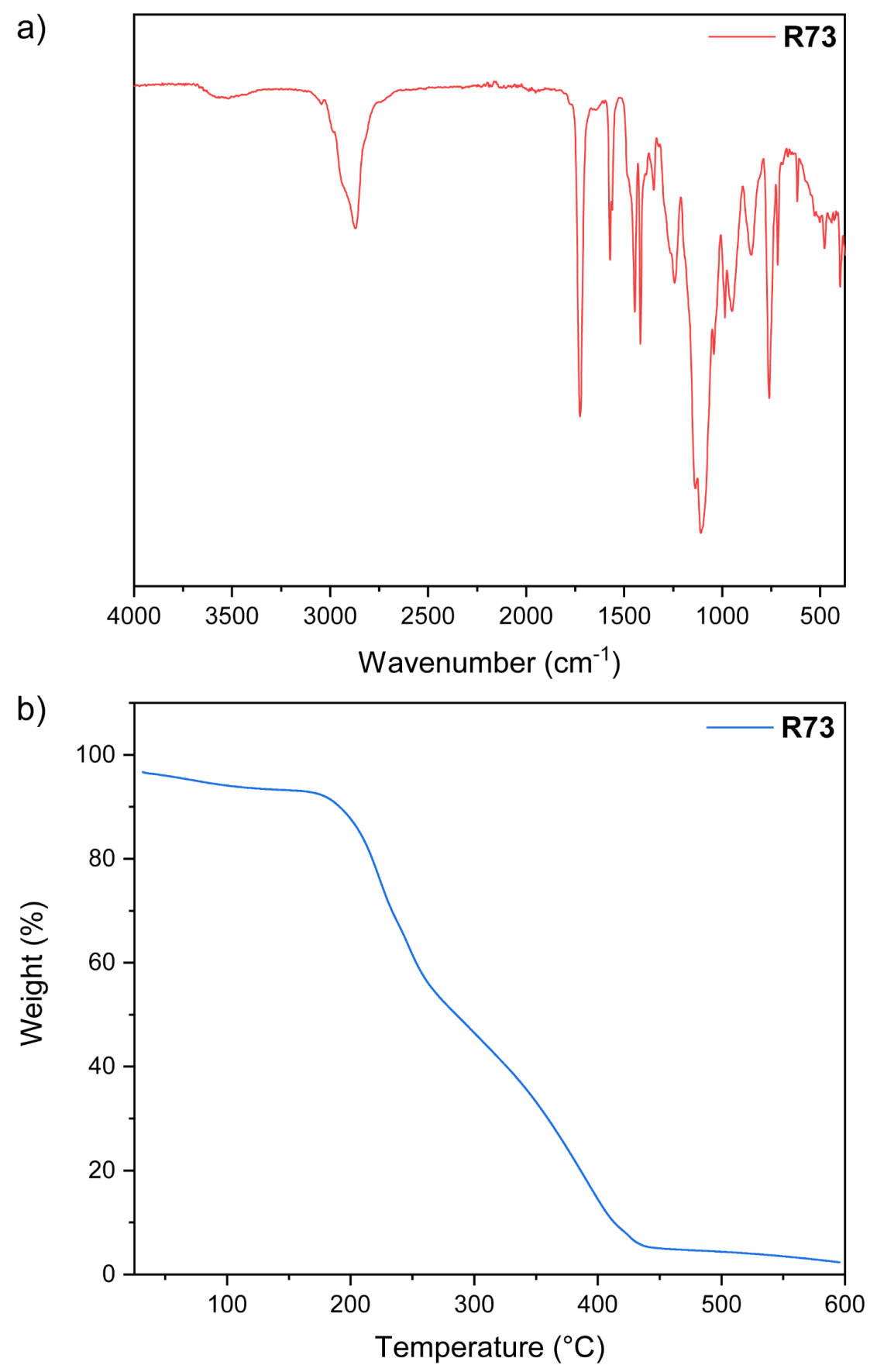

Figure SP9. (a) IR spectrum and (b) TGA curve of R73. 


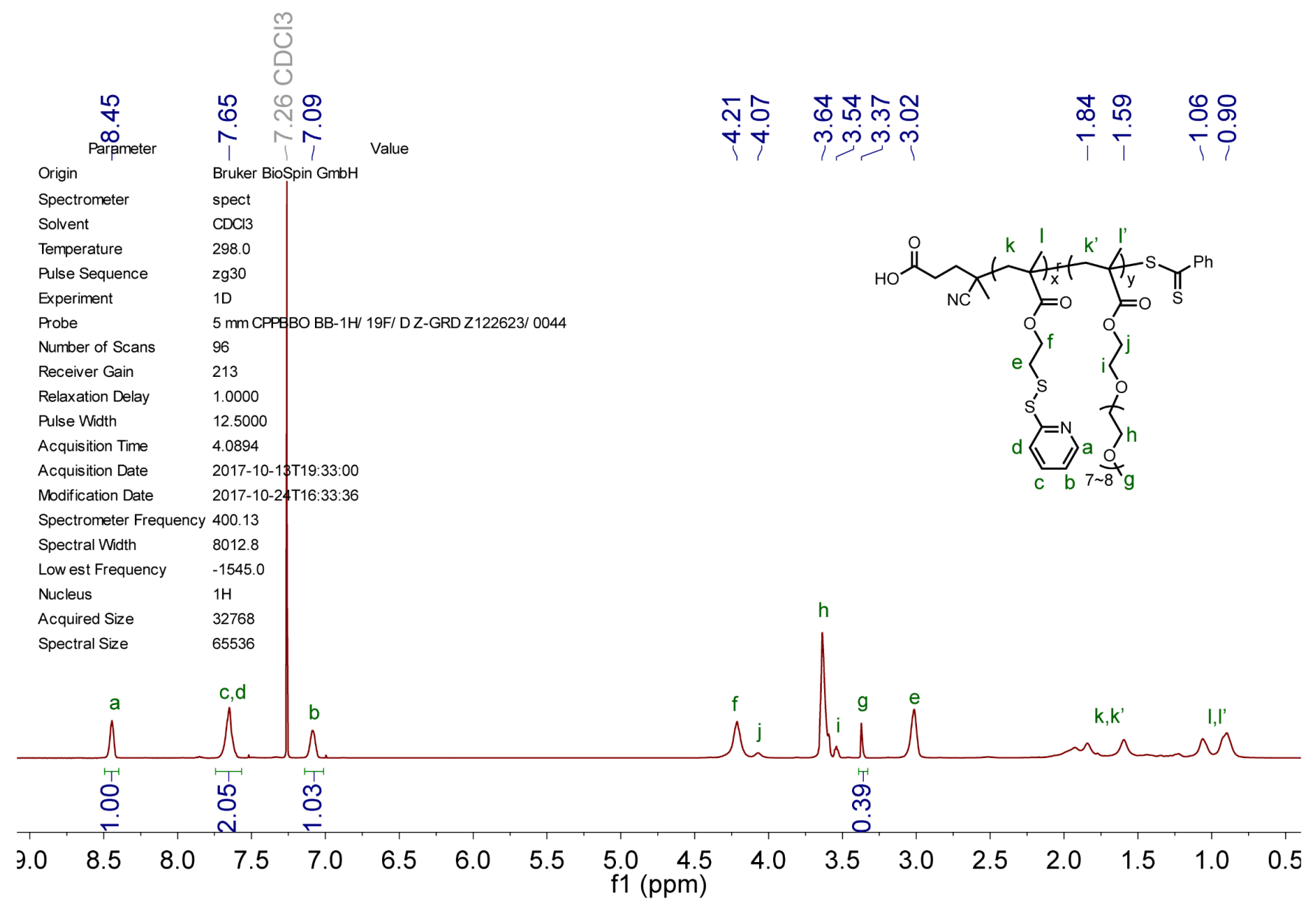

Figure SP10. ${ }^{1} \mathrm{H}$ NMR spectrum $(400 \mathrm{MHz})$ of $\mathbf{R 9 1}$ in $\mathrm{CDCl}_{3}$. 


\begin{tabular}{ll}
\multicolumn{1}{c}{ Parameter } & \multicolumn{2}{c}{ Value } \\
Origin & Bruker BioSpin GmbH \\
Instrument & spect \\
Solvent & CDCl3 \\
Temperature & 298.0 \\
Pulse Sequence & zgpg30 \\
Experiment & $1 \mathrm{D}$ \\
Probe & $5 \mathrm{~mm}$ CPPBBO BB-1H 19F/ D Z-GRD Z125869/ 0031 \\
Number of Scans & 10240 \\
Receiver Gain & 185.6 \\
Relaxation Delay & 2.0000 \\
Pulse Width & 10.0000 \\
Acquisition Time & 1.1010 \\
Acquisition Date & $2019-08-09$ T05:18:00 \\
Modification Date & $2019-08-09 T 13: 45: 31$ \\
Spectrometer Frequency & 125.77 \\
Spectral Width & 29761.9 \\
Low est Frequency & -2306.9 \\
Nucleus & $13 \mathrm{C}$ \\
Acquired Size & 32768 \\
Spectral Size & 65536 \\
&
\end{tabular}
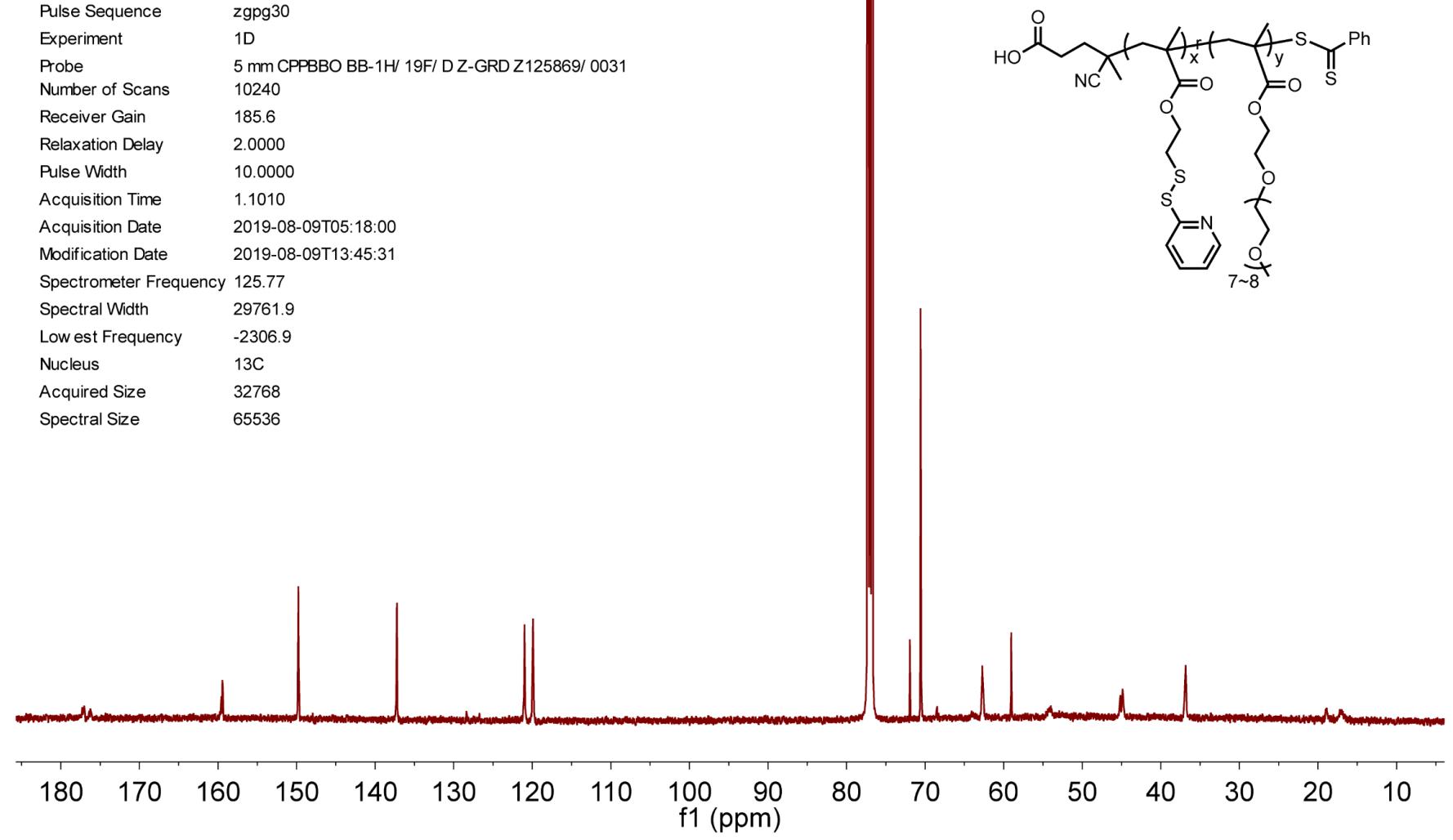

Figure SP11. ${ }^{13} \mathrm{C}$ NMR spectrum $(125 \mathrm{MHz})$ of $\mathbf{R} 91$ in $\mathrm{CDCl}_{3}$. 
a)
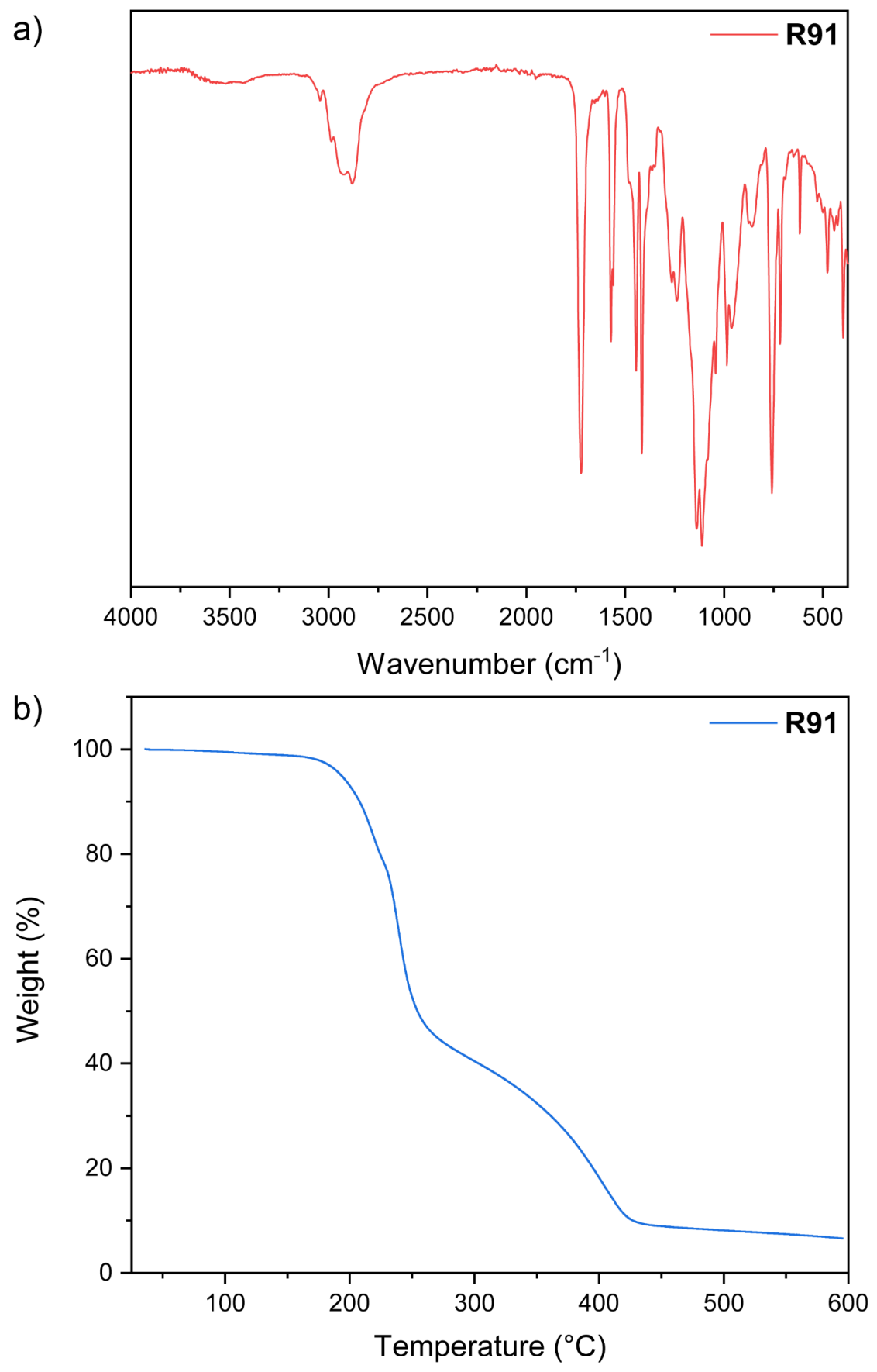

Figure SP12. (a) IR spectrum and (b) TGA curve of R91. 


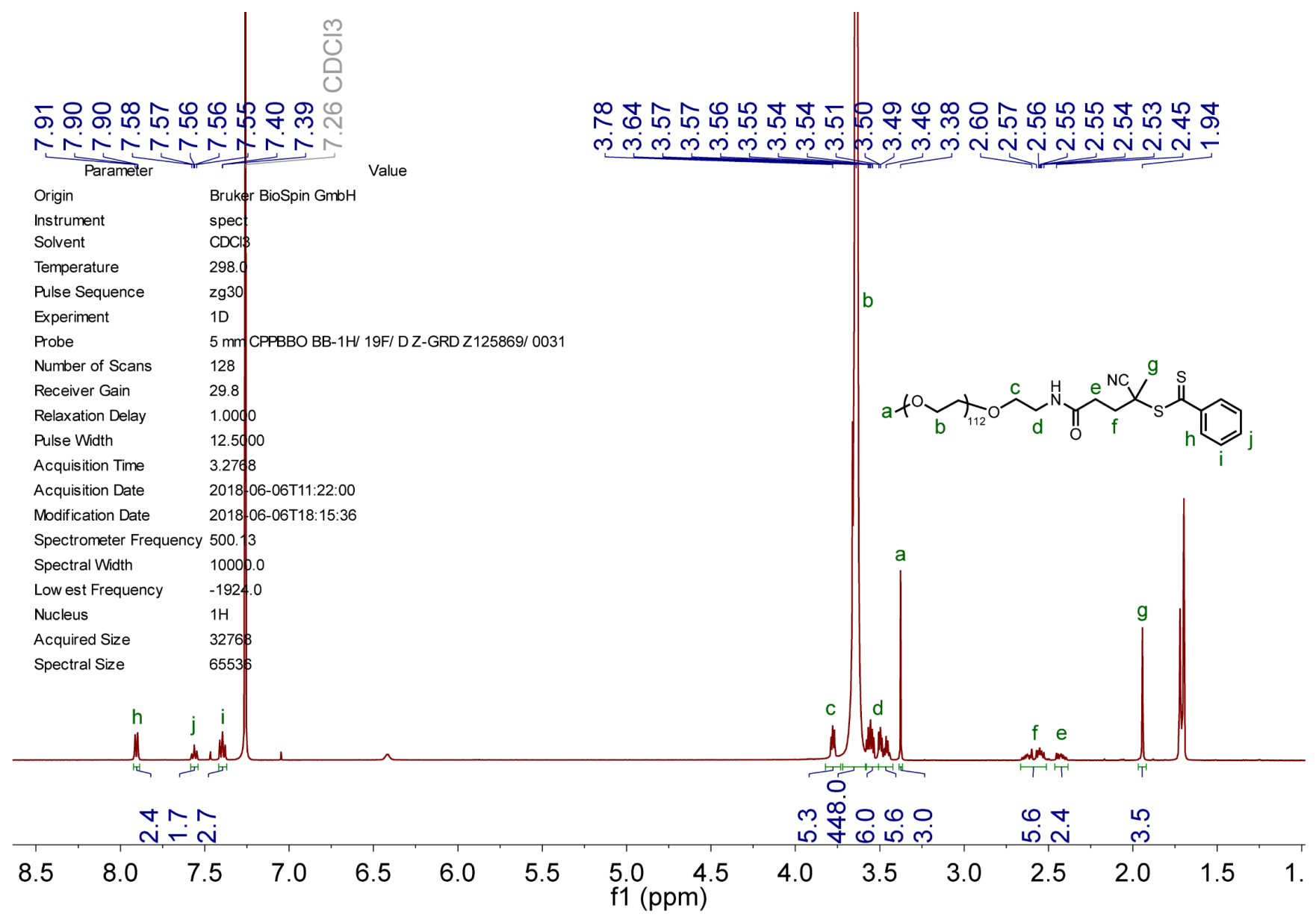

Figure SP13. ${ }^{1} \mathrm{H}$ NMR spectrum $(500 \mathrm{MHz})$ of macro-CTA in $\mathrm{CDCl}_{3}$. 


\begin{tabular}{ll}
\multicolumn{1}{c}{ Parameter } & \\
Origin & Bruker BioSpin GmbH \\
Instrument & spect \\
Solvent & CDCl3 \\
Temperature & 298.0 \\
Pulse Sequence & zgpg30 \\
Experiment & $1 \mathrm{D}$ \\
Probe & $5 \mathrm{~mm}$ CPPBBO BB-1H/ 19F/ D Z-GRD Z125869/ 0031 \\
Number of Scans & 1024 \\
Receiver Gain & 185.6 \\
Relaxation Delay & 2.0000 \\
Pulse Width & 10.0000 \\
Acquisition Time & 1.1010 \\
Acquisition Date & $2018-06-06 \mathrm{~T} 12: 24: 00$ \\
Modification Date & $2018-06-06 \mathrm{~T} 18: 15: 33$ \\
Spectrometer Frequency & 125.77 \\
Spectral Width & 29761.9 \\
Low est Frequency & -2305.9 \\
Nucleus & $13 \mathrm{C}$ \\
Acquired Size & 32768 \\
Spectral Size & 65536 \\
&
\end{tabular}
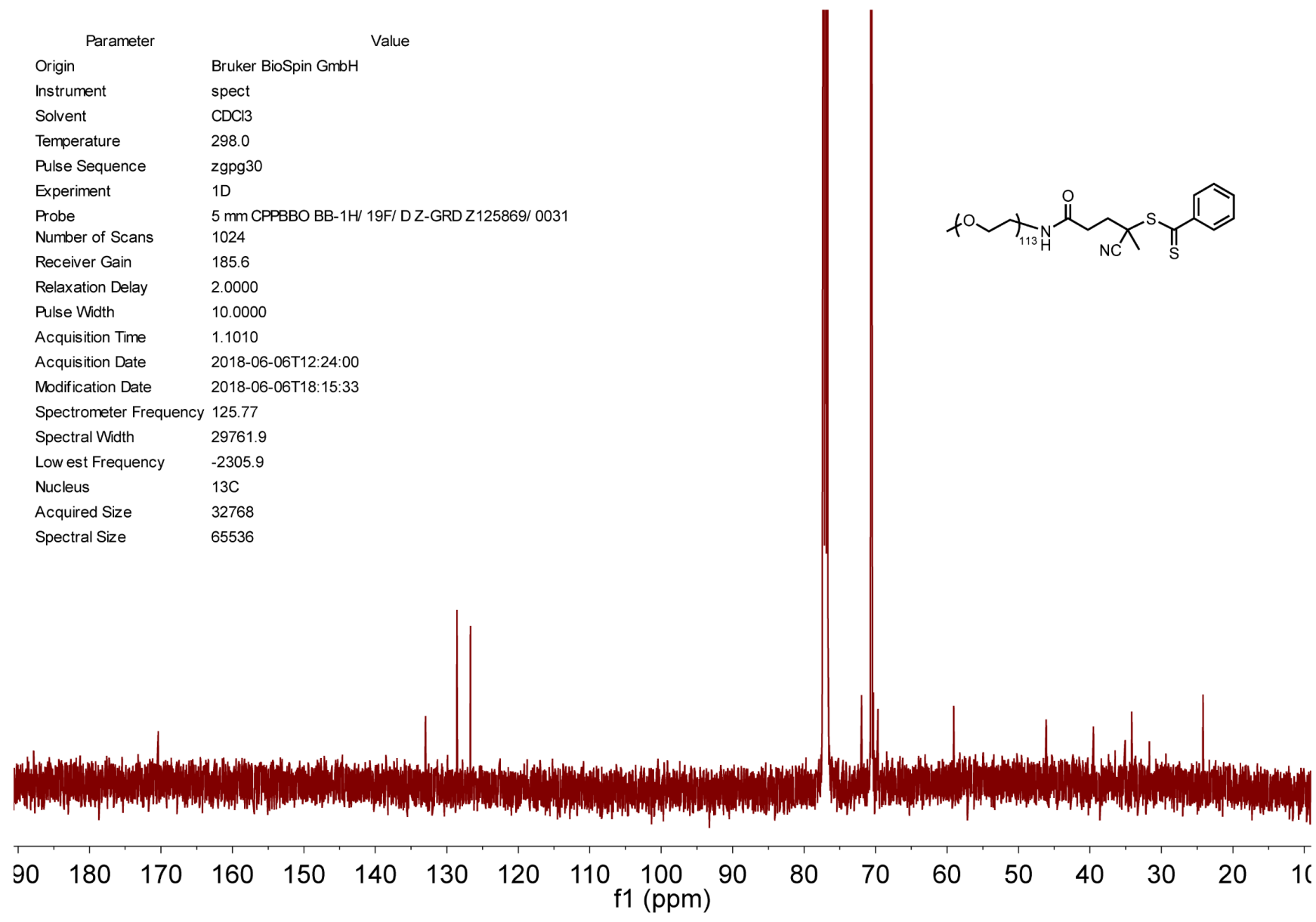

Figure SP14. ${ }^{13} \mathrm{C}$ NMR spectrum $(125 \mathrm{MHz})$ of macro-CTA in $\mathrm{CDCl}_{3}$. 


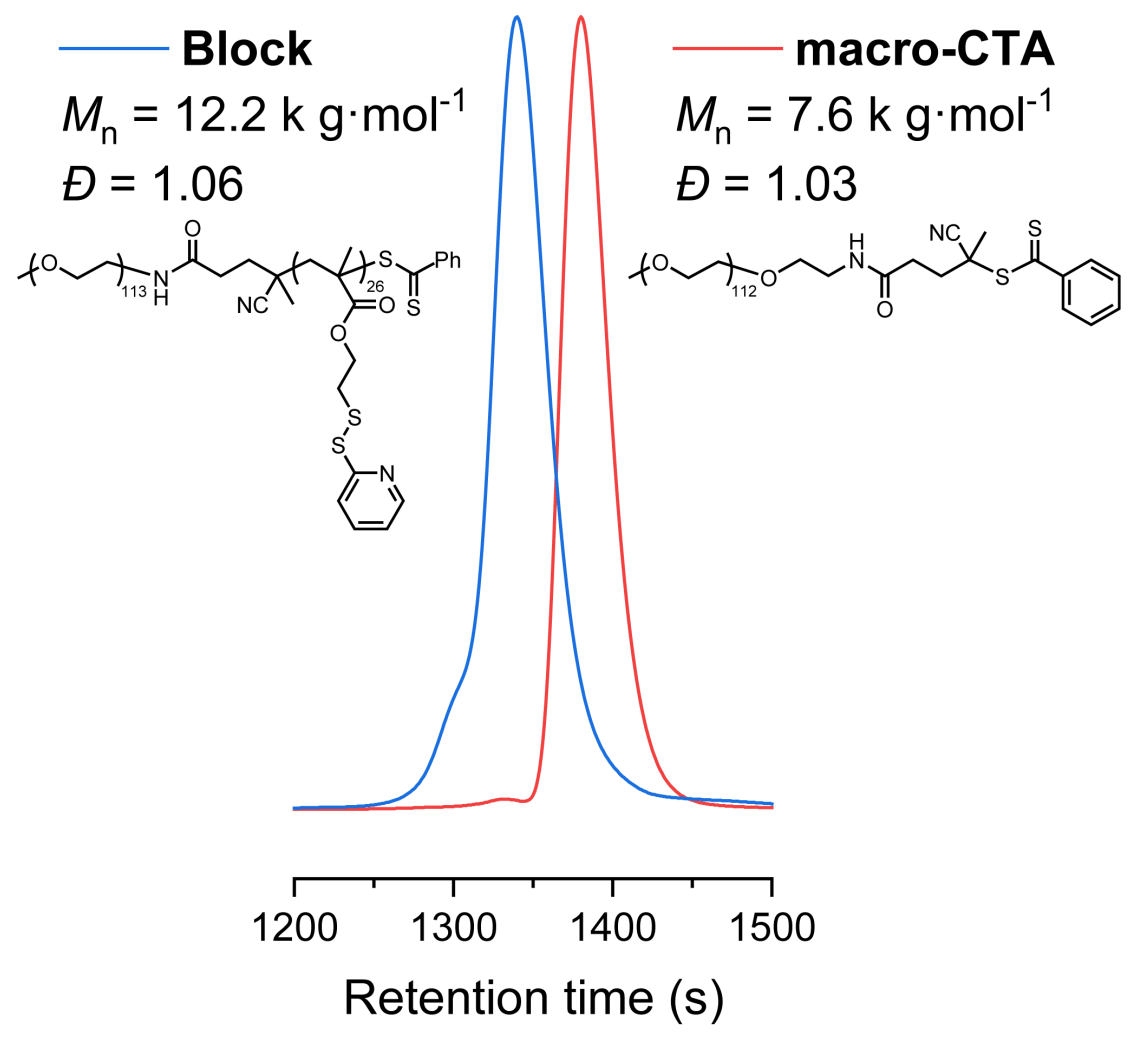

Figure SP15. Gel permeation chromatography (GPC) curves of macro-CTA and B73. 


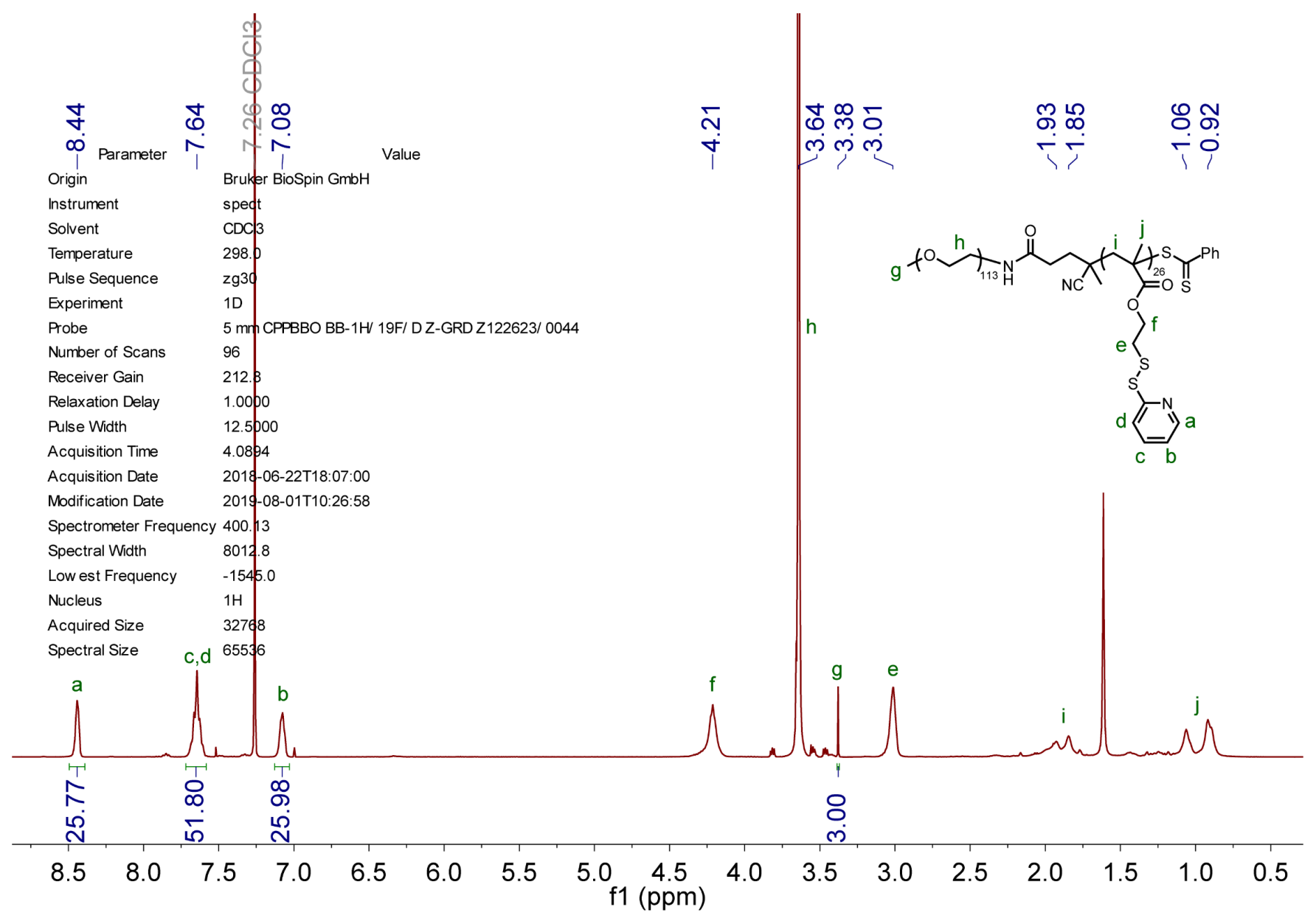

Figure SP16. ${ }^{1} \mathrm{H}$ NMR spectrum $(400 \mathrm{MHz})$ of $\mathbf{B} 73$ in $\mathrm{CDCl}_{3}$. 


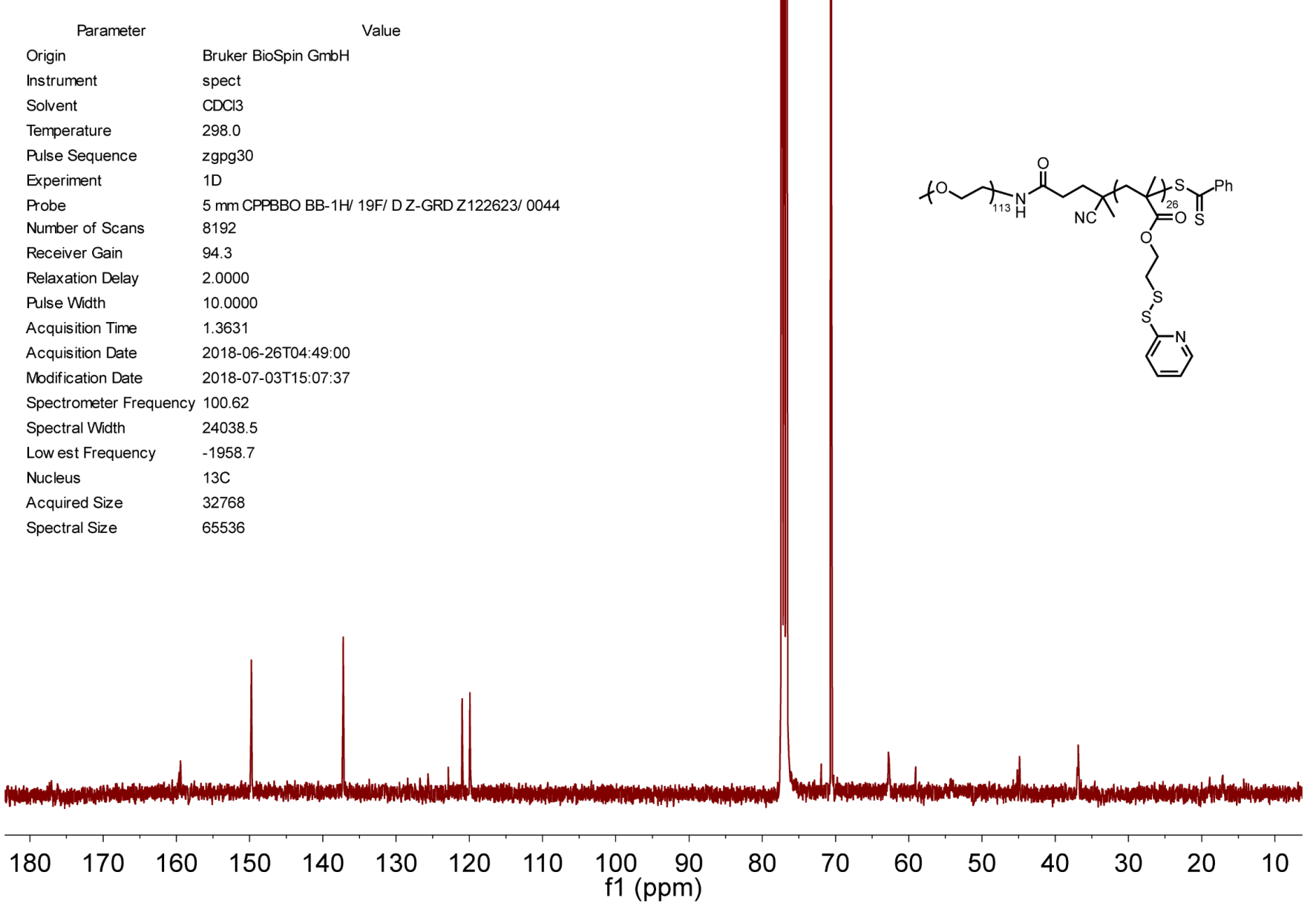

Figure SP17. ${ }^{13} \mathrm{C}$ NMR spectrum $(100 \mathrm{MHz})$ of $\mathbf{B} 73$ in $\mathrm{CDCl}_{3}$. 
a)

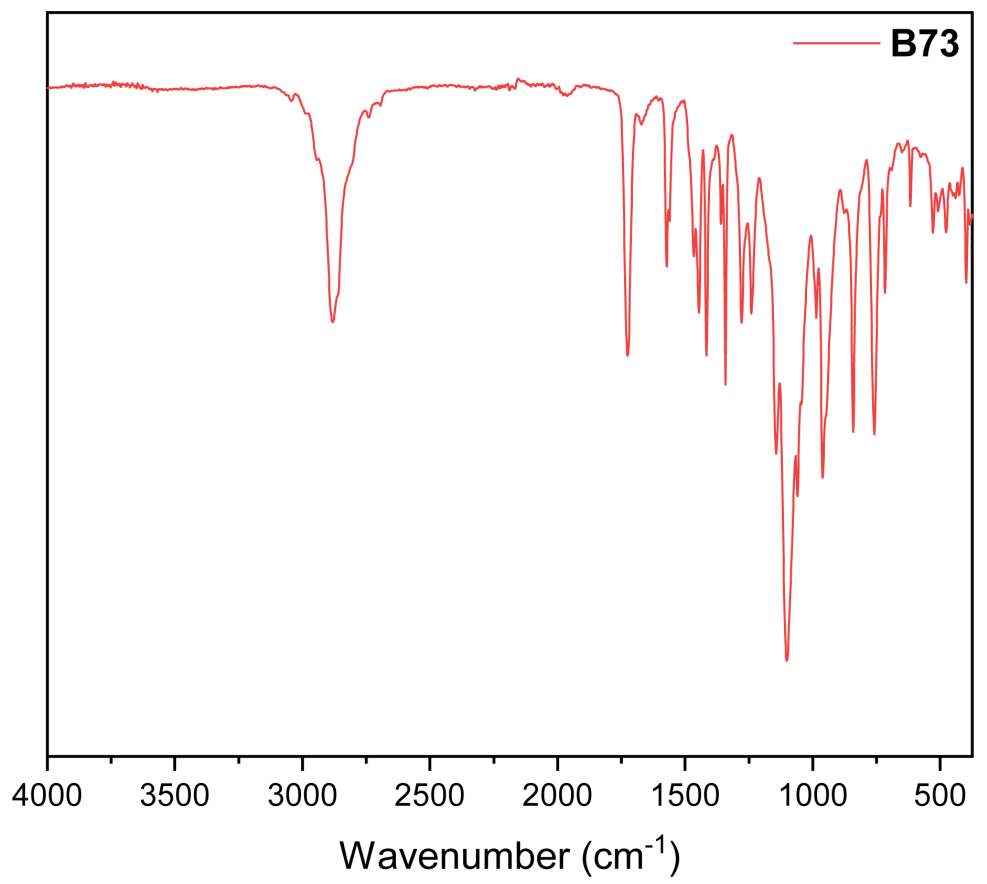

b)

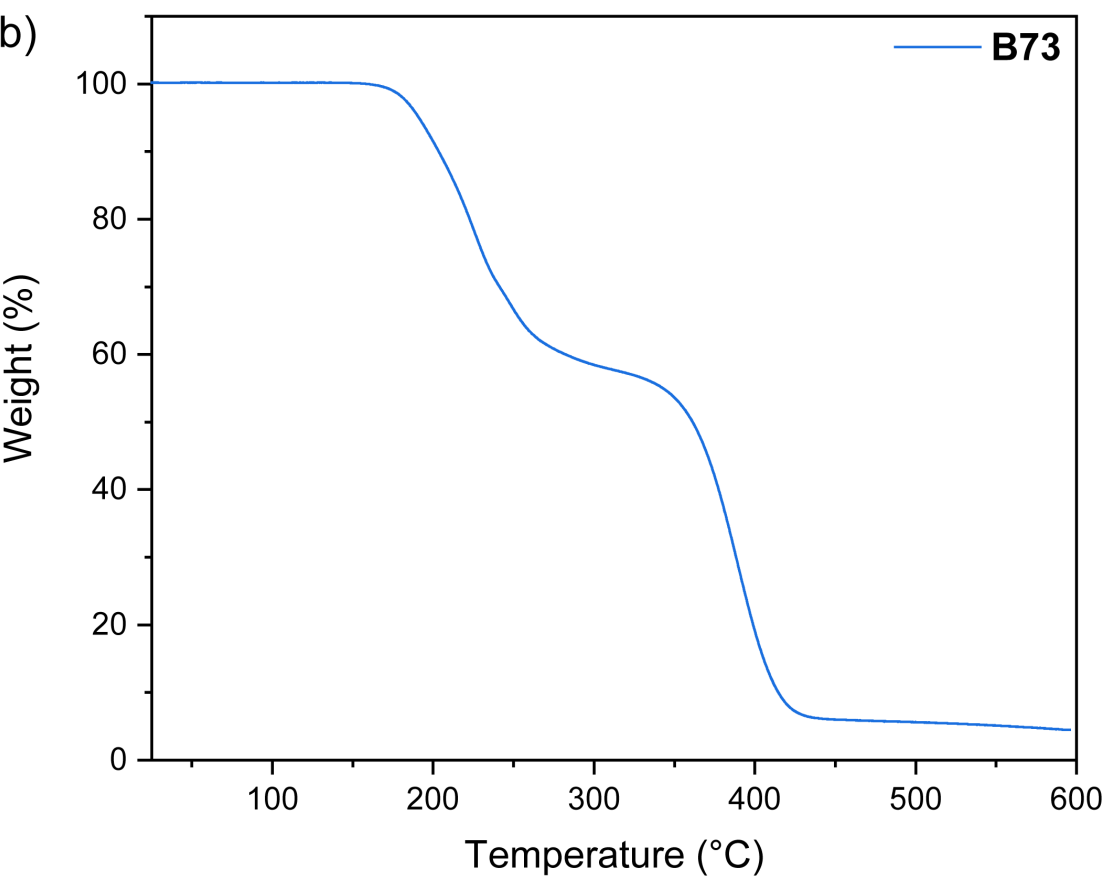

Figure SP18. (a) IR spectrum and (b) TGA curve of B73. 


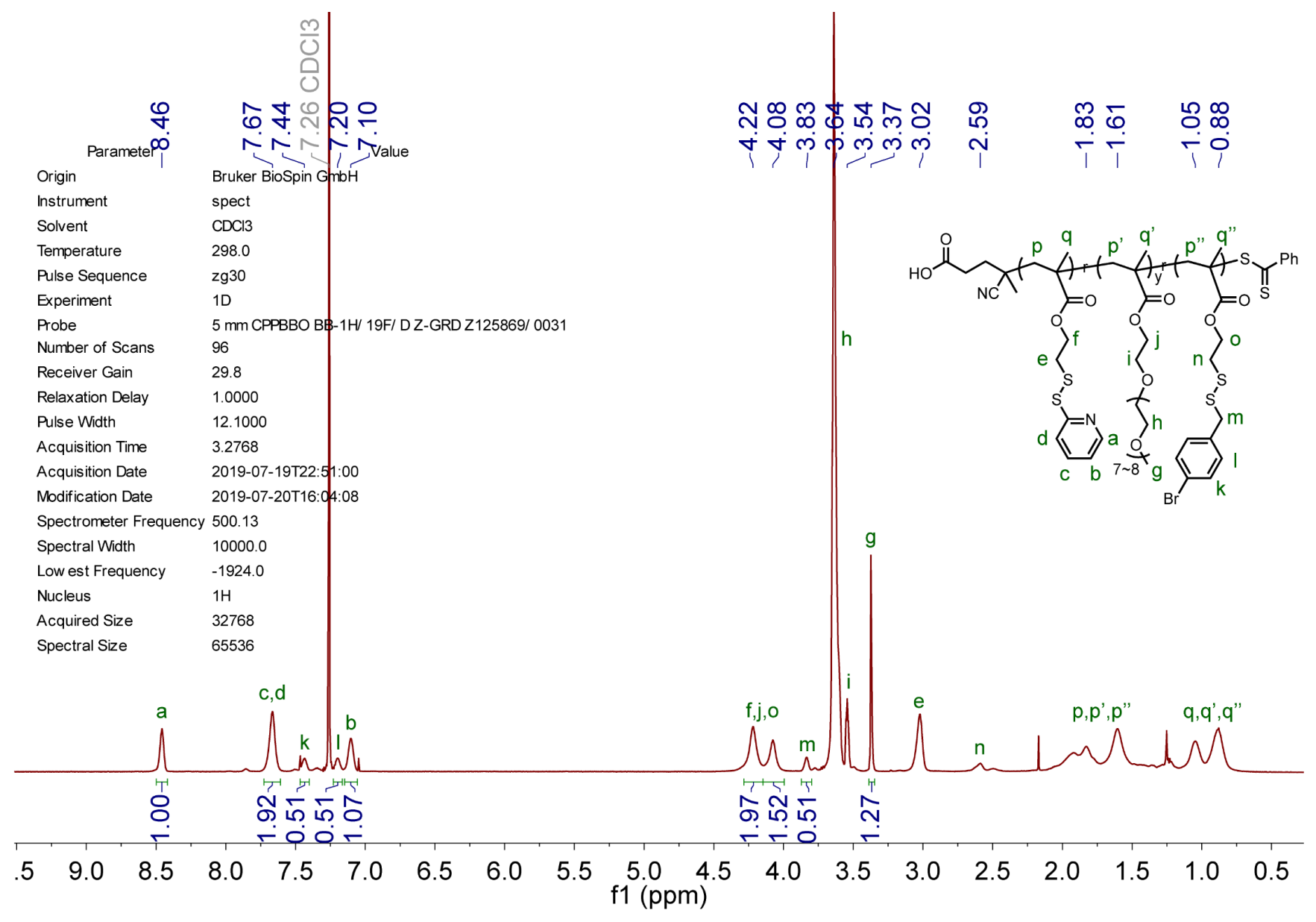

Figure SP19. ${ }^{1} \mathrm{H}$ NMR spectrum $(500 \mathrm{MHz})$ of $\mathbf{R 7 3}-\mathrm{Br}$ in $\mathrm{CDCl}_{3}$. 

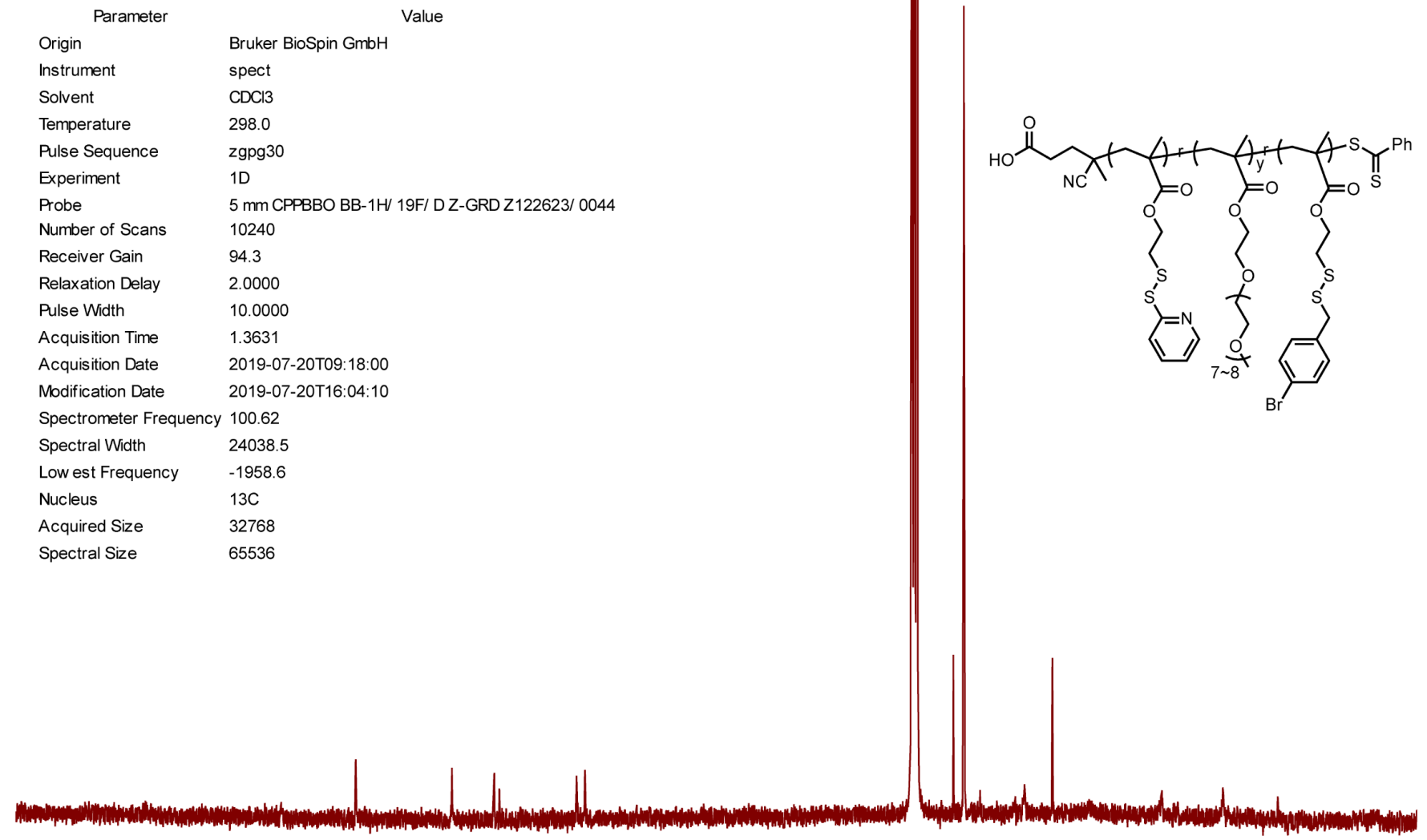

$\begin{array}{llllllllllllllllll}190 & 180 & 170 & 160 & 150 & 140 & 130 & 120 & \begin{array}{c}110 \\ \mathrm{f} 1(\mathrm{ppm})\end{array} & 90 & 80 & 70 & 60 & 50 & 40 & 30 & 20\end{array}$

Figure SP20. ${ }^{13} \mathrm{C}$ NMR spectrum $(100 \mathrm{MHz})$ of $\mathbf{R 7 3}-\mathbf{B r}$ in $\mathrm{CDCl}_{3}$. 
a)

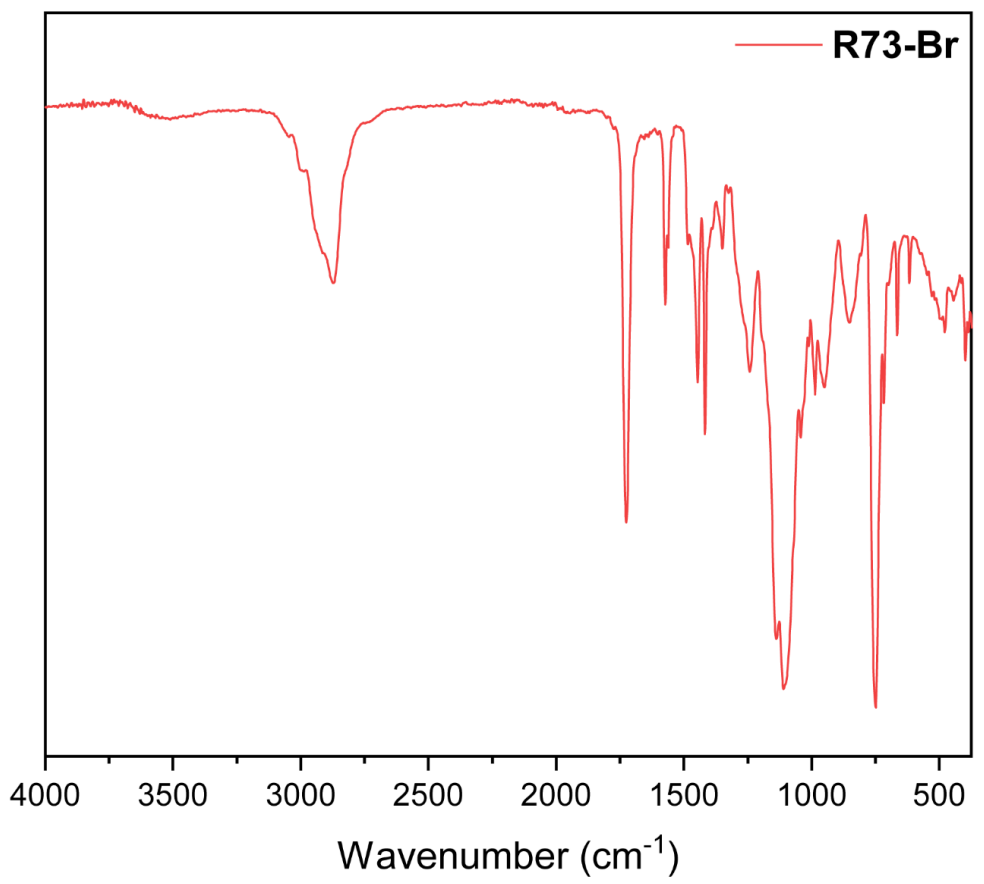

b)

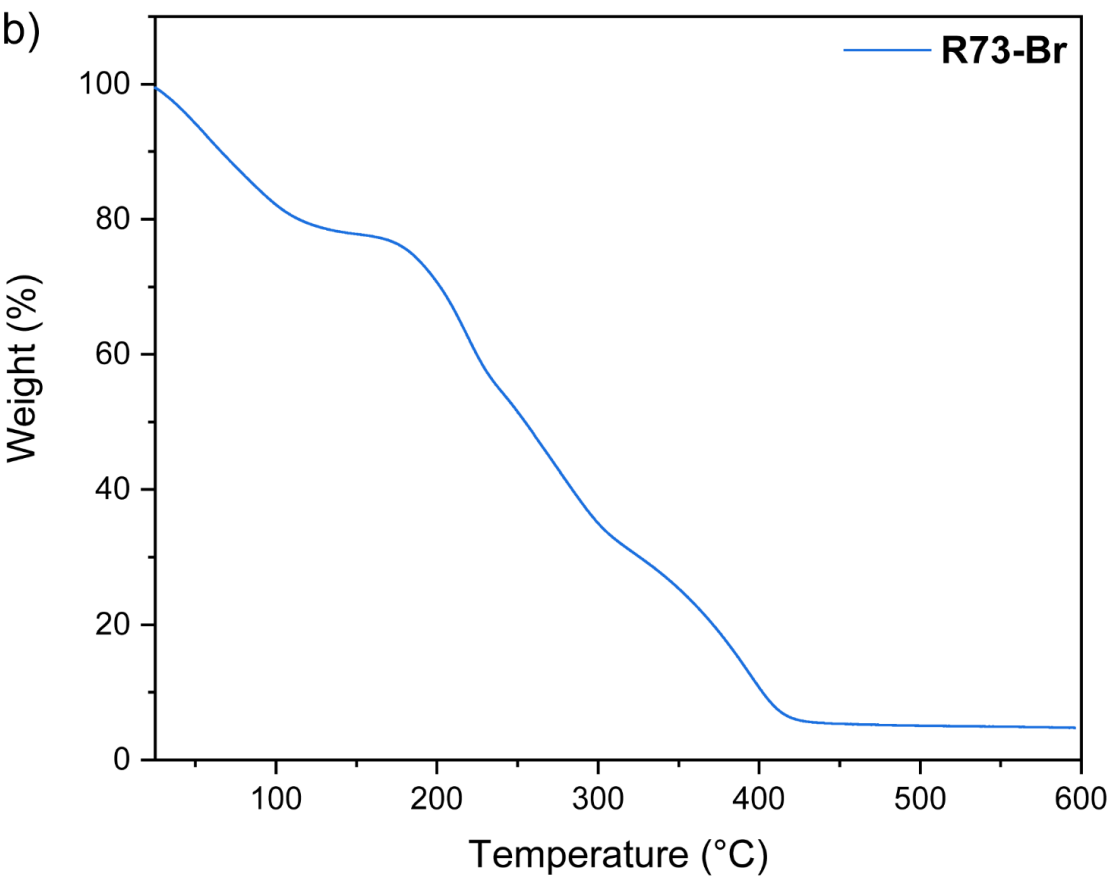

Figure SP21. (a) IR spectrum and (b) TGA curve of R73-Br. 


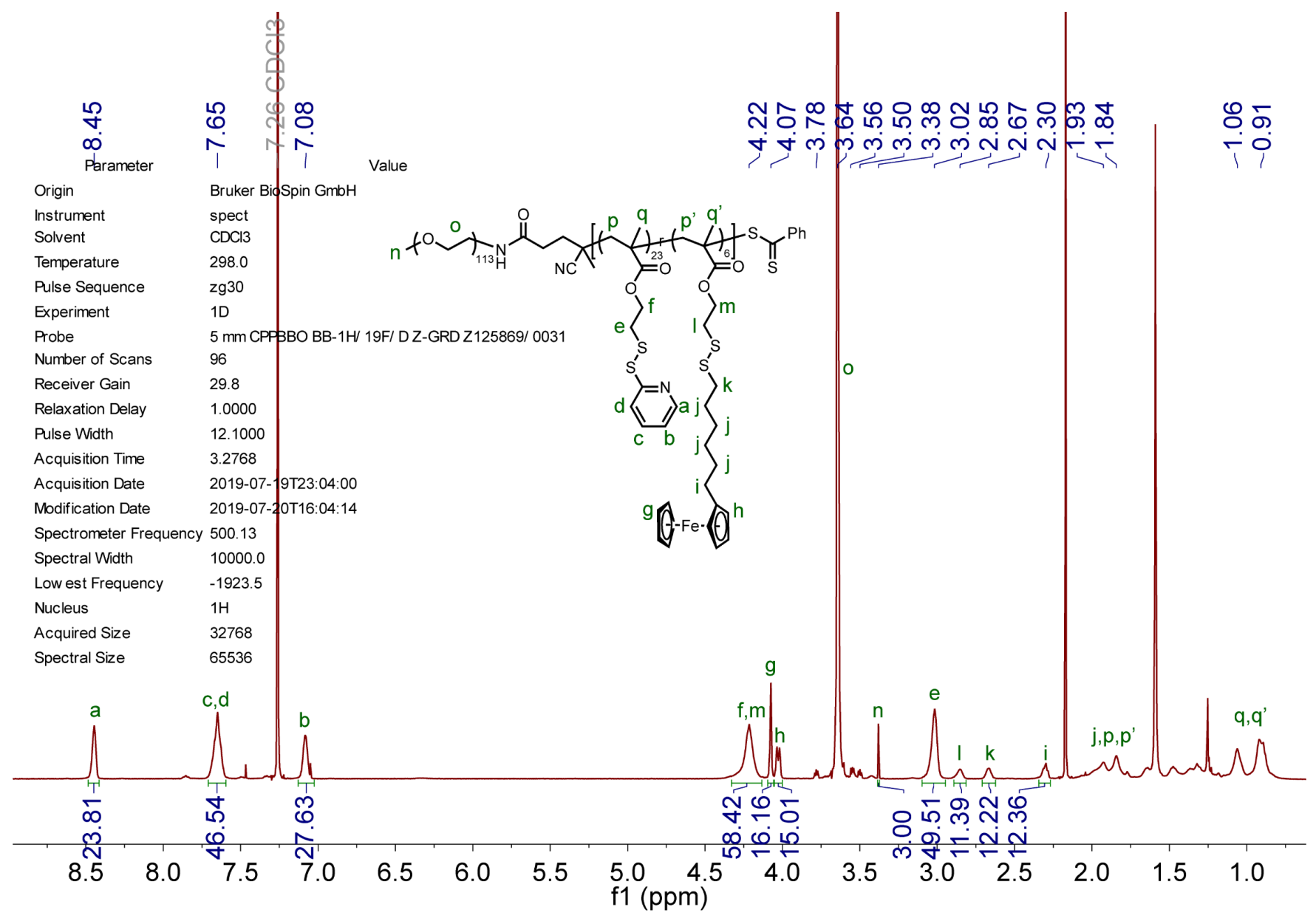

Figure SP22. ${ }^{1} \mathrm{H}$ NMR spectrum $(500 \mathrm{MHz})$ of $\mathbf{B} 73-\mathrm{Fe}$ in $\mathrm{CDCl}_{3}$. 


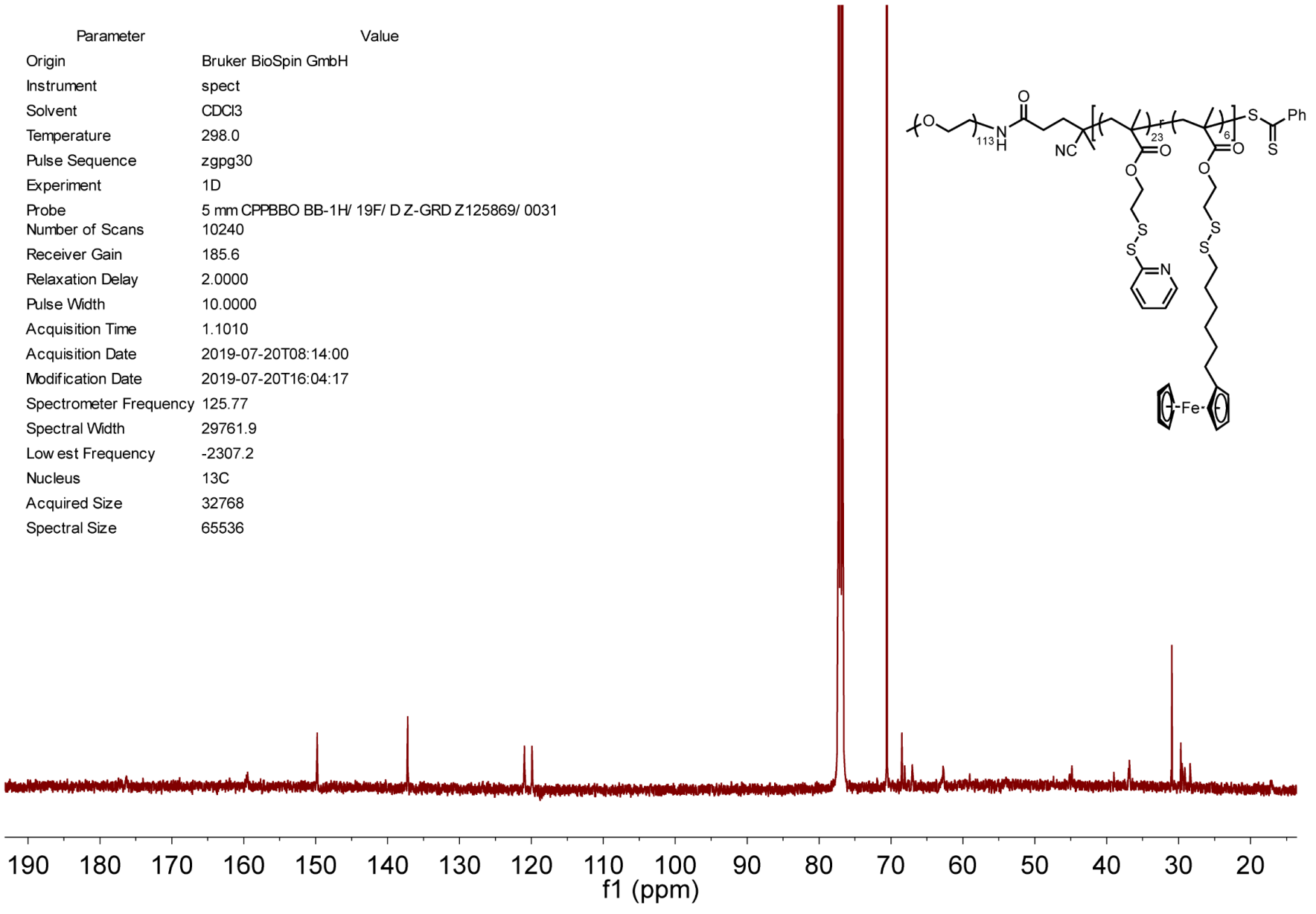

Figure SP23. ${ }^{13} \mathrm{C}$ NMR spectrum $(125 \mathrm{MHz})$ of $\mathrm{B} 73-\mathrm{Fe}$ in $\mathrm{CDCl}_{3}$. 
a)

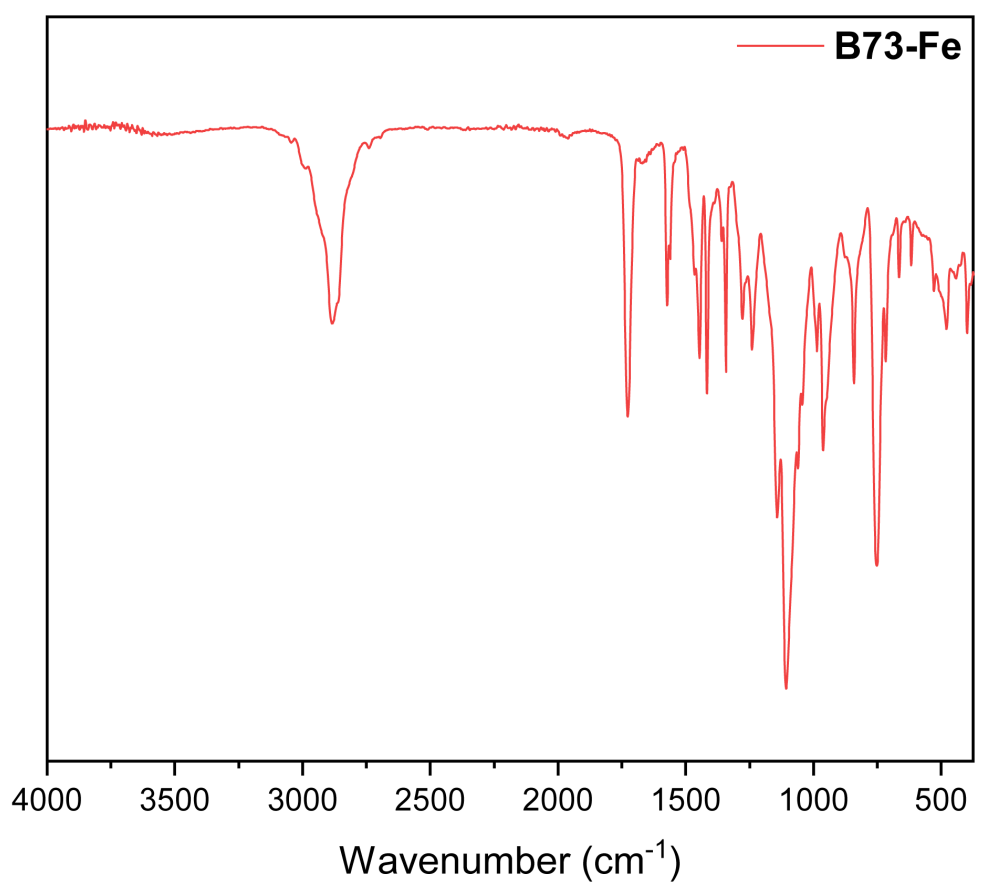

b)

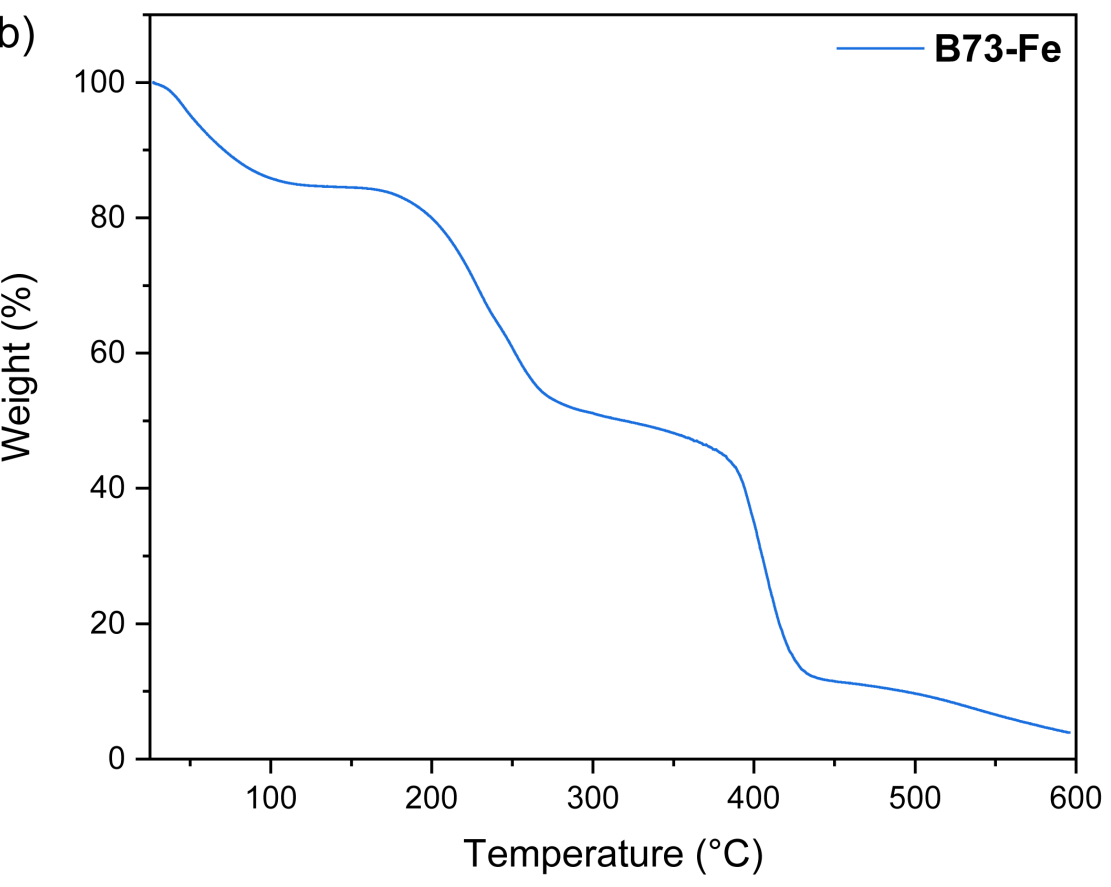

Figure SP24. (a) IR spectrum and (b) TGA curve of B73-Fe. 


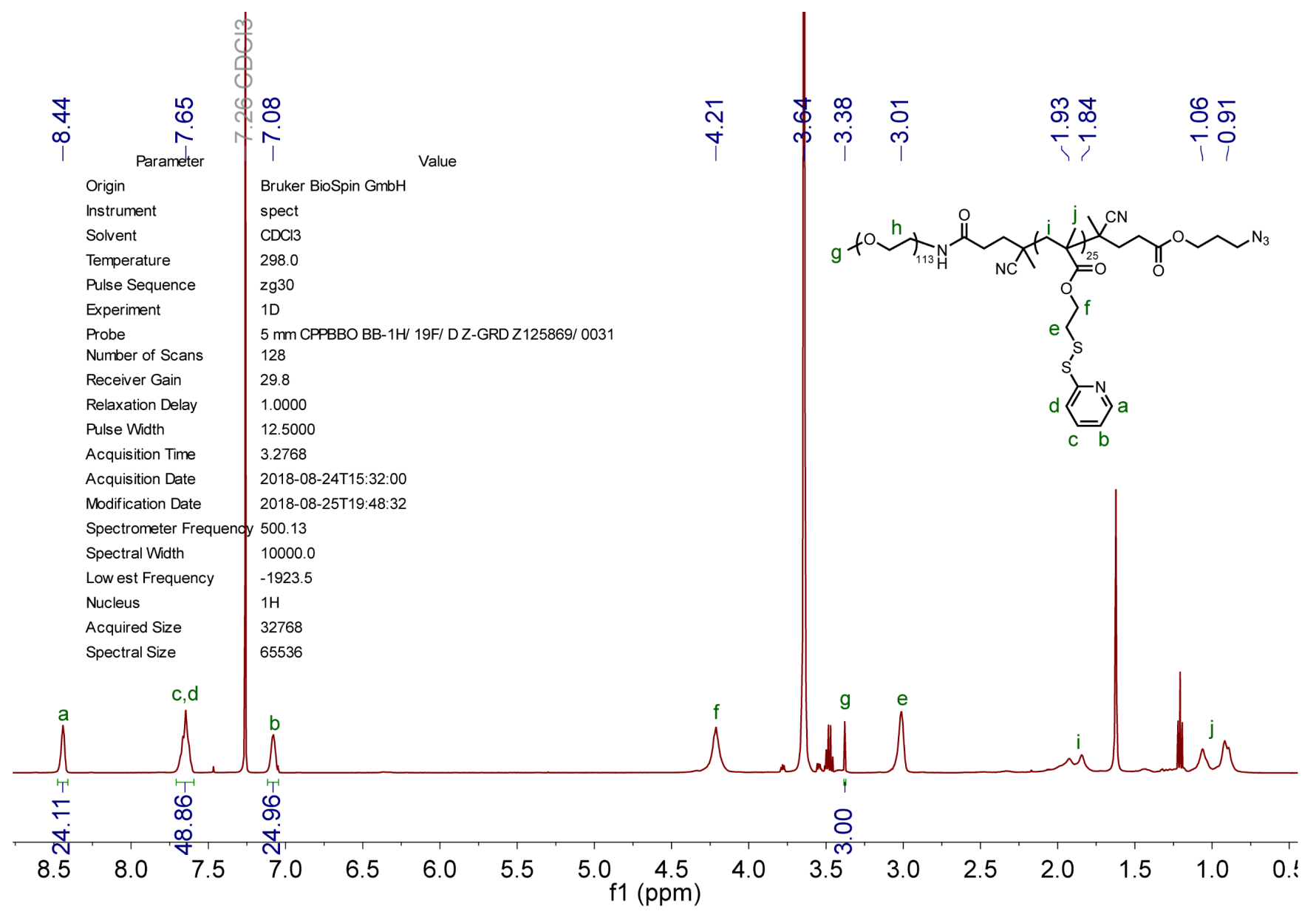

Figure SP25. ${ }^{1} \mathrm{H}$ NMR spectrum $(500 \mathrm{MHz})$ of $\mathbf{B} 73-\mathbf{N}_{\mathbf{3}}$ in $\mathrm{CDCl}_{3}$. 


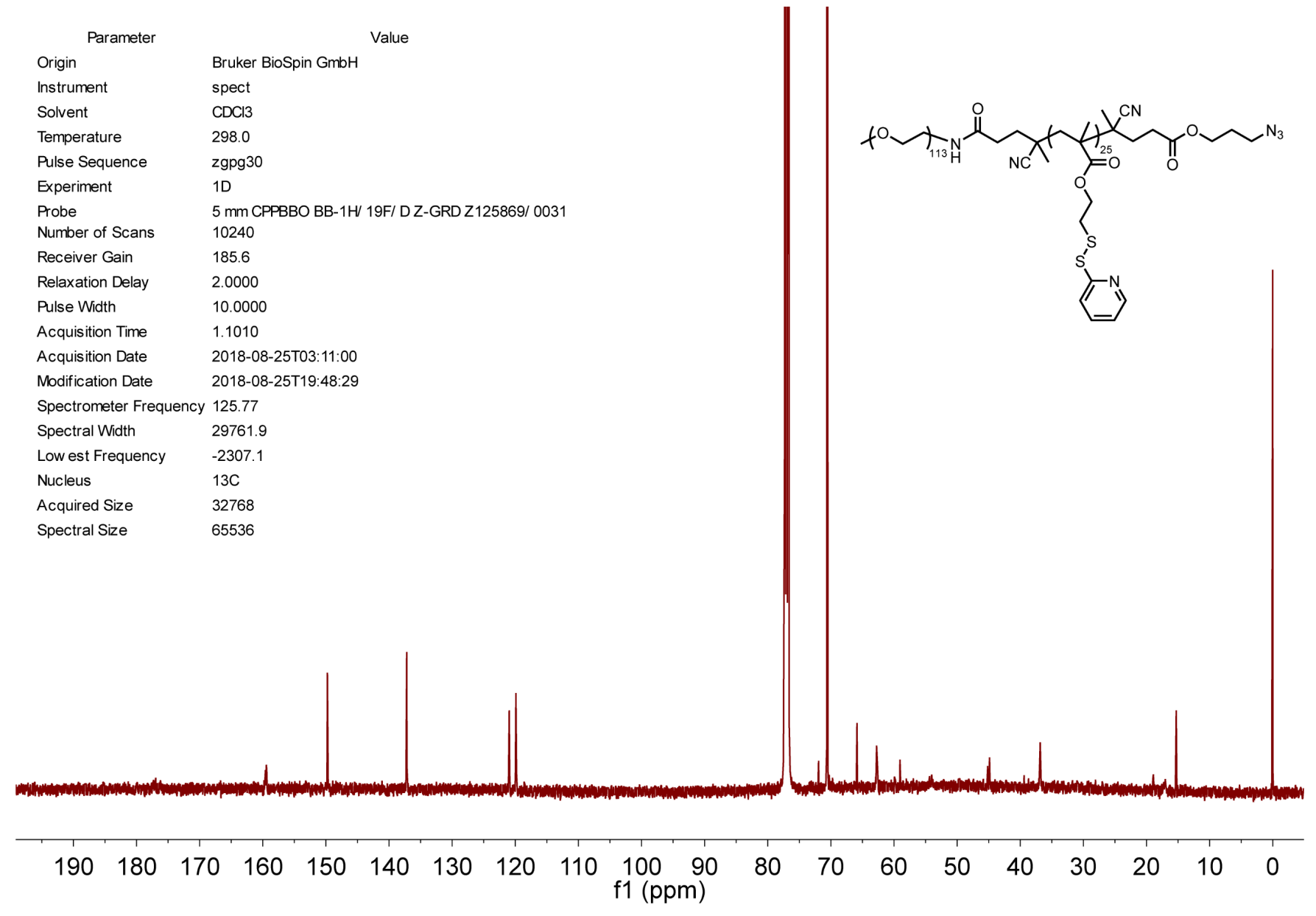

Figure SP26. ${ }^{13} \mathrm{C}$ NMR spectrum $(125 \mathrm{MHz})$ of $\mathbf{B} 73-\mathbf{N}_{\mathbf{3}}$ in $\mathrm{CDCl}_{3}$. 


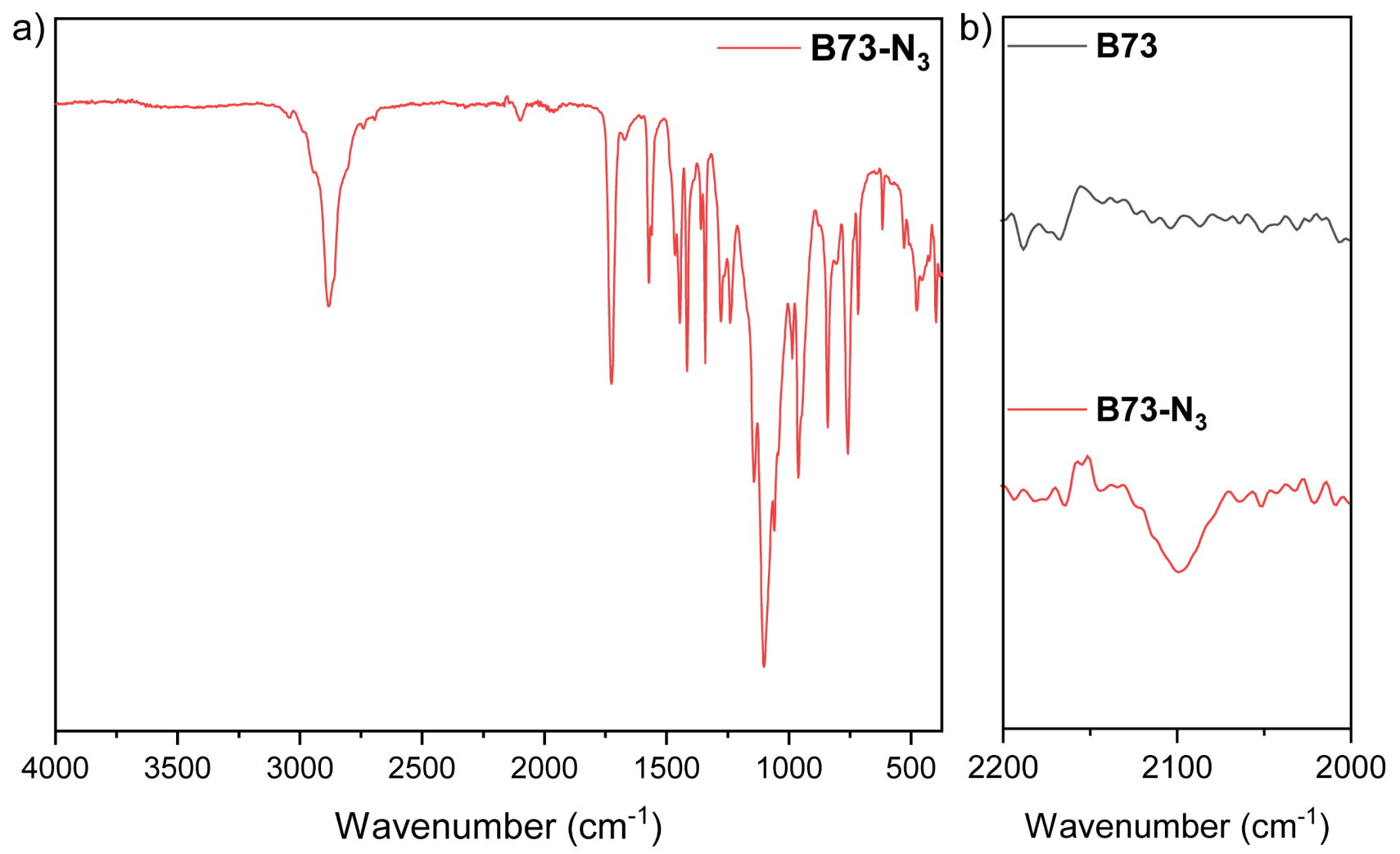

Figure SP27. FTIR spectra of (a) $\mathbf{B} 73-\mathbf{N}_{3}$ and (b) the comparison between $\mathbf{B} 73$ and $\mathbf{B} 73-\mathbf{N}_{3}$, demonstrating the appearance of the azide group $\left(2100 \mathrm{~cm}^{-1}\right)$ in $\mathbf{B} 73-\mathbf{N}_{\mathbf{3}}$.

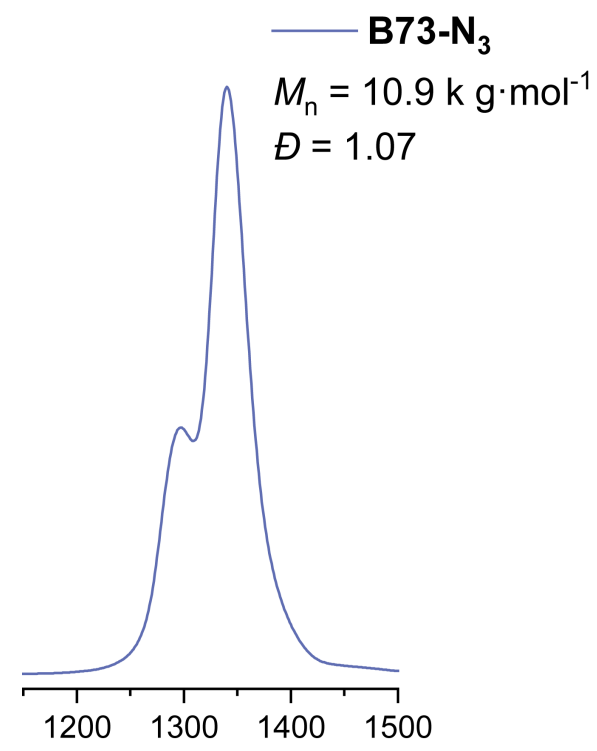

Retention time (s)

Figure SP28. Gel permeation chromatography (GPC) curve of B73-N 3 . 


\section{References}

1. Delage, B.; Briou, B.; Brossier, T.; Catrouillet, S.; Robin, J.-J.; Lapinte, V., Polyoxazoline associated with cardanol for bio-based linear alkyl benzene surfactants. Polym. Int. 2019, 68 (4), 755-763.

2. Warren, N. J.; Mykhaylyk, O. O.; Mahmood, D.; Ryan, A. J.; Armes, S. P., RAFT Aqueous Dispersion Polymerization Yields Poly(ethylene glycol)-Based Diblock Copolymer Nano-Objects with Predictable Single Phase Morphologies. J. Am. Chem. Soc. 2014, 136 (3), 1023-1033.

3. Jiang, Z.; He, H.; Liu, H.; Thayumanavan, S., Cellular Uptake Evaluation of Amphiphilic Polymer Assemblies: Importance of Interplay between Pharmacological and Genetic Approaches. Biomacromolecules 2019, 20 (12), 4407-4418.

4. Chong, Y. K.; Moad, G.; Rizzardo, E.; Thang, S. H., Thiocarbonylthio End Group Removal from RAFT-Synthesized Polymers by Radical-Induced Reduction. Macromolecules 2007, 40 (13), 44464455. 